\title{
Copper-Catalyzed meta-Selective Arylation of Phenol Derivatives: An Easy Access to $m$-Aryl Phenols
}

\author{
Manikantha Maraswami, ${ }^{\mathrm{c}}$ Hajime Hirao ${ }^{\mathrm{d}, \mathrm{e} *}$ and Teck-Peng Loh ${ }^{\mathrm{a}, \mathrm{b}, \mathrm{c} *}$
}

anstitute of Advanced Synthesis (IAS), School of Chemistry and Chemical Engineering, Northwestern Polytechnical University (NPU), Xi'an 710072, China; ' Yangtze River Delta Research Institute of NPU, Taicang Jiangsu, 215400, China.

'Division of Chemistry and Biological Chemistry, School of Physical and Mathematical Sciences, Nanyang Technological University, Singapore 637371.

dSchool of Life and Health Sciences, The Chinese University of Hong Kong, Shenzhen, Longgang District, Shenzhen, Guangdong Province 518172, China.

eWarshel Institute for Computational Biology, The Chinese University of Hong Kong, Shenzhen, Longgang District, Shenzhen, Guangdong Province 518172, China.

\section{teckpeng@ntu.edu.sg \\ hirao@cuhk.edu.cn}

\section{Supporting Information}

I. General methods

II. Procedure and characterization data

S2-S18

III. Gram scale synthesis

S18

IV. Diversification of $\mathbf{3 r}$

S19-S20

V. DFT Calculations

S20-S32

VI. References

S33-S34

VII. ${ }^{1} \mathrm{H}$ and ${ }^{13} \mathrm{C}$ NMR spectra of Compounds

S35-S75 


\section{General Methods}

$\mathrm{Cu}(\mathrm{OTf})_{2}, \mathrm{Cu}(\mathrm{OAc})_{2}, \mathrm{CuI}, \mathrm{CuBr}_{2}$ and copper powder were purchased from commercial suppliers and used as received unless otherwise noted. All reactions were carried out under air unless otherwise stated. Commercial solvents and reagents were used without further purification. Reactions were monitored through thin layer chromatography [Merck 60 F254 precoated silica gel plate $(0.2 \mathrm{~mm}$ thickness $)]$. Subsequent to elution, spots were visualized using UV radiation $(254 \mathrm{~nm})$ on Spectroline Model ENF-24061/F $254 \mathrm{~nm}$. Further visualization was possible using basic solution of potassium permanganate or acidic solution of ceric molybdate as stain, followed by heating on a hot plate. Flash chromatography was performed using Merck silica gel 60 with distilled solvents. HRMS spectra were recorded on a Waters Q-Tof Permier Spectrometer. ${ }^{1} \mathrm{H}$ NMR and ${ }^{13} \mathrm{C}$ NMR spectra were recorded using Bruker Avance $400 \mathrm{MHz}$ spectrometers. Chemical shifts for ${ }^{1} \mathrm{H}$ NMR spectra are reported as $\delta$ in units of parts per million (ppm) downfield from $\mathrm{SiMe}_{4}(\delta 0.0)$ and relative to the signal of chloroform- $d$ ( $\delta 7.260$, singlet). Multiplicities were given as: s (singlet); brs (broad singlet); $\mathrm{d}$ (doublet); t (triplet); q (quartet); dd (doublets of doublet); ddd (doublets of doublets of doublet); td (triplet of doublet); $\mathrm{m}$ (multiplets); ddt (doublet of doublet of triplet) and etc. Coupling constants are reported as a $J$ value in $\mathrm{Hz}$. Carbon nuclear magnetic resonance spectra $\left({ }^{13} \mathrm{C}\right.$ NMR) are reported as $\delta$ in units of parts per million (ppm) downfield from $\mathrm{SiMe}_{4}(\delta 0.0)$ and relative to the signal of chloroform- $d(\delta 77.00$, triplet). Melting points were tested on Optimelt MPA100 - automated melting point system. All the heating reactions were performed in oil bath on Heidolph hot plate.

Substrates $\mathbf{1 a}{ }^{1}, \mathbf{1 b}^{2}, \mathbf{1 c}^{3}, \mathbf{1} \mathbf{d}^{4}, \mathbf{1} \mathbf{e}^{5}$ and $\mathbf{1 f}-\mathbf{1} \mathbf{v}^{1,6}$ were synthesized from the corresponding phenols according to literature procedures. Diaryliodonium salts were synthesized according to the reported procedure. ${ }^{7}$

\section{Procedure and characterization data}

$(8 R, 9 S, 13 S, 14 S)$-13-methyl-17-oxo-7,8,9,11,12,13,14,15,16,17-decahydro-6Hcyclopenta[a]phenanthren-3-yl dimethylcarbamate (1v)

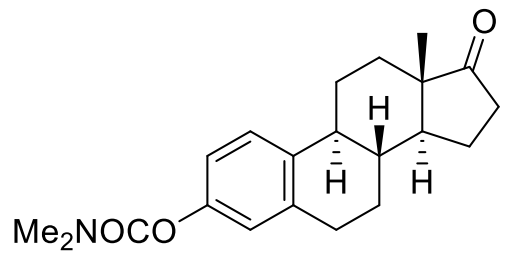

${ }^{1} \mathrm{H} \mathrm{NMR}\left(400 \mathrm{MHz}, \mathrm{CDCl}_{3}\right) \delta 7.30-7.23(\mathrm{~m}, 1 \mathrm{H}), 6.93-6.83(\mathrm{~m}, 2 \mathrm{H}), 3.10(\mathrm{~s}, 3 \mathrm{H}), 3.02$ (s, $3 \mathrm{H}), 2.95-2.88(\mathrm{~m}, 2 \mathrm{H}), 2.52(\mathrm{dd}, J=18.8,8.7 \mathrm{~Hz}, 1 \mathrm{H}), 2.46-2.38(\mathrm{~m}, 1 \mathrm{H}), 2.31-2.26$ (m, 1H), $2.21-1.93(\mathrm{~m}, 4 \mathrm{H}), 1.70-1.39(\mathrm{~m}, 6 \mathrm{H}), 0.92(\mathrm{~s}, 3 \mathrm{H})$.

${ }^{13} \mathrm{C}$ NMR $\left(101 \mathrm{MHz}, \mathrm{CDCl}_{3}\right) \delta 220.7,155.1,149.3,137.6,136.6,126.1,121.7,118.9,50.4$, $47.9,44.0,38.0,36.6,36.3,35.8,31.5,29.3,26.3,25.7,21.5,13.8$.

MP. $=165-167^{\circ} \mathrm{C}$

HRMS: calcd for $\mathrm{C}_{21} \mathrm{H}_{28} \mathrm{NO}_{3}[\mathrm{M}+\mathrm{H}]^{+}:$342.2069, found: 342.2058 . 


\section{1. General Procedure A: Synthesis of $\boldsymbol{m}$-arylated phenols}

To a solution of the appropriate phenol carbamate $(0.30 \mathrm{mmol})$ in 1,2-dichloroethane $(2.0 \mathrm{~mL})$ was added the appropriate iodonium salt $(0.36-0.90 \mathrm{mmol})$ and $\mathrm{Cu}(\mathrm{OTf})_{2}(11 \mathrm{mg}, 0.030$ mmol). The reaction was stirred for the $24 \mathrm{~h}$ at $70{ }^{\circ} \mathrm{C}$ before dilution with $\mathrm{CH}_{2} \mathrm{Cl}_{2}(25 \mathrm{~mL})$ and washing with saturated sodium bicarbonate solution $(15 \mathrm{~mL})$. The aqueous phase was extracted with further $\mathrm{CH}_{2} \mathrm{Cl}_{2}(25 \mathrm{~mL})$ and the combined organics were dried over magnesium sulphate and evaporated in vacuo. The crude residue was dissolved in $\mathrm{EtOH}(5 \mathrm{~mL})$ and 10 equivalents of $\mathrm{NaOH}$ were added. The reaction mixture was heated at $80^{\circ} \mathrm{C}$ for $12 \mathrm{~h}$. After cooling, solvent was evaporated and the crude was acidified with $2 \mathrm{~N} \mathrm{HCl}$ solution. Aqueous layer was further extracted with diethyl ether $(3 \times 25 \mathrm{~mL})$. The combined organic layers were dried, evaporated and purified by flash column chromatography to yield the pure m-arylphenol.

\section{2. General Procedure B: Synthesis of $\boldsymbol{m}$-arylated phenol carbamates}

To a solution of the appropriate phenol carbamate $(0.30 \mathrm{mmol})$ in 1, 2-dichloroethane $(2.0 \mathrm{~mL})$ was added the appropriate iodonium salt $(0.36-0.90 \mathrm{mmol})$ and $\mathrm{Cu}(\mathrm{OTf})_{2}(11 \mathrm{mg}, 0.030$ mmol). The reaction was stirred for the $24 \mathrm{~h}$ at $70{ }^{\circ} \mathrm{C}$ before dilution with $\mathrm{CH}_{2} \mathrm{Cl}_{2}(25 \mathrm{~mL})$ and washing with saturated sodium bicarbonate solution $(15 \mathrm{~mL})$. The aqueous phase was extracted with further $\mathrm{CH}_{2} \mathrm{Cl}_{2}(25 \mathrm{~mL})$ and the combined organics were dried over magnesium sulphate and evaporated in vacuo. The crude residue was dissolved in $\mathrm{MeCN}(10 \mathrm{~mL})$ and 1.5 equivalents of $\mathrm{K}_{2} \mathrm{CO}_{3}$ and 1.2 equivalents of $\mathrm{N}, \mathrm{N}^{\prime}$ - dimethylcarbamoyl chloride were added. The reaction mixture was heated at $85{ }^{\circ} \mathrm{C}$ for $2 \mathrm{~h}$. The reaction mixture was cooled to room temperature and concentrated under a vacuum. The residue was dissolved in $\mathrm{H}_{2} \mathrm{O}$ and extracted with dichloromethane. The organic fractions were combined and then washed successively with $1 \mathrm{M} \mathrm{KOH}$ and water. Finally the organic layer was separated, dried over anhydrous $\mathrm{Na}_{2} \mathrm{SO}_{4}$, concentrated under a vacuum and purified by flash column chromatography to yield the pure m-arylcarbamate.

\section{3. Characterization data}

\section{4-methyl-[1, 1'-biphenyl]-3-ol (2a)}

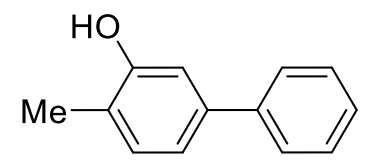

Following the general procedure A, phenol carbamate $1 \mathrm{a}(54.0 \mathrm{mg}, 0.3 \mathrm{mmol})$ was reacted with diphenyliodonium tetrafluoroborate $(134.0 \mathrm{mg} 0.36 \mathrm{mmol})$. The crude product was purified by flash column chromatography (Hexane: Ether 95:5) to afford 4-methyl-[1, 1'biphenyl]-3-ol (2a) as white solid (41.0 mg, 74\%). MP. 76-78 ${ }^{\circ} \mathrm{C}$.

${ }^{1} \mathrm{H}$ NMR (400 MHz, $\left.\mathrm{CDCl}_{3}\right) \delta 7.59-7.53(\mathrm{~m}, 2 \mathrm{H}), 7.44-7.40(\mathrm{~m}, 2 \mathrm{H}), 7.36-7.30(\mathrm{~m}, 1 \mathrm{H})$, $7.19(\mathrm{~d}, J=7.8 \mathrm{~Hz}, 1 \mathrm{H}), 7.11-7.09(\mathrm{~m}, 1 \mathrm{H}), 7.02(\mathrm{~d}, J=1.6 \mathrm{~Hz}, 1 \mathrm{H}), 4.87(\mathrm{~s}, 1 \mathrm{H}), 2.30(\mathrm{~s}$, $3 \mathrm{H})$.

${ }^{13} \mathrm{C} \mathrm{NMR}\left(101 \mathrm{MHz}, \mathrm{CDCl}_{3}\right) \delta 154.1,140.8,140.6,131.4,128.7,127.2,127.0,122.9,119.6$, 113.7, 15.5.

HRMS: calcd for $\mathrm{C}_{13} \mathrm{H}_{13} \mathrm{O}[\mathrm{M}+\mathrm{H}]^{+}:$185.0966, found: 185.0966 . 
Spectroscopic data was in agreement with the previously reported literature. ${ }^{8}$

4-(tert-butyl)-[1, 1'-biphenyl]-3-ol (2f)

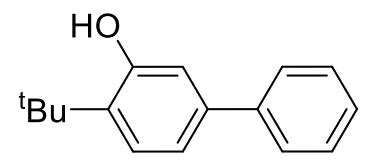

Following the general procedure A, phenol carbamate $\mathbf{1 f}(67.0 \mathrm{mg}, 0.3 \mathrm{mmol})$ was reacted with diphenyliodonium tetrafluoroborate $(134.0 \mathrm{mg} 0.36 \mathrm{mmol})$. The crude product was purified by flash column chromatography (Hexane: Ether 95:5) to afford 4-(tert-butyl)-[1,1'-biphenyl]-3ol (2f) as off white solid (56.8 $\mathrm{mg}, 83 \%)$. MP. $83-85^{\circ} \mathrm{C}$;

${ }^{1} \mathrm{H}$ NMR (400 MHz, $\left.\mathrm{CDCl}_{3}\right) \delta 7.60-7.55(\mathrm{~m}, 2 \mathrm{H}), 7.47-7.41(\mathrm{~m}, 2 \mathrm{H}), 7.39-7.32(\mathrm{~m}, 2 \mathrm{H})$, $7.14(\mathrm{dd}, J=8.1,1.9 \mathrm{~Hz}, 1 \mathrm{H}), 6.90(\mathrm{~d}, J=1.9 \mathrm{~Hz}, 1 \mathrm{H}), 4.91$ (s, 1H), 1.48 (s, 9H).

${ }^{13} \mathrm{C} \mathrm{NMR}\left(101 \mathrm{MHz}, \mathrm{CDCl}_{3}\right) \delta 154.4,140.4,140.3,135.3,128.7,127.6,127.3,126.9,119.3$, 115.2, 34.4, 29.7.

HRMS: calcd for $\mathrm{C}_{16} \mathrm{H}_{19} \mathrm{O}[\mathrm{M}+\mathrm{H}]^{+}:$227.1436, found: 227.1438 .

\section{4-isopropyl-[1, 1'-biphenyl]-3-ol (2g)}

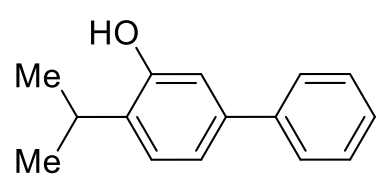

Following the general procedure A, phenol carbamate $1 \mathrm{~g}(62.8 \mathrm{mg}, 0.3 \mathrm{mmol})$ was reacted with diphenyliodonium tetrafluoroborate $(134.0 \mathrm{mg} 0.36 \mathrm{mmol})$. The crude product was purified by flash column chromatography (Hexane: Ether 95:5) to afford 4-isopropyl-[1, 1'biphenyl]-3-ol (2g) as pale yellow solid (50.0 mg, 78\%), MP. 66-68 ${ }^{\circ} \mathrm{C}$.

${ }^{1} \mathrm{H}$ NMR $\left(400 \mathrm{MHz}, \mathrm{CDCl}_{3}\right) \delta 7.54(\mathrm{~d}, J=7.5 \mathrm{~Hz}, 2 \mathrm{H}), 7.40(\mathrm{t}, J=7.4 \mathrm{~Hz}, 2 \mathrm{H}), 7.31(\mathrm{t}, J=$ $7.3 \mathrm{~Hz}, 1 \mathrm{H}), 7.24(\mathrm{~s}, 1 \mathrm{H}), 7.19-7.08(\mathrm{~m}, 2 \mathrm{H}), 6.96(\mathrm{~s}, 1 \mathrm{H}), 4.82(\mathrm{~s}, 1 \mathrm{H}), 3.32-3.17(\mathrm{~m}, 1 \mathrm{H})$, $1.29(\mathrm{~s}, 3 \mathrm{H}), 1.27(\mathrm{~s}, 3 \mathrm{H})$.

${ }^{13} \mathrm{C}$ NMR $\left(101 \mathrm{MHz}, \mathrm{CDCl}_{3}\right) \delta 153.0,140.7,140.0,133.5,128.7,127.2,127.0,126.9,119.8$, 114.0, 27.0, 22.6.

HRMS: calcd for $\mathrm{C}_{15} \mathrm{H}_{17} \mathrm{O}[\mathrm{M}+\mathrm{H}]^{+}:$213.1279, found: 213.1279 .

\section{4-methoxy-[1, 1'-biphenyl]-3-ol (2h)}

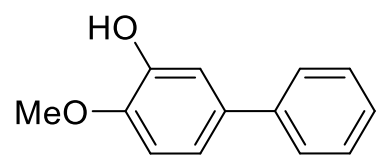


Following the general procedure A, phenol carbamate $\mathbf{1 h}(59.20 \mathrm{mg}, 0.3 \mathrm{mmol})$ was reacted with diphenyliodonium tetrafluoroborate $(134.0 \mathrm{mg} 0.36 \mathrm{mmol})$. The crude product was purified by flash column chromatography (Hexane: Ether 95:5) to afford 4-methoxy-[1, 1'biphenyl]-3-ol (2h) as off white solid (53.4 mg, 88\%). MP. 106-108 ${ }^{\circ} \mathrm{C}$.

${ }^{1} \mathrm{H}$ NMR $\left(400 \mathrm{MHz}, \mathrm{CDCl}_{3}\right) \delta 7.58-7.54(\mathrm{~m}, 2 \mathrm{H}), 7.41(\mathrm{t}, J=7.6 \mathrm{~Hz}, 2 \mathrm{H}), 7.31(\mathrm{t}, J=7.3$ $\mathrm{Hz}, 1 \mathrm{H}), 7.21(\mathrm{~d}, J=2.2 \mathrm{~Hz}, 1 \mathrm{H}), 7.12-7.08(\mathrm{~m}, 1 \mathrm{H}), 6.93(\mathrm{~d}, J=8.3 \mathrm{~Hz}, 1 \mathrm{H}), 5.68(\mathrm{~s}, 1 \mathrm{H})$, $3.94(\mathrm{~s}, 3 \mathrm{H})$.

${ }^{13} \mathrm{C}$ NMR $\left(101 \mathrm{MHz}, \mathrm{CDCl}_{3}\right) \delta 146.2,145.9,140.8,134.9,128.7,126.9,126.8,118.8,113.4$, 111.0, 56.1.

HRMS: calcd for $\mathrm{C}_{13} \mathrm{H}_{13} \mathrm{O}_{2}[\mathrm{M}+\mathrm{H}]^{+}:$201.0916, found: 201.0919 .

Spectroscopic data was in agreement with the previously reported literature ${ }^{9}$

4-chloro-[1, 1'-biphenyl]-3-ol (2i)

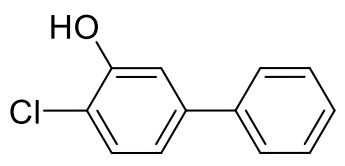

Following the general procedure, phenol carbamate $1 \mathbf{i}(60.5 \mathrm{mg}, 0.3 \mathrm{mmol})$ was reacted with diphenyliodonium tetrafluoroborate $(134.0 \mathrm{mg} 0.36 \mathrm{mmol})$. The crude product was purified by flash column chromatography (Hexane: Ether 95:5) to afford 4-chloro-[1,1'-biphenyl]-3-ol (2i) as as a colorless oil which solidified on standing to give a colorless solid. (40.32 $\mathrm{mg}, 65 \%)$. $\mathrm{MP} .=52-54{ }^{\circ} \mathrm{C}$.

${ }^{1} \mathrm{H}$ NMR (400 MHz, $\left.\mathrm{CDCl}_{3}\right) \delta 7.57-7.54(\mathrm{~m}, 2 \mathrm{H}), 7.47-7.41(\mathrm{~m}, 2 \mathrm{H}), 7.40-7.34(\mathrm{~m}, 2 \mathrm{H})$, $7.27(\mathrm{~d}, J=2.1 \mathrm{~Hz}, 1 \mathrm{H}), 7.11(\mathrm{dd}, J=8.3,2.1 \mathrm{~Hz}, 1 \mathrm{H}), 5.63(\mathrm{~s}, 1 \mathrm{H})$.

${ }^{13} \mathrm{C} \mathrm{NMR}\left(101 \mathrm{MHz}, \mathrm{CDCl}_{3}\right) \delta 151.5,142.0,139.9,129.2,128.9,127.8,127.0,120.2,119.0$, 114.9 .

HRMS: calcd for $\mathrm{C}_{12} \mathrm{H}_{10} \mathrm{ClO}[\mathrm{M}+\mathrm{H}]^{+}:$205.0420, found: 205.0422 .

Spectroscopic data was in agreement with the previously reported literature. ${ }^{8}$

\section{4-bromo-[1, 1'-biphenyl]-3-ol (2j)}

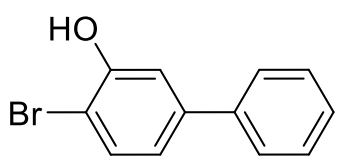

Following the general procedure A, phenol carbamate $\mathbf{1 j}(74.0 \mathrm{mg}, 0.3 \mathrm{mmol})$ was reacted with diphenyliodonium tetrafluoroborate $(134.0 \mathrm{mg} 0.36 \mathrm{mmol})$. The crude product was purified by flash column chromatography (Hexane: Ether 95:5) to afford 4-bromo-[1, 1'-biphenyl]-3-ol $(\mathbf{2 j})$ as brown solid $(45.3 \mathrm{mg}, 60 \%)$. MP. $=182-184{ }^{\circ} \mathrm{C}$. 
${ }^{1} \mathrm{H}$ NMR $\left(400 \mathrm{MHz}, \mathrm{CDCl}_{3}\right) \delta 7.58-7.56(\mathrm{~m}, 2 \mathrm{H}), 7.52(\mathrm{~d}, J=8.3 \mathrm{~Hz}, 1 \mathrm{H}), 7.47-7.41(\mathrm{~m}$, 2H), $7.39-7.36(\mathrm{~m}, 1 \mathrm{H}), 7.27(\mathrm{~d}, J=2.1 \mathrm{~Hz}, 1 \mathrm{H}), 7.06(\mathrm{dd}, J=8.3,2.1 \mathrm{~Hz}, 1 \mathrm{H}), 5.59(\mathrm{~s}, 1 \mathrm{H})$.

${ }^{13} \mathrm{C}$ NMR $\left(101 \mathrm{MHz}, \mathrm{CDCl}_{3}\right) \delta 152.5,142.7,139.8,132.2,128.9,127.8,127.0,120.7,114.7$, 109.3.

HRMS: calcd for $\mathrm{C}_{12} \mathrm{H}_{10} \mathrm{BrO}[\mathrm{M}+\mathrm{H}]^{+}: 248.9915$, found: 248.9905 .

\section{$\left[1,1^{\prime}: 4,1^{\prime \prime}\right.$-terphenyl]-2'-ol (2k)}

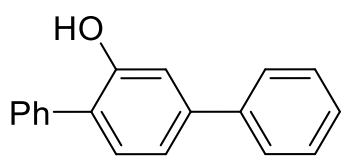

Following the general procedure A, phenol carbamate $1 \mathbf{k}(73.0 \mathrm{mg}, 0.3 \mathrm{mmol})$ was reacted with diphenyliodonium tetrafluoroborate $(134.0 \mathrm{mg} 0.36 \mathrm{mmol})$. The crude product was purified by flash column chromatography (Hexane: Ether 95:5) to afford [1, 1':4, 1'terphenyl]-2'-ol (2k) as off white solid (57.4 mg, 77\%) MP. $174-176{ }^{\circ} \mathrm{C}$;

${ }^{1} \mathrm{H}$ NMR (400 MHz, $\left.\mathrm{CDCl}_{3}\right) \delta 7.58(\mathrm{~d}, J=7.1 \mathrm{~Hz}, 2 \mathrm{H}), 7.46(\mathrm{~s}, 4 \mathrm{H}), 7.43-7.34(\mathrm{~m}, 3 \mathrm{H}), 7.32$ $-7.26(\mathrm{~m}, 2 \mathrm{H}), 7.19(\mathrm{~s}, 2 \mathrm{H}), 5.25(\mathrm{~s}, 1 \mathrm{H})$.

${ }^{13} \mathrm{C} \mathrm{NMR}\left(101 \mathrm{MHz}, \mathrm{CDCl}_{3}\right) \delta 152.7,142.4,140.5,136.9,130.6,129.4,129.1,128.8,128.0$, 127.6, 127.1, 127.1, 119.7, 114.5.

HRMS: calcd for $\mathrm{C}_{18} \mathrm{H}_{15} \mathrm{O}[\mathrm{M}+\mathrm{H}]^{+}:$247.1123, found: 247.1120 .

4-cyclohexyl-[1, 1'-biphenyl]-3-ol (2l)

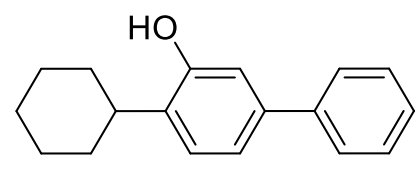

Following the general procedure A, phenol carbamate 11 (75.0 $\mathrm{mg}, 0.3 \mathrm{mmol})$ was reacted with diphenyliodonium tetrafluoroborate $(134.0 \mathrm{mg} 0.36 \mathrm{mmol})$. The crude product was purified by flash column chromatography (Hexane: Ether 95:5) to afford 4-cyclohexyl-[1, 1'-biphenyl]-3ol (2l) as off white solid (55.0 mg, $72 \%)$ MP. $85-87^{\circ} \mathrm{C}$.

${ }^{1} \mathrm{H}$ NMR (400 MHz, $\left.\mathrm{CDCl}_{3}\right) \delta 7.57-7.54(\mathrm{~m}, 2 \mathrm{H}), 7.45-7.39(\mathrm{~m}, 2 \mathrm{H}), 7.35-7.29(\mathrm{~m}, 1 \mathrm{H})$, $7.21-7.10(\mathrm{~m}, 2 \mathrm{H}), 6.99$ (d, $J=1.8 \mathrm{~Hz}, 1 \mathrm{H}), 4.81(\mathrm{~s}, 1 \mathrm{H}), 2.90-2.80(\mathrm{~m}, 1 \mathrm{H}), 1.96-1.85$ $(\mathrm{m}, 4 \mathrm{H}), 1.83-1.75(\mathrm{~m}, 1 \mathrm{H}), 1.51-1.40(\mathrm{~m}, 4 \mathrm{H}), 1.36-1.25(\mathrm{~m}, 1 \mathrm{H})$.

${ }^{13} \mathrm{C} \mathrm{NMR}\left(101 \mathrm{MHz}, \mathrm{CDCl}_{3}\right) \delta 153.0,140.7,139.9,132.7,128.7,127.4,127.2,127.0,119.8$, 114.0, 37.2, 33.2, 27.1, 26.3.

HRMS: calcd for $\mathrm{C}_{18} \mathrm{H}_{21} \mathrm{O}[\mathrm{M}+\mathrm{H}]^{+}:$253.1592, found: 253.1589 . 


\section{4-methoxy-[1, 1':4, 1'"-terphenyl]-2'-ol (2m)}

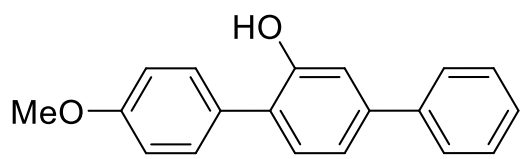

Following the general procedure A, phenol carbamate $1 \mathbf{m}(81.3 \mathrm{mg}, 0.3 \mathrm{mmol})$ was reacted with diphenyliodonium tetrafluoroborate $(134.0 \mathrm{mg} 0.36 \mathrm{mmol})$. The crude product was purified by flash column chromatography (Hexane: Ether 95:5) to afford 4-methoxy-[1, 1':4, $1^{\prime \prime}$-terphenyl]-2'-ol (2m) as brown solid (46.3 mg, 56\%) MP. 100-102 ${ }^{\circ} \mathrm{C}$;

${ }^{1} \mathrm{H}$ NMR $\left(400 \mathrm{MHz}, \mathrm{CDCl}_{3}\right) \delta 7.63(\mathrm{~d}, J=7.3 \mathrm{~Hz}, 2 \mathrm{H}), 7.47-7.42(\mathrm{~m}, 4 \mathrm{H}), 7.36(\mathrm{t}, J=7.3$ $\mathrm{Hz}, 1 \mathrm{H}), 7.31-7.28(\mathrm{~m}, 1 \mathrm{H}), 7.25-7.21(\mathrm{~m}, 2 \mathrm{H}), 7.05(\mathrm{~d}, J=8.7 \mathrm{~Hz}, 1 \mathrm{H}), 5.31(\mathrm{~s}, 1 \mathrm{H}), 3.87$ (s, 3H).

${ }^{13} \mathrm{C} \mathrm{NMR}\left(101 \mathrm{MHz}, \mathrm{CDCl}_{3}\right) \delta 159.5,152.8,142.0,140.6,130.6,130.3,129.0,128.8,127.5$, $127.2,127.1,126.9,119.6,114.8,114.3,55.4$.

HRMS: calcd for $\mathrm{C}_{19} \mathrm{H}_{17} \mathrm{O}_{2}[\mathrm{M}+\mathrm{H}]^{+}:$277.1229, found: 277.1231 .

5-(tert-butyl)-[1, 1'-biphenyl]-3-ol (2n)

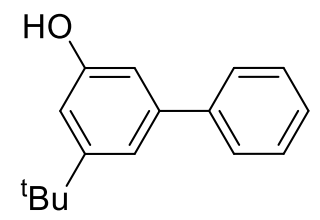

Following the general procedure A, phenol carbamate 1n $(67.0 \mathrm{mg}, 0.3 \mathrm{mmol})$ was reacted with diphenyliodonium tetrafluoroborate $5 \mathrm{a}(156.0 \mathrm{mg} 0.36 \mathrm{mmol})$. The crude product was purified by flash column chromatography (Hexane: Ether 95:5) to afford 5-(tert-butyl)-[1, 1'biphenyl]-3-ol (2n) as off white solid (50.7 mg, 74\%) MP. 82-84 ${ }^{\circ} \mathrm{C}$;

${ }^{1} \mathrm{H}$ NMR $\left(400 \mathrm{MHz}, \mathrm{CDCl}_{3}\right) \delta 7.59-7.55(\mathrm{~m}, 2 \mathrm{H}), 7.46-7.41(\mathrm{~m}, 2 \mathrm{H}), 7.38-7.32(\mathrm{~m}, 1 \mathrm{H})$, $7.19(\mathrm{t}, J=1.6 \mathrm{~Hz}, 1 \mathrm{H}), 6.88-6.87(\mathrm{~m}, 2 \mathrm{H}), 4.82(\mathrm{~s}, 1 \mathrm{H}), 1.36(\mathrm{~s}, 9 \mathrm{H})$.

${ }^{13} \mathrm{C}$ NMR $\left(101 \mathrm{MHz}, \mathrm{CDCl}_{3}\right) \delta 155.6,153.7,142.6,141.4,128.7,127.4,127.3,117.2,111.6$, 111.3, 34.9, 31.4.

HRMS: calcd for $\mathrm{C}_{16} \mathrm{H}_{19} \mathrm{O}[\mathrm{M}+\mathrm{H}]^{+}:$227.1436, found: 227.1444 .

\section{5-methyl-[1, 1'-biphenyl]-3-ol (2o)}

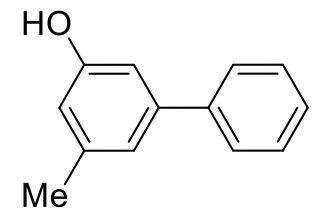


Following the general procedure A, phenol carbamate $10(54.0 \mathrm{mg}, 0.3 \mathrm{mmol})$ was reacted with diphenyliodonium tetrafluoroborate $5 \mathrm{a}(156.0 \mathrm{mg} 0.36 \mathrm{mmol})$. The crude product was purified by flash column chromatography (Hexane: Ether 95:5) to afford 5-methyl-[1, 1'biphenyl]-3-ol (2o) as pale yellow oil (36.7 $\mathrm{mg}, 66 \%)$.

${ }^{1} \mathrm{H}$ NMR $\left(400 \mathrm{MHz}, \mathrm{CDCl}_{3}\right) \delta 7.58-7.54(\mathrm{~m}, 2 \mathrm{H}), 7.44-7.40(\mathrm{~m}, 2 \mathrm{H}), 7.36-7.30(\mathrm{~m}, 1 \mathrm{H})$, $6.99(\mathrm{~s}, 1 \mathrm{H}), 6.87(\mathrm{~s}, 1 \mathrm{H}), 6.65(\mathrm{~s}, 1 \mathrm{H}), 4.83(\mathrm{~s}, 1 \mathrm{H}), 2.37$ (s, 3H).

${ }^{13} \mathrm{C} \mathrm{NMR}\left(101 \mathrm{MHz}, \mathrm{CDCl}_{3}\right) \delta 154.0,140.8,140.6,131.4,128.7,127.2,127.0,122.9,119.6$, $113.7,15.5$.

HRMS: calcd for $\mathrm{C}_{13} \mathrm{H}_{13} \mathrm{O}[\mathrm{M}+\mathrm{H}]^{+}:$185.0966, found: 185.0969 .

\section{5-methoxy-[1, 1'-biphenyl]-3-ol (2p) and 4-methoxy-[1,1'-biphenyl]-2-ol (2po}
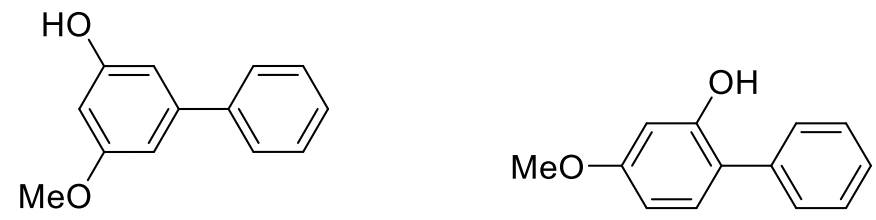

Following the general procedure A, phenol carbamate 1 p (59.2 mg, $0.3 \mathrm{mmol}$ ) was reacted with diphenyliodonium tetrafluoroborate $5 \mathrm{a}(156.0 \mathrm{mg} 0.36 \mathrm{mmol})$. The crude product was purified by flash column chromatography (Hexane: Ether 95:5) to afford 5-methoxy-[1, 1'biphenyl]-3-ol (2p) as pale brown oil (40.7 mg, 67\%) and 4-methoxy-[1,1'-biphenyl]-2-ol (2 $\left.\mathbf{p}_{\boldsymbol{o}}\right)$ $(11.5 \mathrm{mg}, 19 \%)$ as brown oil.

\section{Spectral data for $2 p$}

${ }^{1} \mathrm{H}$ NMR $\left(400 \mathrm{MHz}, \mathrm{CDCl}_{3}\right) \delta 7.58-7.53(\mathrm{~m}, 2 \mathrm{H}), 7.42(\mathrm{t}, J=7.4 \mathrm{~Hz}, 2 \mathrm{H}), 7.39-7.31(\mathrm{~m}$, $1 \mathrm{H}), 6.76-6.71(\mathrm{~m}, 1 \mathrm{H}), 6.69-6.64(\mathrm{~m}, 1 \mathrm{H}), 6.43(\mathrm{t}, J=2.2 \mathrm{~Hz}, 1 \mathrm{H}), 5.32(\mathrm{~s}, 1 \mathrm{H}), 3.84(\mathrm{~s}$, $3 \mathrm{H})$.

${ }^{13} \mathrm{C} \mathrm{NMR}\left(101 \mathrm{MHz}, \mathrm{CDCl}_{3}\right) \delta 161.2,156.9,143.8,140.8,128.7,127.7,127.1,107.0,105.7$, $100.4,55.5$.

HRMS: calcd for $\mathrm{C}_{13} \mathrm{H}_{13} \mathrm{O}_{2}[\mathrm{M}+\mathrm{H}]^{+}$: 201.0916, found: 201.0918 .

\section{Spectral data for $2 \mathbf{p}_{o}$}

${ }^{1} \mathrm{H}$ NMR $\left(400 \mathrm{MHz}, \mathrm{CDCl}_{3}\right) \delta 7.51-7.42(\mathrm{~m}, 4 \mathrm{H}), 7.40-7.34(\mathrm{~m}, 1 \mathrm{H}), 7.16(\mathrm{dd}, J=7.2,1.7$ $\mathrm{Hz}, 1 \mathrm{H}), 6.58$ (dd, $J=7.6,2.0 \mathrm{~Hz}, 2 \mathrm{H}), 5.29$ (s, 1H), 3.83 (s, 3H).

${ }^{13} \mathrm{C} \mathrm{NMR}\left(101 \mathrm{MHz}, \mathrm{CDCl}_{3}\right) \delta 160.6,153.4,137.0,130.8,129.4,129.1,127.6,120.9,107.0$, 101.3, 55.4.

HRMS: calcd for $\mathrm{C}_{13} \mathrm{H}_{13} \mathrm{O}_{2}[\mathrm{M}+\mathrm{H}]^{+}$: 201.0916, found: 201.0909 . 


\section{5-fluoro-[1, 1'-biphenyl]-3-ol (2q)}

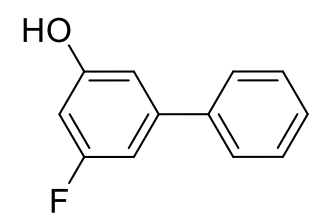

Following the general procedure A, phenol carbamate $1 \mathrm{q}(55.5 \mathrm{mg}, 0.3 \mathrm{mmol})$ was reacted with diphenyliodonium tetrafluoroborate $5 \mathrm{a}(156.0 \mathrm{mg} 0.36 \mathrm{mmol})$. The crude product was purified by flash column chromatography (Hexane: Ether 95:5) to afford 5-fluoro-[1, 1'biphenyl]-3-ol (2q) as brown oil (31.9 $\mathrm{mg}, 56 \%)$.

${ }^{1} \mathrm{H}$ NMR $\left(400 \mathrm{MHz}, \mathrm{CDCl}_{3}\right) \delta 7.56-7.53(\mathrm{~m}, 2 \mathrm{H}), 7.47-7.42(\mathrm{~m}, 2 \mathrm{H}), 7.41-7.35(\mathrm{~m}, 1 \mathrm{H})$, $6.93-6.88(\mathrm{~m}, 1 \mathrm{H}), 6.88-6.84(\mathrm{~m}, 1 \mathrm{H}), 6.58(\mathrm{dt}, J=9.8,2.3 \mathrm{~Hz}, 1 \mathrm{H}), 5.50(\mathrm{~s}, 1 \mathrm{H})$.

${ }^{13} \mathrm{C}$ NMR $\left(101 \mathrm{MHz}, \mathrm{CDCl}_{3}\right) \delta 163.91(\mathrm{~d}, J=245.0 \mathrm{~Hz}), 157.0(\mathrm{~d}, J=12.2 \mathrm{~Hz}), 144.3(\mathrm{~d}, J=$ $9.8 \mathrm{~Hz}), 139.7(\mathrm{~d}, J=2.6 \mathrm{~Hz}), 128.9,128.1,127.0,110.0(\mathrm{~d}, J=2.6 \mathrm{~Hz}), 106.7$ (d, $J=22.4$ $\mathrm{Hz}), 102.0(\mathrm{~d}, J=24.8 \mathrm{~Hz})$.

${ }^{19} \mathrm{~F}$ NMR $\left(377 \mathrm{MHz}, \mathrm{CDCl}_{3}\right) \delta-111.60$.

HRMS: calcd for $\mathrm{C}_{12} \mathrm{H}_{10} \mathrm{FO}[\mathrm{M}+\mathrm{H}]^{+}:$189.0716, found: 189.0716 .

\section{[1, 1'-biphenyl]-3-ol (2r)}

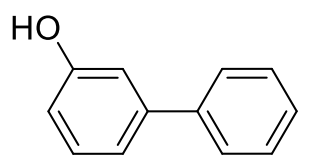

Following the general procedure A, phenol carbamate $1 \mathbf{r}(120.0 \mathrm{mg}, 0.72 \mathrm{mmol})$ was reacted with diphenyliodonium tetrafluoroborate $(134.0 \mathrm{mg} 0.36 \mathrm{mmol})$. The crude product was purified by flash column chromatography (Hexane: Ether 95:5) to afford [1, 1'-biphenyl]-3-ol (2r) as light brown solid $(39.7 \mathrm{mg}, 64 \%) \mathrm{MP} .64-66{ }^{\circ} \mathrm{C}$;

${ }^{1} \mathrm{H}$ NMR (400 MHz, $\left.\mathrm{CDCl}_{3}\right) \delta 7.52(\mathrm{~d}, J=7.9 \mathrm{~Hz}, 2 \mathrm{H}), 7.38(\mathrm{t}, J=7.5 \mathrm{~Hz}, 2 \mathrm{H}), 7.33-7.24$ (m, 2H), $7.12(\mathrm{~d}, J=7.7 \mathrm{~Hz}, 1 \mathrm{H}), 7.02(\mathrm{~s}, 1 \mathrm{H}), 6.79-6.76(\mathrm{~m}, 1 \mathrm{H}), 5.03(\mathrm{~s}, 1 \mathrm{H})$.

${ }^{13} \mathrm{C} \mathrm{NMR}\left(101 \mathrm{MHz}, \mathrm{CDCl}_{3}\right) \delta 155.9,143.1,140.8,130.0,128.8,127.5,127.1,119.8,114.2$, 114.1.

HRMS: calcd for $\mathrm{C}_{12} \mathrm{H}_{11} \mathrm{O}[\mathrm{M}+\mathrm{H}]^{+}:$171.0810, found: 171.0808 .

Spectroscopic data was in agreement with the previously reported literature. ${ }^{10}$ 


\section{$\left[1,1^{\prime}: 3,1^{\prime \prime}\right.$-terphenyl]-5'-ol (2s)}<smiles>Oc1cc(-c2ccccc2)cc(-c2ccccc2)c1</smiles>

Following the general procedure A, phenol carbamate 1r $(50.0 \mathrm{mg}, 0.30 \mathrm{mmol})$ was reacted with diphenyliodonium tetrafluoroborate $(334.0 \mathrm{mg} 0.90 \mathrm{mmol})$. The crude product was purified by flash column chromatography (Hexane: Ether 95:5) to afford [1, 1':3, 1"terphenyl]-5'-ol (2s) as light brown solid (45.5 mg, 61\%). MP. $69-71{ }^{\circ} \mathrm{C}$;

${ }^{1} \mathrm{H}$ NMR $\left(400 \mathrm{MHz}, \mathrm{CDCl}_{3}\right) \delta 7.63(\mathrm{~d}, J=7.3 \mathrm{~Hz}, 4 \mathrm{H}), 7.46(\mathrm{t}, J=7.5 \mathrm{~Hz}, 4 \mathrm{H}), 7.40-7.36$ $(\mathrm{m}, 3 \mathrm{H}), 7.06(\mathrm{~d}, J=1.3 \mathrm{~Hz}, 1 \mathrm{H}), 5.21(\mathrm{~s}, 1 \mathrm{H})$.

${ }^{13} \mathrm{C}$ NMR $\left(101 \mathrm{MHz}, \mathrm{CDCl}_{3}\right) \delta 156.2,143.5,140.8,128.8,127.7,127.2,119.0,113.1$.

HRMS: calcd for $\mathrm{C}_{18} \mathrm{H}_{15} \mathrm{O}[\mathrm{M}+\mathrm{H}]^{+}:$247.1123, found: 247.1126 .

Spectroscopic data was in agreement with the previously reported literature ${ }^{11}$

$2^{\prime}$-ethyl-[1, 1':3, 1'-terphenyl]-5'-ol (2t)<smiles>CCc1c(-c2ccccc2)cc(O)cc1-c1ccccc1</smiles>

Following the general procedure A, phenol carbamate $1 \mathrm{t}(58.5 \mathrm{mg}, 0.3 \mathrm{mmol})$ was reacted with diphenyliodonium tetrafluoroborate $5 \mathrm{a}(334.0 \mathrm{mg} 0.90 \mathrm{mmol})$. The crude product was purified by flash column chromatography (Hexane: Ether 95:5) to afford 2'-ethyl-[1, 1':3, 1'terphenyl]-5'-ol (2t) as light orange gummy solid (49.8 $\mathrm{mg}, 60 \%)$.

${ }^{1} \mathrm{H} \mathrm{NMR}\left(400 \mathrm{MHz}, \mathrm{CDCl}_{3}\right) \delta 7.42-7.33(\mathrm{~m}, 10 \mathrm{H}), 6.69(\mathrm{~s}, 2 \mathrm{H}), 4.70(\mathrm{~s}, 1 \mathrm{H}), 2.47$ (q, J = 7.4 $\mathrm{Hz}, 2 \mathrm{H}), 0.65(\mathrm{t}, J=7.4 \mathrm{~Hz}, 3 \mathrm{H})$.

${ }^{13} \mathrm{C} \mathrm{NMR}\left(101 \mathrm{MHz}, \mathrm{CDCl}_{3}\right) \delta 152.4,143.9,142.3,132.1,129.2,128.0,126.9,116.4,22.3$, 15.5 .

HRMS: calcd for $\mathrm{C}_{20} \mathrm{H}_{19} \mathrm{O}[\mathrm{M}+\mathrm{H}]^{+}:$275.1436, found: 275.1437 . 
<smiles>Cc1cc(Cl)c(O)cc1-c1ccccc1</smiles>

Following the general procedure A, phenol carbamate $1 \mathbf{u}(94.0 \mathrm{mg}, 0.3 \mathrm{mmol})$ was reacted with diphenyliodonium tetrafluoroborate $5 \mathrm{a}(134.0 \mathrm{mg} 0.36 \mathrm{mmol})$. The crude product was purified by flash column chromatography (Hexane: Ether 95:5) to afford 4-((3r,5r,7r)adamantan-1-yl)-6-methyl-[1,1'-biphenyl]-3-ol (2u) as white solid (61.1 mg, 64\%). MP. 155$157{ }^{\circ} \mathrm{C}$.

${ }^{1} \mathrm{H}$ NMR $\left(400 \mathrm{MHz}, \mathrm{CDCl}_{3}\right) \delta 7.41-7.38(\mathrm{~m}, 2 \mathrm{H}), 7.34-7.31(\mathrm{~m}, 3 \mathrm{H}), 7.10(\mathrm{~s}, 1 \mathrm{H}), 6.55(\mathrm{~s}$, $1 \mathrm{H}), 4.66(\mathrm{~s}, 1 \mathrm{H}), 2.22(\mathrm{~s}, 3 \mathrm{H}), 2.18-2.17(\mathrm{~m}, 6 \mathrm{H}), 2.11(\mathrm{~s}, 3 \mathrm{H}), 1.81(\mathrm{~s}, 6 \mathrm{H})$.

${ }^{13} \mathrm{C} \mathrm{NMR}\left(101 \mathrm{MHz}, \mathrm{CDCl}_{3}\right) \delta 152.1,141.4,140.0,135.3,129.2,129.1,128.1,127.0,126.7$, 118.2, 40.7, 37.1, 36.5, 29.1, 19.8 .

HRMS: calcd for $\mathrm{C}_{23} \mathrm{H}_{27} \mathrm{O}[\mathrm{M}+\mathrm{H}]^{+}: 319.2062$, found: 319.2066 .

$(8 R, 9 S, 13 S, 14 S)$-3-hydroxy-13-methyl-1-phenyl-6,7,8,9,11,12,13,14,15,16-decahydro$17 H$-cyclopenta[a]phenanthren-17-one $(2 \mathrm{v})$<smiles>C[C@]12CC[C@H]3c4c(cc(O)cc4-c4ccccc4)CC[C@H]3[C@@H]1CCC2=O</smiles>

Following the general procedure A estrone carbamate $1 \mathbf{v}(102.3 \mathrm{mg}, 0.3 \mathrm{mmol})$ was reacted with diphenyliodonium tetrafluoroborate $5 \mathrm{a}(134.0 \mathrm{mg} 0.36 \mathrm{mmol})$. The crude product was purified by flash column chromatography (Hexane: Ether $75: 25)$ to afford $(8 R, 9 S, 13 S, 14 S)$-3hydroxy-13-methyl-1-phenyl-6,7,8,9,11,12,13,14,15,16-decahydro-17H-

cyclopenta[a]phenanthren-17-one $(2 \mathbf{v})$ as off white solid $(63.3 \mathrm{mg}, 61 \%) . \mathrm{MP} .=159-161{ }^{\circ} \mathrm{C}$.

${ }^{1} \mathrm{H}$ NMR $\left(400 \mathrm{MHz}, \mathrm{CDCl}_{3}\right) \delta 7.37-7.26(\mathrm{~m}, 5 \mathrm{H}), 6.60(\mathrm{~d}, J=2.5 \mathrm{~Hz}, 1 \mathrm{H}), 6.56(\mathrm{~d}, J=2.6$ $\mathrm{Hz}, 1 \mathrm{H}), 5.29(\mathrm{~s}, 1 \mathrm{H}), 3.00-2.81(\mathrm{~m}, 2 \mathrm{H}), 2.66(\mathrm{t}, J=8.5 \mathrm{~Hz}, 1 \mathrm{H}), 2.45(\mathrm{dd}, J=18.6,8.2 \mathrm{~Hz}$, $1 \mathrm{H}), 2.13-1.98(\mathrm{~m}, 2 \mathrm{H}), 1.89(\mathrm{dd}, J=12.2,4.9 \mathrm{~Hz}, 1 \mathrm{H}), 1.62-1.51(\mathrm{~m}, 3 \mathrm{H}), 1.46-1.35(\mathrm{~m}$, $3 \mathrm{H}), 1.07-0.88(\mathrm{~m}, 3 \mathrm{H}), 0.82(\mathrm{~s}, 3 \mathrm{H})$.

${ }^{13} \mathrm{C} \mathrm{NMR}\left(101 \mathrm{MHz}, \mathrm{CDCl}_{3}\right) \delta 221.3,153.1,144.0,143.9,140.2,129.3,128.5,127.7,126.9$, $116.7,114.8,50.5,48.4,45.4,40.6,35.8,32.0,29.7,26.8,25.1,21.6,14.3$.

HRMS: calcd for $\mathrm{C}_{24} \mathrm{H}_{27} \mathrm{O}_{2}[\mathrm{M}+\mathrm{H}]^{+}$: 347.2011, found: 347.2005. 


\section{4, 4'-dimethyl-[1, 1'-biphenyl]-3-ol (2w)}

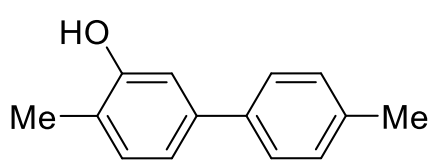

Following the general procedure A, phenol carbamate $1 \mathrm{a}(54.0 \mathrm{mg}, 0.3 \mathrm{mmol})$ was reacted with $p$-tolyl(mesityl)iodonium trifluoromethanesulfonate $(176.0 \mathrm{mg} 0.36 \mathrm{mmol})$. The crude product was purified by flash column chromatography (Hexane: Ether 95:5) to afford 4, 4'dimethyl-[1,1'-biphenyl]-3-ol (2w) as pale yellow solid (47.8 mg, 80\%) MP. 84-86 ${ }^{\circ} \mathrm{C}$;

${ }^{1} \mathrm{H}$ NMR $\left(400 \mathrm{MHz}, \mathrm{CDCl}_{3}\right) \delta 7.46(\mathrm{~d}, J=8.1 \mathrm{~Hz}, 2 \mathrm{H}), 7.23(\mathrm{~d}, J=8.0 \mathrm{~Hz}, 2 \mathrm{H}), 7.17(\mathrm{~d}, J=$ $7.8 \mathrm{~Hz}, 1 \mathrm{H}), 7.08(\mathrm{dd}, J=7.7,1.7 \mathrm{~Hz}, 0 \mathrm{H}), 7.00(\mathrm{~d}, J=1.6 \mathrm{~Hz}, 1 \mathrm{H}), 4.77$ (s, 1H), 2.39 (s, 3H), $2.29(\mathrm{~s}, 3 \mathrm{H})$.

${ }^{13} \mathrm{C}$ NMR (101 MHz, $\left.\mathrm{CDCl}_{3}\right) \delta 154.0,140.5,137.9,137.0,131.3,129.5,126.8,122.5,119.4$, 113.5, 21.1, 15.5.

HRMS: calcd for $\mathrm{C}_{14} \mathrm{H}_{15} \mathrm{O}[\mathrm{M}+\mathrm{H}]^{+}:$199.1123, found: 199.1120 .

\section{4'-fluoro-4-methyl-[1,1'-biphenyl]-3-ol (2x)}

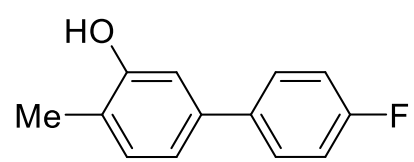

Following the general procedure A, phenol carbamate $1 \mathrm{a}(54.0 \mathrm{mg}, 0.3 \mathrm{mmol})$ was reacted with 4-fluorophenyl(mesityl)iodonium trifluoromethanesulfonate (177.0 $\mathrm{mg} 0.36 \mathrm{mmol})$. The crude product was purified by flash column chromatography (Hexane: Ether 95:5) to afford 4'fluoro-4-methyl-[1,1'-biphenyl]-3-ol (2x) as brown solid (41.5 mg, 68\%) MP. 60-62 ${ }^{\circ} \mathrm{C}$;

${ }^{1} \mathrm{H}$ NMR $\left(400 \mathrm{MHz}, \mathrm{CDCl}_{3}\right) \delta 7.53-7.47(\mathrm{~m}, \mathrm{H}), 7.18(\mathrm{~d}, J=7.7 \mathrm{~Hz}, 1 \mathrm{H}), 7.13-7.07(\mathrm{~m}$, 2H), $7.04(\mathrm{dd}, J=7.7,1.7 \mathrm{~Hz}, 1 \mathrm{H}), 6.96(\mathrm{~d}, J=1.7 \mathrm{~Hz}, 1 \mathrm{H}), 4.90(\mathrm{~s}, 1 \mathrm{H}), 2.29(\mathrm{~s}, 3 \mathrm{H})$.

${ }^{13} \mathrm{C}$ NMR $\left(101 \mathrm{MHz}, \mathrm{CDCl}_{3}\right) \delta 162.4(\mathrm{~d}, J=246.1 \mathrm{~Hz}), 154.1,139.6,136.9(\mathrm{~d}, J=3.3 \mathrm{~Hz})$, $131.4,128.5(\mathrm{~d}, J=8.0 \mathrm{~Hz}), 122.8,119.4,115.6(\mathrm{~d}, J=21.4 \mathrm{~Hz}), 113.6,15.4$.

${ }^{19} \mathrm{~F}$ NMR $\left(377 \mathrm{MHz}, \mathrm{CDCl}_{3}\right) \delta-115.88$.

HRMS: calcd for $\mathrm{C}_{13} \mathrm{H}_{12} \mathrm{FO}[\mathrm{M}+\mathrm{H}]^{+}:$203.0872, found: 203.0872 .

\section{4'-chloro-4-methyl-[1,1'-biphenyl]-3-ol (2y)}

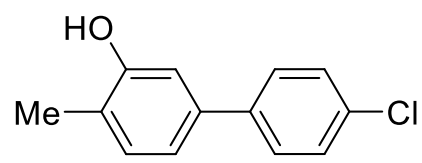

Following the general procedure A, phenol carbamate $1 \mathrm{a}(54.0 \mathrm{mg}, 0.3 \mathrm{mmol})$ was reacted with 4-chloro phenyl(mesityl)iodonium trifluoromethanesulfonate (183.0 mg $0.36 \mathrm{mmol}$ ). The 
crude product was purified by flash column chromatography (Hexane: Ether 95:5) to afford 4' chloro-4-methyl-[1,1'-biphenyl]-3-ol (2y) as brown solid (42.8 mg, 65\%) MP. 66-68 ${ }^{\circ} \mathrm{C}$;

${ }^{1} \mathrm{H} \mathrm{NMR}\left(400 \mathrm{MHz}, \mathrm{CDCl}_{3}\right) \delta 7.48(\mathrm{~d}, J=8.4 \mathrm{~Hz}, 2 \mathrm{H}), 7.38(\mathrm{~d}, J=8.4 \mathrm{~Hz}, 2 \mathrm{H}), 7.18(\mathrm{~d}, J=$ $7.7 \mathrm{~Hz}, 1 \mathrm{H}), 7.05$ (d, $J=7.8 \mathrm{~Hz}, 1 \mathrm{H}), 6.98(\mathrm{~s}, 1 \mathrm{H}), 4.85$ (s, 1H), 2.29 (s, 3H).

${ }^{13} \mathrm{C}$ NMR $\left(101 \mathrm{MHz}, \mathrm{CDCl}_{3}\right) \delta 154.2,139.3,139.2,133.3,131.5,128.9,128.2,123.2,119.4$, $113.5,15.5$.

HRMS: calcd for $\mathrm{C}_{13} \mathrm{H}_{12} \mathrm{ClO}[\mathrm{M}+\mathrm{H}]^{+}:$219.0577, found: 219.0575 .

\section{4'-bromo-4-methyl-[1,1'-biphenyl]-3-ol (2z)}

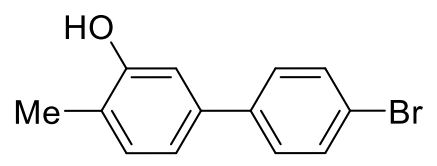

Following the general procedure A, phenol carbamate 1a $(54.0 \mathrm{mg}, 0.3 \mathrm{mmol})$ was reacted with 4-bromophenyl(mesityl)iodonium trifluoromethanesulfonate $(199.0 \mathrm{mg} 0.36 \mathrm{mmol})$. The crude product was purified by flash column chromatography (Hexane: Ether 95:5) to afford 4'bromo-4-methyl-[1,1'-biphenyl]-3-ol (2z) as brown solid (48.3 mg, 61\%) MP. 93-95 ${ }^{\circ} \mathrm{C}$;

${ }^{1} \mathrm{H} \mathrm{NMR}\left(400 \mathrm{MHz}, \mathrm{CDCl}_{3}\right) \delta 7.53(\mathrm{~d}, J=8.4 \mathrm{~Hz}, 2 \mathrm{H}), 7.41(\mathrm{~d}, J=8.4 \mathrm{~Hz}, 2 \mathrm{H}), 7.18(\mathrm{~d}, J=$ $7.7 \mathrm{~Hz}, 1 \mathrm{H}), 7.04$ (d, $J=7.8 \mathrm{~Hz}, 1 \mathrm{H}), 6.97$ (s, 1H), 4.90 (s, 1H), 2.29 (s, 3H).

${ }^{13} \mathrm{C} \mathrm{NMR}\left(101 \mathrm{MHz}, \mathrm{CDCl}_{3}\right) \delta 154.2,139.7,139.3,131.8,131.5,128.5,123.3,121.4,119.3$, $113.4,15.5$.

HRMS: calcd for $\mathrm{C}_{13} \mathrm{H}_{12} \mathrm{BrO}[\mathrm{M}+\mathrm{H}]^{+}$: 263.0072, found: 263.0075 .

\section{4-methyl-4'-(trifluoromethyl)-[1,1'-biphenyl]-3-ol (2aa)}

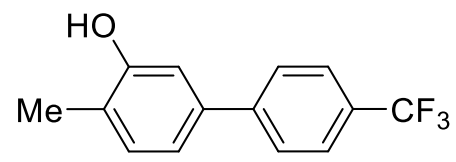

Following the general procedure A, phenol carbamate $1 \mathrm{a}(54.0 \mathrm{mg}, 0.3 \mathrm{mmol})$ was reacted with 4-(trifluoromethyl)phenyl(mesityl)iodonium trifluoromethanesulfonate (195.0 $\mathrm{mg} 0.36$ mmol). The crude product was purified by flash column chromatography (Hexane: Ether 95:5) to afford 4-methyl-4'-(trifluoromethyl)-[1,1'-biphenyl]-3-ol (2aa) as off white solid (43.3 mg, $57 \%)$ MP. $75-76^{\circ} \mathrm{C}$;

${ }^{1} \mathrm{H}$ NMR $\left(400 \mathrm{MHz}, \mathrm{CDCl}_{3}\right) \delta 7.68-7.62(\mathrm{~m}, 4 \mathrm{H}), 7.22(\mathrm{~d}, J=7.7 \mathrm{~Hz}, 1 \mathrm{H}), 7.10(\mathrm{dd}, J=7.7$, $1.7 \mathrm{~Hz}, 1 \mathrm{H}), 7.03(\mathrm{~d}, J=1.6 \mathrm{~Hz}, 1 \mathrm{H}), 4.93(\mathrm{~s}, 1 \mathrm{H}), 2.31$ (s, 3H).

${ }^{19} \mathrm{~F} \mathrm{NMR}\left(377 \mathrm{MHz}, \mathrm{CDCl}_{3}\right) \delta-62.37$.

${ }^{13} \mathrm{C} \mathrm{NMR}\left(101 \mathrm{MHz}, \mathrm{CDCl}_{3}\right) \delta 154.3,144.3,139.0,131.6,127.2,125.7$ (q, $\left.J=3.8 \mathrm{~Hz}\right), 124.0$, 119.7, 113.7, 15.5. 
HRMS: calcd for $\mathrm{C}_{14} \mathrm{H}_{12} \mathrm{~F}_{3} \mathrm{O}[\mathrm{M}+\mathrm{H}]^{+}:$253.0840, found: 253.0842 .

3', 4-dimethyl-[1,1'-biphenyl]-3-ol (2ab)<smiles>Cc1cccc(-c2ccc(C)c(O)c2)c1</smiles>

Following the general procedure A, phenol carbamate 1a $(54.0 \mathrm{mg}, 0.3 \mathrm{mmol})$ was reacted with $m$-tolyl(mesityl)iodonium trifluoromethanesulfonate $(176.0 \mathrm{mg} 0.36 \mathrm{mmol})$. The crude product was purified by flash column chromatography (Hexane: Ether 95:5) to afford 3', 4dimethyl-[1,1'-biphenyl]-3-ol (2ab) as pale yellow oil (42.4 mg, 71\%).

${ }^{1} \mathrm{H}$ NMR $\left(400 \mathrm{MHz}, \mathrm{CDCl}_{3}\right) \delta 7.37(\mathrm{~d}, J=10.6 \mathrm{~Hz}, 2 \mathrm{H}), 7.32(\mathrm{t}, J=7.5 \mathrm{~Hz}, 1 \mathrm{H}), 7.18(\mathrm{dd}, J$ $=11.4,7.6 \mathrm{~Hz}, 2 \mathrm{H}), 7.10(\mathrm{dd}, J=7.7,1.5 \mathrm{~Hz}, 1 \mathrm{H}), 7.02(\mathrm{~d}, J=1.3 \mathrm{~Hz}, 1 \mathrm{H}), 4.94(\mathrm{~s}, 1 \mathrm{H}), 2.42$ $(\mathrm{s}, 3 \mathrm{H}), 2.31(\mathrm{~s}, 3 \mathrm{H})$.

${ }^{13} \mathrm{C} \mathrm{NMR}\left(101 \mathrm{MHz}, \mathrm{CDCl}_{3}\right) \delta 154.0,140.7,140.6,138.3,131.3,128.6,128.0,127.8,124.1$, $122.8,119.5,113.7,21.6,15.5$.

HRMS: calcd for $\mathrm{C}_{14} \mathrm{H}_{15} \mathrm{O}[\mathrm{M}+\mathrm{H}]^{+}:$199.1123, found: 199.1123 .

\section{3'-bromo-4-methyl-[1,1'-biphenyl]-3-ol (2ac)}<smiles>Cc1ccc(-c2cccc(Br)c2)cc1O</smiles>

Following the general procedure A, phenol carbamate $1 \mathrm{a}(54.0 \mathrm{mg}, 0.3 \mathrm{mmol})$ was reacted with 3-bromophenyl (mesityl)iodonium trifluoromethanesulfonate (199.0 $\mathrm{mg} 0.36 \mathrm{mmol}$ ). The crude product was purified by flash column chromatography (Hexane: Ether 95:5) to afford 3'bromo-4-methyl-[1,1'-biphenyl]-3-ol (2ac) as yellow oil (42.8 mg, 54\%).

${ }^{1} \mathrm{H}$ NMR $\left(400 \mathrm{MHz}, \mathrm{CDCl}_{3}\right) \delta 7.70(\mathrm{~s}, 1 \mathrm{H}), 7.46(\mathrm{t}, J=8.2 \mathrm{~Hz}, 2 \mathrm{H}), 7.29(\mathrm{~d}, J=7.8 \mathrm{~Hz}, 1 \mathrm{H})$, $7.26(\mathrm{~s}, 1 \mathrm{H}), 7.19$ (d, $J=7.7 \mathrm{~Hz}, 1 \mathrm{H}), 7.05$ (d, $J=7.8 \mathrm{~Hz}, 1 \mathrm{H}), 6.98$ (s, 1H), 4.89 (s, 1H), 2.29 (s, 3H).

${ }^{13} \mathrm{C}$ NMR $\left(101 \mathrm{MHz}, \mathrm{CDCl}_{3}\right) \delta 154.2,142.9,139.0,131.5,130.3,130.1,130.0,125.6,123.6$, $122.9,119.5,113.6,15.5$.

HRMS: calcd for $\mathrm{C}_{13} \mathrm{H}_{12} \mathrm{BrO}[\mathrm{M}+\mathrm{H}]^{+}:$263.0072, found: 263.0073 .

3'-methoxy-4-methyl-[1,1'-biphenyl]-3-ol (2ad)<smiles>COc1cccc(-c2ccc(C)c(O)c2)c1</smiles> 
Following the general procedure A, phenol carbamate $1 \mathrm{a}(54.0 \mathrm{mg}, 0.3 \mathrm{mmol})$ was reacted with 3-methoxyphenyl (mesityl)iodonium trifluoromethanesulfonate (182.0 mg $0.36 \mathrm{mmol})$. The crude product was purified by flash column chromatography (Hexane: Ether 85:15) to afford 3'-methoxy-4-methyl-[1,1'-biphenyl]-3-ol (2ad) as yellow oil (39.3 mg, 61\%).

${ }^{1} \mathrm{H}$ NMR $\left(400 \mathrm{MHz}, \mathrm{CDCl}_{3}\right) \delta 7.34(\mathrm{t}, J=7.9 \mathrm{~Hz}, 1 \mathrm{H}), 7.17(\mathrm{dd}, J=15.6,7.8 \mathrm{~Hz}, 2 \mathrm{H}), 7.12-$ $7.07(\mathrm{~m}, 2 \mathrm{H}), 7.01(\mathrm{~d}, J=1.5 \mathrm{~Hz}, 1 \mathrm{H}), 6.91-6.86(\mathrm{~m}, 1 \mathrm{H}), 5.03(\mathrm{~s}, 1 \mathrm{H}), 3.86(\mathrm{~s}, 3 \mathrm{H}), 2.30(\mathrm{~s}$, $3 \mathrm{H})$.

${ }^{13} \mathrm{C} \mathrm{NMR}\left(101 \mathrm{MHz}, \mathrm{CDCl}_{3}\right) \delta 159.9,154.0,142.3,140.3,131.3,129.7,123.1,119.5,113.7$, $112.7,112.7,55.3,15.5$.

HRMS: calcd for $\mathrm{C}_{14} \mathrm{H}_{15} \mathrm{O}_{2}[\mathrm{M}+\mathrm{H}]^{+}:$215.1072, found: 215.1071 .

2'-methoxy-4-methyl-[1,1'-biphenyl]-3-ol (2ae)

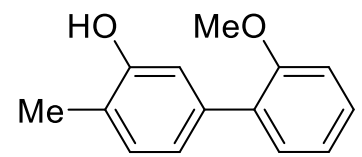

Following the general procedure A, phenol carbamate 1a $(54.0 \mathrm{mg}, 0.3 \mathrm{mmol})$ was reacted with 2-methoxy phenyl(mesityl)iodonium trifluoromethanesulfonate (182.0 mg $0.36 \mathrm{mmol})$. The crude product was purified by flash column chromatography (Hexane: Ether 85:15) to afford 2'-methoxy-4-methyl-[1,1'-biphenyl]-3-ol (2ae) as pale yellow oil (27.0 mg, 42\%).

${ }^{1} \mathrm{H}$ NMR $\left(400 \mathrm{MHz}, \mathrm{CDCl}_{3}\right) \delta 7.35-7.31(\mathrm{~m}, 2 \mathrm{H}), 7.17(\mathrm{~d}, J=7.7 \mathrm{~Hz}, 1 \mathrm{H}), 7.06-6.96(\mathrm{~m}$, $4 \mathrm{H}), 4.93(\mathrm{~s}, 1 \mathrm{H}), 3.82(\mathrm{~s}, 3 \mathrm{H}), 2.30(\mathrm{~s}, 3 \mathrm{H})$.

${ }^{13} \mathrm{C} \mathrm{NMR}\left(101 \mathrm{MHz}, \mathrm{CDCl}_{3}\right) \delta 156.4,153.3,137.6,130.7,130.6,130.3,128.5,122.6,121.9$, $120.8,116.2,111.3,55.6,15.6$.

HRMS: calcd for $\mathrm{C}_{14} \mathrm{H}_{15} \mathrm{O}_{2}[\mathrm{M}+\mathrm{H}]^{+}:$215.1072, found: 215.1066 .

\section{3'-hydroxy-4'-methyl-[1,1'-biphenyl]-4-carboxylic acid (2af)}

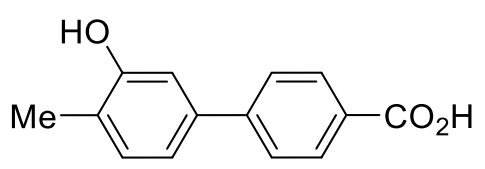

Following the general procedure A, phenol carbamate 1a $(54.0 \mathrm{mg}, 0.3 \mathrm{mmol})$ was reacted with (4-(methoxycarbonyl)phenyl) (mesityl)iodoniumtrifluoromethanesulfonate $(192.0 \mathrm{mg}$ $0.36 \mathrm{mmol}$ ). The crude product was purified by flash column chromatography (Hexane: EA 60:40) to afford 3'-hydroxy-4'-methyl-[1,1'-biphenyl]-4-carboxylic acid (2af) as brown solid (47.4 mg, 69\%) MP. $239-241{ }^{\circ} \mathrm{C}$.

${ }^{1} \mathrm{H}$ NMR (400 MHz, DMSO) $\delta 12.91(\mathrm{~s}, 1 \mathrm{H}), 9.50$ (s, 1H), 7.99 (d, $\left.J=8.3 \mathrm{~Hz}, 2 \mathrm{H}\right), 7.69$ (t, $J$ $=9.3 \mathrm{~Hz}, 2 \mathrm{H}), 7.17(\mathrm{~d}, J=7.8 \mathrm{~Hz}, 1 \mathrm{H}), 7.13-7.01(\mathrm{~m}, 2 \mathrm{H}), 2.16(\mathrm{~s}, 3 \mathrm{H})$.

${ }^{13} \mathrm{C}$ NMR (101 MHz, DMSO) $\delta 167.1,155.8,144.5,137.6,131.2,129.9,129.2,126.3,124.3$, $117.5,112.8,15.7$. 
HRMS: calcd for $\mathrm{C}_{14} \mathrm{H}_{13} \mathrm{O}_{3}[\mathrm{M}+\mathrm{H}]^{+}:$229.0865, found: 229.0864 .

\section{4-methyl-[1,1'-biphenyl]-3-yl dimethylcarbamate (3a)}

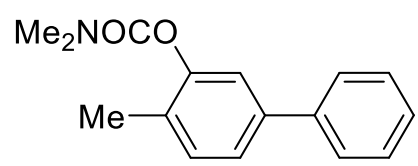

Following the general procedure B, phenol carbamate $1 \mathrm{a}(54.0 \mathrm{mg}, 0.3 \mathrm{mmol})$ was reacted with diphenyliodonium tetrafluoroborate $(134.0 \mathrm{mg} 0.36 \mathrm{mmol})$. The crude product was purified by flash column chromatography (Hexane: Ether 90:10) to afford 4-methyl-[1,1'-biphenyl]-3-yl dimethylcarbamate (3a) as an yellow oil (59.2 $\mathrm{mg}, 77 \%)$.

${ }^{1} \mathrm{H}$ NMR $\left(400 \mathrm{MHz}, \mathrm{CDCl}_{3}\right) \delta 7.59-7.55(\mathrm{~m}, 2 \mathrm{H}), 7.42-7.39(\mathrm{~m}, 2 \mathrm{H}), 7.37-7.30(\mathrm{~m}, 3 \mathrm{H})$, $7.27(\mathrm{~d}, J=7.5 \mathrm{~Hz}, 1 \mathrm{H}), 3.16(\mathrm{~s}, 3 \mathrm{H}), 3.04(\mathrm{~s}, 3 \mathrm{H}), 2.26(\mathrm{~s}, 3 \mathrm{H})$.

${ }^{13} \mathrm{C} \mathrm{NMR}\left(101 \mathrm{MHz}, \mathrm{CDCl}_{3}\right) \delta 154.7,150.3,140.4,140.2,131.3,129.4,128.7,127.2,127.1$, 124.1, 121.0, 36.8, 36.5, 15.9 .

HRMS: calcd for $\mathrm{C}_{16} \mathrm{H}_{18} \mathrm{NO}_{2}[\mathrm{M}+\mathrm{H}]^{+}: 256.1328$, found: 256.1324 .

\section{[1, 1'-biphenyl]-3-yl dimethylcarbamate (3r)}

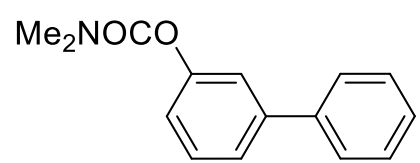

Following the general procedure B, phenol carbamate $1 \mathbf{r}(120.0 \mathrm{mg}, 0.72 \mathrm{mmol})$ was reacted with diphenyliodonium tetrafluoroborate $(134.0 \mathrm{mg} 0.36 \mathrm{mmol})$. The crude product was purified by flash column chromatography (Hexane: Ether 90:10) to afford [1, 1'-biphenyl]-3yl dimethylcarbamate (3r) as light brown oil (60.4 mg, 69\%).

${ }^{1} \mathrm{H}$ NMR $\left(400 \mathrm{MHz}, \mathrm{CDCl}_{3}\right) \delta 7.61-7.59(\mathrm{~m}, 2 \mathrm{H}), 7.47-7.40(\mathrm{~m}, 4 \mathrm{H}), 7.37-7.33(\mathrm{~m}, 2 \mathrm{H})$, $7.15-7.09(\mathrm{~m}, 1 \mathrm{H}), 3.13(\mathrm{~s}, 3 \mathrm{H}), 3.04(\mathrm{~s}, 3 \mathrm{H})$.

${ }^{13} \mathrm{C} \mathrm{NMR}\left(101 \mathrm{MHz}, \mathrm{CDCl}_{3}\right) \delta 154.9,151.9,142.6,140.4,129.5,128.7,127.5,127.2,123.9$, $120.6,120.5,36.7,36.5$.

HRMS: calcd for $\mathrm{C}_{15} \mathrm{H}_{15} \mathrm{NO}_{2}[\mathrm{M}+\mathrm{H}]^{+}:$242.1181, found: 242.1167 .

\section{4-((3r,5r,7r)-adamantan-1-yl)-6-methyl-[1,1'-biphenyl]-3-yl dimethylcarbamate (3u)}

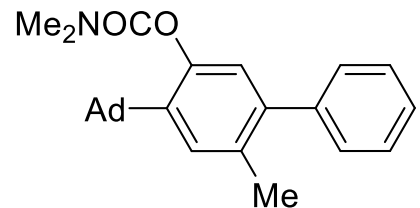

Following the general procedure B, phenol carbamate $\mathbf{3 u}(94.0 \mathrm{mg}, 0.3 \mathrm{mmol})$ was reacted with diphenyliodonium tetrafluoroborate $(134.0 \mathrm{mg} 0.36 \mathrm{mmol})$. The crude product was 
purified by flash column chromatography (Hexane: Ether 90:10) to afford 4-((3r,5r,7r)adamantan-1-yl)-6-methyl-[1,1'-biphenyl]-3-yl dimethylcarbamate (3a) as a white solid (94.6 $\mathrm{mg}, 81 \%)$. MP $=185-187^{\circ} \mathrm{C}$.

${ }^{1} \mathrm{H}$ NMR $\left(400 \mathrm{MHz}, \mathrm{CDCl}_{3}\right) \delta 7.40-7.27(\mathrm{~m}, 5 \mathrm{H}), 7.19(\mathrm{~s}, 1 \mathrm{H}), 6.91(\mathrm{~s}, 1 \mathrm{H}), 3.19(\mathrm{~s}, 3 \mathrm{H})$, 3.05 (s, 3H), 2.28 (s, 3H), $2.10(\mathrm{~d}, J=12.1 \mathrm{~Hz}, 9 \mathrm{H}), 1.79$ (s, 6H).

${ }^{13} \mathrm{C}$ NMR $\left(101 \mathrm{MHz}, \mathrm{CDCl}_{3}\right) \delta 155.2,147.9,141.0,139.9,139.8,131.8,129.3,128.8,127.9$, $126.7,125.7,41.3,37.0,37.0,36.6,36.5,29.0,20.2$.

HRMS: calcd for $\mathrm{C}_{26} \mathrm{H}_{32} \mathrm{NO}_{2}[\mathrm{M}+\mathrm{H}]^{+}: 390.2433$, found: 390.2437 .

\section{3'-methoxy-4-methyl-[1,1'-biphenyl]-3-yl dimethylcarbamate (3ad)}

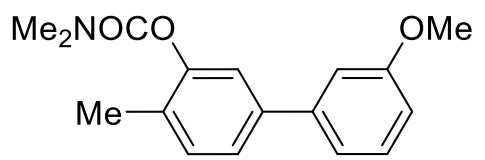

Following the general procedure $\mathrm{B}$, phenol carbamate $1 \mathbf{a}(54.0 \mathrm{mg}, 0.3 \mathrm{mmol})$ was reacted with 3-methoxyphenyl (mesityl)iodonium trifluoromethanesulfonate $(182.0 \mathrm{mg} 0.36 \mathrm{mmol})$. The crude product was purified by flash column chromatography (Hexane: Ether 85:15) to afford 3'-methoxy-4-methyl-[1,1'-biphenyl]-3-yl dimethylcarbamate (3ad) as yellow solid (56.7 mg, $66 \%)$. MP $=82-84{ }^{\circ} \mathrm{C}$.

${ }^{1} \mathrm{H}$ NMR $\left(400 \mathrm{MHz}, \mathrm{CDCl}_{3}\right) \delta 7.37-7.25(\mathrm{~m}, 4 \mathrm{H}), 7.16(\mathrm{~d}, J=7.7 \mathrm{~Hz}, 1 \mathrm{H}), 7.12-7.09(\mathrm{~m}$, $1 \mathrm{H}), 6.89-6.85(\mathrm{~m}, 1 \mathrm{H}), 3.85(\mathrm{~s}, 3 \mathrm{H}), 3.16(\mathrm{~s}, 3 \mathrm{H}), 3.04(\mathrm{~s}, 3 \mathrm{H}), 2.25(\mathrm{~s}, 3 \mathrm{H})$.

${ }^{13} \mathrm{C} \mathrm{NMR}\left(101 \mathrm{MHz}, \mathrm{CDCl}_{3}\right) \delta 159.9,154.6,150.3,141.9,140.1,131.2,129.7,129.6,124.1$, 121.0, 119.6, 112.8, 112.6, 55.3, 36.8, 36.5, 15.9.

HRMS: calcd for $\mathrm{C}_{17} \mathrm{H}_{20} \mathrm{NO}_{3}[\mathrm{M}+\mathrm{H}]^{+}:$286.1443, found: 286.1440 .

\section{2'-methoxy-4-methyl-[1,1'-biphenyl]-3-yl dimethylcarbamate (3ae)}

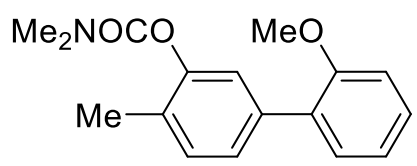

Following the general procedure B, phenol carbamate $1 \mathrm{a}(54.0 \mathrm{mg}, 0.3 \mathrm{mmol})$ was reacted with 2-methoxyphenyl (mesityl)iodonium trifluoromethanesulfonate $(182.0 \mathrm{mg} 0.36 \mathrm{mmol})$. The crude product was purified by flash column chromatography (Hexane: Ether 85:15) to afford 2'-methoxy-4-methyl-[1,1'-biphenyl]-3-yl dimethylcarbamate (3ae) as yellow oil (46.4 mg, $54 \%)$.

${ }^{1} \mathrm{H}$ NMR $\left(400 \mathrm{MHz}, \mathrm{CDCl}_{3}\right) \delta 7.32-7.18(\mathrm{~m}, 5 \mathrm{H}), 6.98-6.91(\mathrm{~m}, 2 \mathrm{H}), 3.76(\mathrm{~s}, 3 \mathrm{H}), 3.11(\mathrm{~s}$, $3 \mathrm{H}), 2.99$ (s, 3H), 2.22 (s, 3H).

${ }^{13} \mathrm{C} \mathrm{NMR}\left(101 \mathrm{MHz}, \mathrm{CDCl}_{3}\right) \delta 156.5,154.7,149.6,137.3,130.9,130.4,129.9,128.9,128.6$, $126.6,123.3,120.8,111.3,55.6,36.7,36.5,16.0$. 
HRMS: calcd for $\mathrm{C}_{17} \mathrm{H}_{20} \mathrm{NO}_{3}[\mathrm{M}+\mathrm{H}]^{+}:$286.1443, found: 286.1434 .

Methyl 3'-((dimethylcarbamoyl)oxy)-4'-methyl-[1,1'-biphenyl]-4-carboxylate (3af)

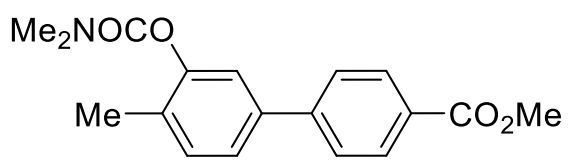

Following the general procedure $\mathrm{B}$, phenol carbamate $1 \mathrm{a}(54.0 \mathrm{mg}, 0.3 \mathrm{mmol})$ was reacted with (4-(methoxycarbonyl)phenyl) (mesityl)iodoniumtrifluoromethanesulfonate (192.0 $\mathrm{mg} 0.36$ mmol). The crude product was purified by flash column chromatography (Hexane: Ether 85:15) to afford 2'-methoxy-4-methyl-[1,1'-biphenyl]-3-yl dimethylcarbamate (3ae) as pale brown solid $(78.3 \mathrm{mg}, 83 \%)$. MP $=67-69^{\circ} \mathrm{C}$.

${ }^{1} \mathrm{H}$ NMR $\left(400 \mathrm{MHz}, \mathrm{CDCl}_{3}\right) \delta 8.07(\mathrm{~d}, J=8.3 \mathrm{~Hz}, 2 \mathrm{H}), 7.63(\mathrm{~d}, J=8.3 \mathrm{~Hz}, 2 \mathrm{H}), 7.40-7.34$ (m, 2H), 7.29 (d, J = 7.8 Hz, 1H), 3.93 (s, 3H), 3.15 (s, 3H), 3.04 (s, 3H), $2.26(\mathrm{~s}, 3 \mathrm{H})$.

${ }^{13} \mathrm{C} \mathrm{NMR}\left(101 \mathrm{MHz}, \mathrm{CDCl}_{3}\right) \delta 167.0,154.6,150.4,144.8,138.8,131.4,130.5,130.08,128.9$, $126.9,124.2,121.1,52.1,36.8,36.5,16.0$.

HRMS: calcd for $\mathrm{C}_{18} \mathrm{H}_{20} \mathrm{NO}_{4}[\mathrm{M}+\mathrm{H}]^{+}: 314.1392$, found: 314.1393 .

\section{Scale up reaction of $m$-arylated phenol carbamate 1a}

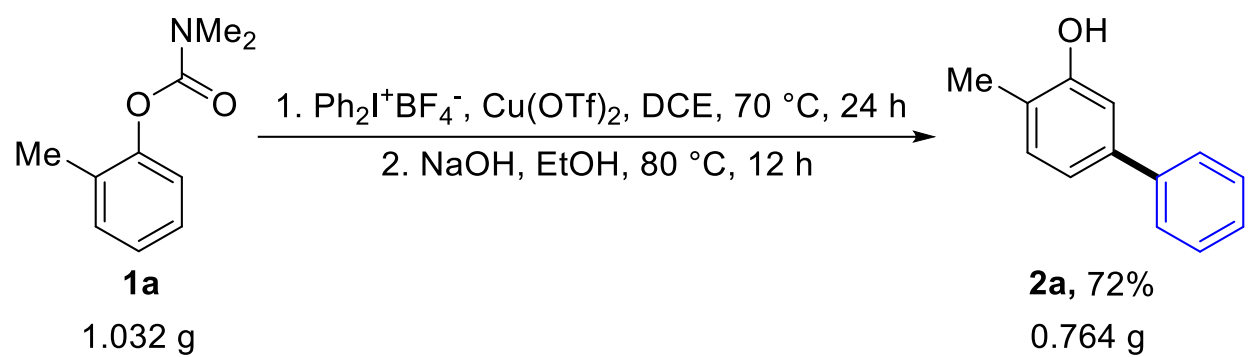

To a solution of the appropriate phenol carbamate $1 \mathrm{a}(1.032 \mathrm{~g}, 5.76 \mathrm{mmol})$ in $1,2-$ dichloroethane $(20.0 \mathrm{~mL})$ was added the diphenyliodonium tetrafluoroborate $(2.54 \mathrm{~g}, 6.90$ mmol, 1.2 equiv) $)$ and $\mathrm{Cu}(\mathrm{OTf})_{2}(0.208 \mathrm{~g}, 0.57 \mathrm{mmol})$. The reaction was stirred for $24 \mathrm{~h}$ at 70 ${ }^{\circ} \mathrm{C}$ before dilution with $\mathrm{CH}_{2} \mathrm{Cl}_{2}(25 \mathrm{~mL})$ and washing with saturated sodium bicarbonate solution $(25 \mathrm{~mL})$. The aqueous phase was extracted with further $\mathrm{CH}_{2} \mathrm{Cl}_{2}(50 \mathrm{~mL})$ and the combined organics were dried over magnesium sulphate and evaporated in vacuo. The crude residue was dissolved in $\mathrm{EtOH}(10 \mathrm{~mL})$ and 10 equivalents of $\mathrm{NaOH}$ were added. The reaction mixture was heated at $80^{\circ} \mathrm{C}$ for $12 \mathrm{~h}$. After cooling, solvent was evaporated and the crude was acidified with $2 \mathrm{~N} \mathrm{HCl}$ solution. Aqueous layer was further extracted with diethyl ether $(3 \mathrm{x} 40$ $\mathrm{mL}$ ). The combined organic layers were dried, evaporated and purified by flash column chromatography to yield the pure m-arylphenol.The crude was purified by flash column chromatography using Hexane: Ether (95:5) to get product 2a in $72 \%$ yield. 


\section{Diversification of 3r}

\section{Reductive cleavage of carbamtes ${ }^{12}$}<smiles>COc1cccc(-c2ccccc2)c1</smiles>

$3 r$

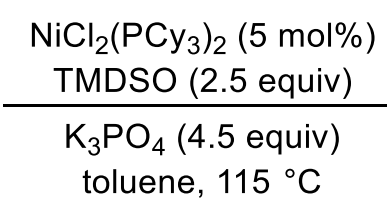<smiles>c1ccc(-c2ccccc2)cc1</smiles>

$4 a, 88 \%$

A 8-mL vial was charged with anhydrous powdered $\mathrm{K}_{3} \mathrm{PO}_{4}(0.289 \mathrm{~g}, 1.36 \mathrm{mmol}, 4.5$ equiv $)$ and a magnetic stir bar. The vial and contents were flame-dried under reduced pressure, then allowed to cool under $\mathrm{N}_{2}$. [1, 1'-biphenyl]-3-yl dimethylcarbamate 3r (73.0, 0.3 mmol, 1 equiv) and $\mathrm{NiCl}_{2}\left(\mathrm{PCy}_{3}\right)_{2} \quad(10.5 \mathrm{mg}, 0.015 \mathrm{mmol}, 5 \mathrm{~mol} \%)$ were added. Subsequently, tetramethyldisiloxane (TMDSO) $(0.14 \mathrm{~mL}, 0.75 \mathrm{mmol}, 2.5$ equiv) and toluene $(1.5 \mathrm{~mL})$ were added. The septum cap was replaced with a Teflon-lined screw cap. The heterogeneous mixture was stirred at $115^{\circ} \mathrm{C}$ for $24 \mathrm{~h}$. The reaction vessel was cooled to $23{ }^{\circ} \mathrm{C}$. The reaction mixture was diluted with EtOAc $(5 \mathrm{~mL})$, filtered over a pad of celite (eluted with an additional $5 \mathrm{~mL}$ of EtOAc), and evaporated to dryness. The crude residue was purified by flash chromatography to yield deoxygenated product $\mathbf{4 a}\left(41.0 \mathrm{mg}, 88 \%\right.$ yield) as a white solid. MP. $70-72{ }^{\circ} \mathrm{C}$;

Spectral data for 4a:

${ }^{1} \mathrm{H}$ NMR (400 MHz, $\left.\mathrm{CDCl}_{3}\right) \delta 7.77-7.74(\mathrm{~m}, 4 \mathrm{H}), 7.62-7.56(\mathrm{~m}, 4 \mathrm{H}), 7.52-7.46(\mathrm{~m}, 2 \mathrm{H})$.

${ }^{13} \mathrm{C} \mathrm{NMR}\left(101 \mathrm{MHz}, \mathrm{CDCl}_{3}\right) \delta 141.2,128.8,127.3,127.2$.

HRMS: calcd for $\mathrm{C}_{12} \mathrm{H}_{11}[\mathrm{M}+\mathrm{H}]^{+}:$155.0861, found: 155.0861 .

\section{Suzuki-Miyaura coupling of carbamtes ${ }^{13}$}<smiles>CC(=O)Oc1cccc(-c2ccccc2)c1</smiles>

$3 r$

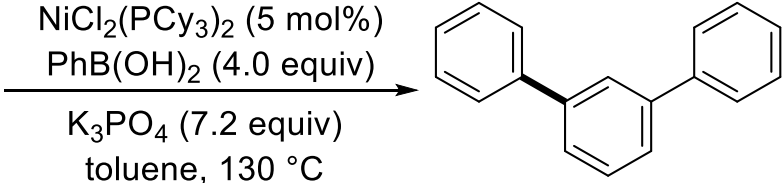

4b, $71 \%$

A 8-mL vial was charged with anhydrous powdered $\mathrm{K}_{3} \mathrm{PO}_{4}(0.462 \mathrm{~g}, 2.18 \mathrm{mmol}, 7.2$ equiv $)$ and a magnetic stir bar. The vial and contents were flame-dried under reduced pressure, then allowed to cool under $\mathrm{N}_{2}$. [1, 1'-biphenyl]-3-yl dimethylcarbamate $3 \mathbf{r}$ (73.0 mg, $0.3 \mathrm{mmol}, 1$ equiv), phenylboronic acid (0.147 g, $1.2 \mathrm{mmol}, 4$ equiv) and $\mathrm{NiCl}_{2}\left(\mathrm{PCy}_{3}\right)_{2}(21.0 \mathrm{mg}, 0.03$ mmol, $10 \mathrm{~mol} \%$ ) were added. The vial was then evacuated and back filled with $\mathrm{N}_{2}$ and toluene $(1.5 \mathrm{~mL})$ was added. The septum cap was replaced with a Teflon-lined screw cap. The heterogeneous mixture was allowed to stir at room temperature for $1 \mathrm{~h}$, then heated to $130{ }^{\circ} \mathrm{C}$ for $24 \mathrm{~h}$. The reaction vessel was cooled to $23^{\circ} \mathrm{C}$. The reaction mixture was diluted with DCM, silica gel was added and evaporated to dryness. The crude residue was purified by flash chromatography to yield product $\mathbf{4 b}$ (41.0 mg, $71 \%$ yield) as a white solid. MP. $88-90{ }^{\circ} \mathrm{C}$; 
Spectral data for $\mathbf{4 b}$ :

${ }^{1} \mathrm{H}$ NMR $\left(400 \mathrm{MHz}, \mathrm{CDCl}_{3}\right) \delta 7.88-7.87(\mathrm{~m}, 1 \mathrm{H}), 7.75-7.68(\mathrm{~m}, 4 \mathrm{H}), 7.66-7.61(\mathrm{~m}, 2 \mathrm{H})$, $7.59-7.48(\mathrm{~m}, 5 \mathrm{H}), 7.42(\mathrm{t}, J=7.4 \mathrm{~Hz}, 2 \mathrm{H})$.

${ }^{13} \mathrm{C} \mathrm{NMR}\left(101 \mathrm{MHz}, \mathrm{CDCl}_{3}\right) \delta 141.8,141.2,129.2,128.8,127.4,127.3,126.2,126.2$.

HRMS: calcd for $\mathrm{C}_{18} \mathrm{H}_{15}[\mathrm{M}+\mathrm{H}]^{+}:$231.1174, found: 231.1177 .

\section{DFT calculations}

Density functional theory (DFT) calculations were performed for a theoretical mechanistic study of the $\mathrm{Cu}$-catalyzed meta-arylation reaction. Gaussian 09 software was used for all DFT calculations. $^{14}$ M06-2X/[Lanl2dz(Cu),6-31G*(other atoms)] (method 1) was used for geometry optimization, whereas M06-2X/def2-TZVP (method 2) was used for single-point energy evaluations. ${ }^{15-18}$ Molecular energies obtained with these methods are referred to as E1 and E2, respectively. Free energy corrections $\left(\mathrm{G}_{\mathrm{corr}}\right)$, as obtained from frequency calculations with method 1 , were added to $\mathrm{E} 2$, and $\mathrm{E} 2+\mathrm{G}_{\text {corr }}(=\mathrm{G})$ values were used to compare relative stability of different species. The closed-shell singlet spin state was assumed for all species.

\section{Raw energy values}

Table S1. Energy values obtained from DFT calculations

\begin{tabular}{|l|r|r|r|r|}
\hline & E1 (hartrees) & $\mathrm{G}_{\text {corr }}$ (hartrees) & E2 (hartrees) & $\Delta \mathrm{G}(\mathrm{kcal} / \mathrm{mol})$ \\
\hline (Mechanism A) & & & & 19.7 \\
\hline Int1Aa & -2850.577090 & 0.253145 & -4294.585955 & 7.0 \\
\hline Int1As & -2850.599719 & 0.257029 & -4294.610063 & 61.2 \\
\hline TS1Aa & -2850.516521 & 0.256691 & -4294.523349 & 55.2 \\
\hline TS1As & -2850.528976 & 0.258259 & -4294.534565 & -16.7 \\
\hline Int2Aa & -2850.647799 & 0.258682 & -4294.649553 & -19.3 \\
\hline Int2As & -2850.654159 & 0.261870 & -4294.656794 & 0.0 \\
\hline Mechanism B) & & & & 11.1 \\
\hline Int1 & -2850.608548 & 0.255077 & -4294.619310 & 17.9 \\
\hline TS1 (meta) & -2850.595089 & 0.256583 & -4294.603095 & -21.9 \\
\hline TS1 (ortho) & -2850.580536 & 0.253426 & -4294.589145 & -20.2 \\
\hline TS1 (para) & -2850.575047 & 0.254182 & -4294.583499 & -61.7 \\
\hline Int2 & -2850.661264 & 0.256128 & -4294.663425 & -4294.651044 \\
\hline TS2 & -2850.647219 & 0.254645 & -429.716117 & \\
\hline Int3 & -2850.708700 & 0.253576 & -4294.7161 & \\
\hline
\end{tabular}

DFT optimized geometries 
(a) Mechanism A

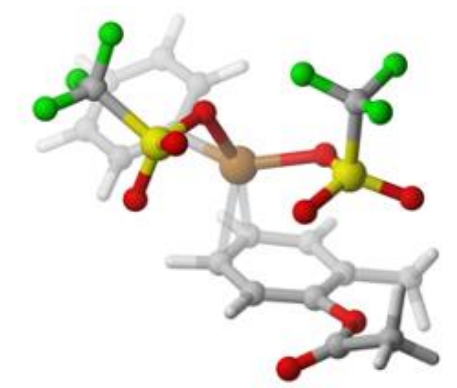

Int1Aa

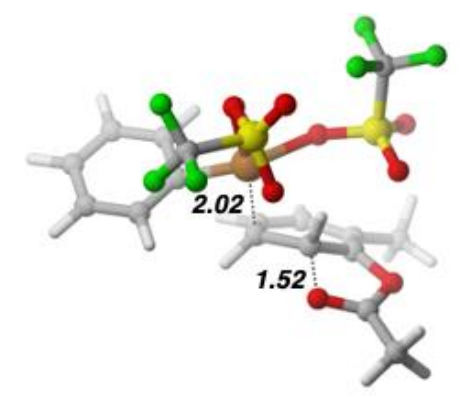

TS1Aa

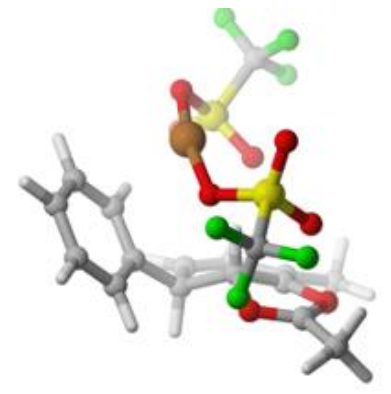

Int2Aa

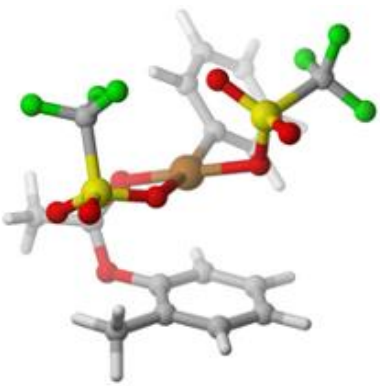

Int1As

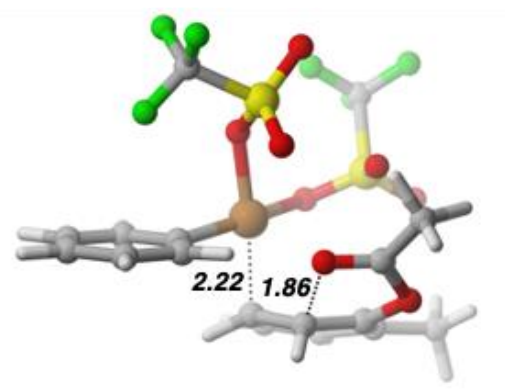

TS1As

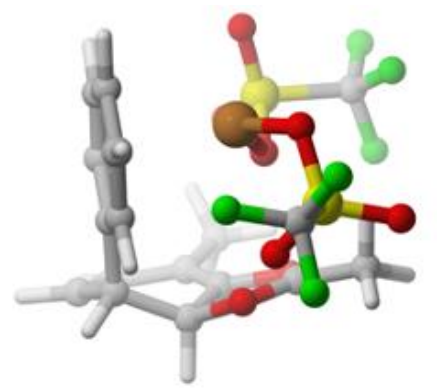

Int2As 
(b) Mechanism B

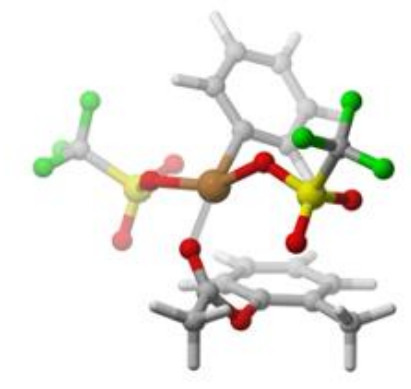

Int1

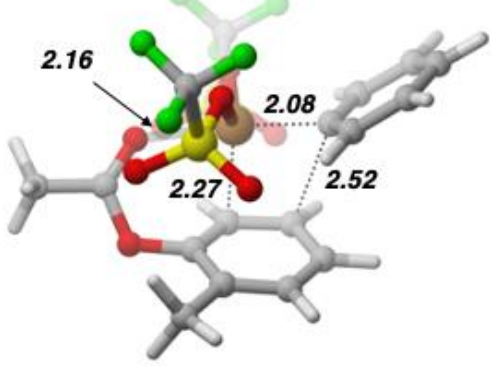

TS1

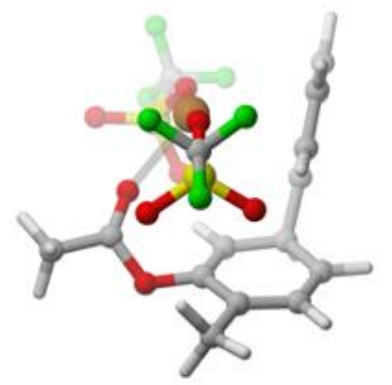

Int2

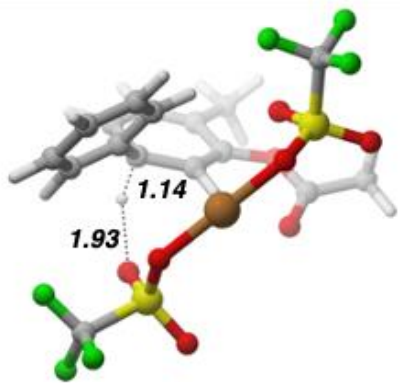

TS2

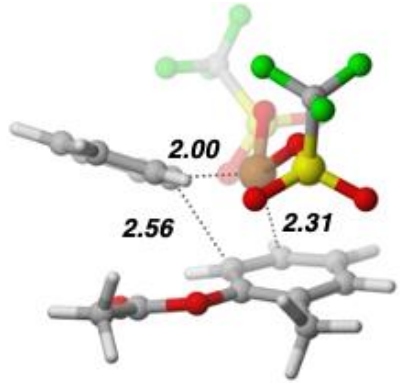

TS1 (ortho)

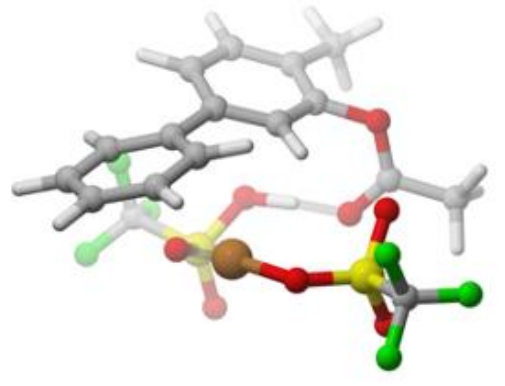

Int3

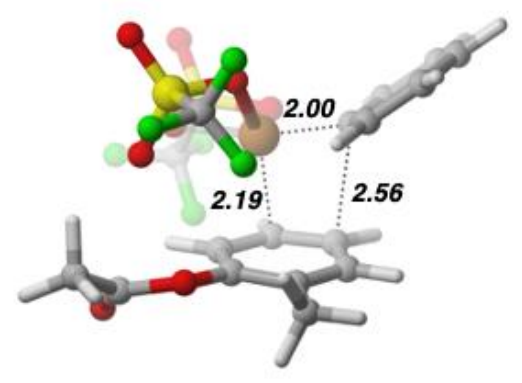

TS1 (para)

Figure S1. M06-2X/B1-optimized geometries for (a) mechanism B (b) mechanism A. Key geometric parameters are shown in $\AA$. 


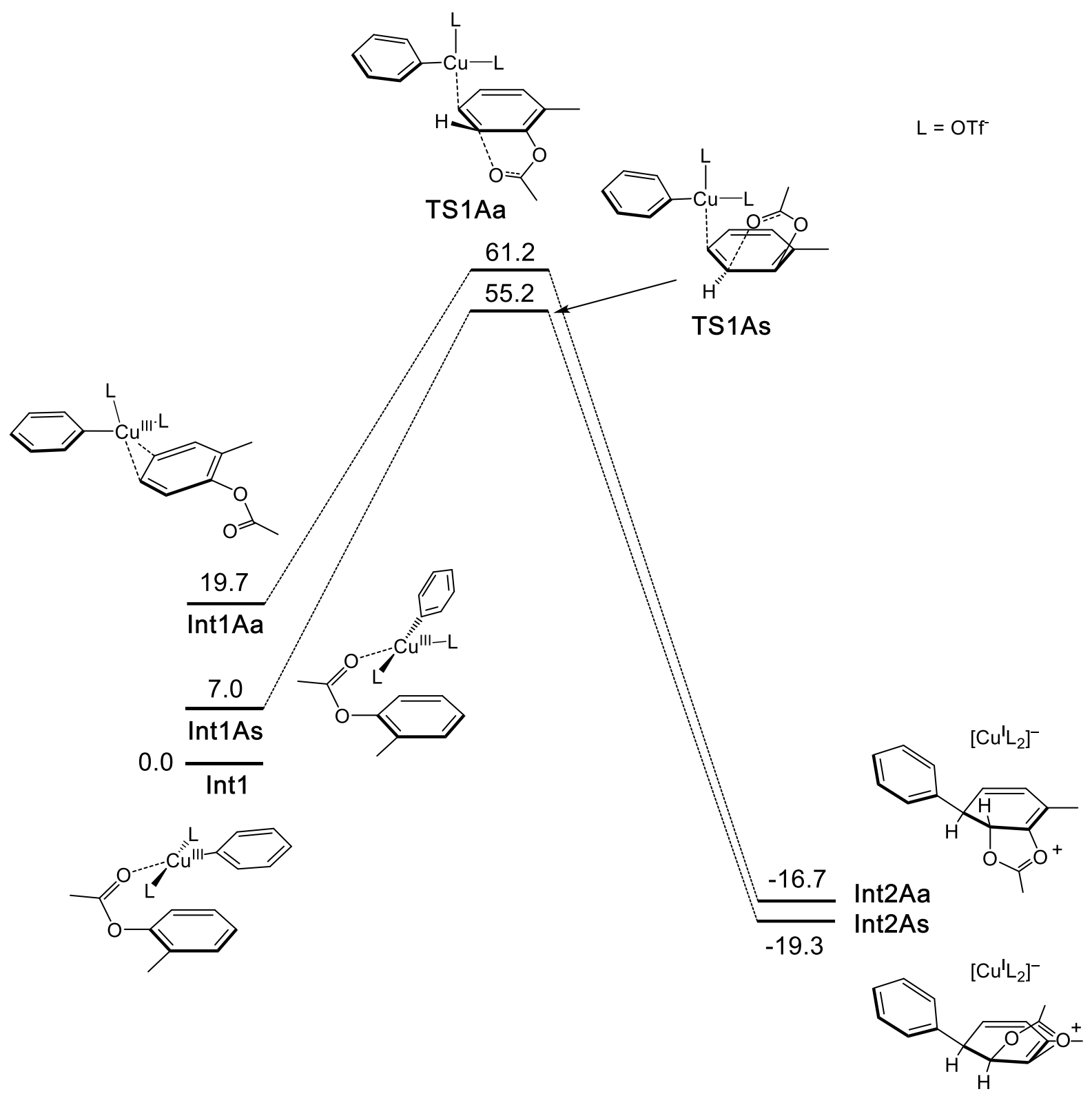

Figure S2. DFT-calculated free energy profile (in $\mathrm{kcal} / \mathrm{mol}$ ) for mechanism A. 


\begin{tabular}{|c|c|c|c|c|c|c|c|}
\hline (Mechanis & sm B) & & & C & -2.715943 & 1.448554 & 2.064145 \\
\hline & & & & $\mathrm{H}$ & -3.625526 & -0.278830 & 2.972111 \\
\hline$===\operatorname{Int} 1$ & $===$ & & & $\mathrm{H}$ & 0.631176 & 1.959936 & 1.786105 \\
\hline $\mathrm{Cu}$ & 0.353339 & 0.009477 & -0.468553 & $\mathrm{H}$ & -1.522988 & 3.055834 & 1.240620 \\
\hline 0 & 0.034307 & -1.854212 & -0.699944 & $\mathrm{H}$ & -3.665837 & 1.927390 & 1.845090 \\
\hline 0 & 0.865879 & 3.800104 & 0.236565 & 0 & 0.887882 & -0.392279 & 3.009468 \\
\hline S & -0.448425 & -2.786771 & 0.403112 & $\mathrm{C}$ & 1.856891 & -0.622734 & 2.127341 \\
\hline 0 & 0.524620 & -2.935688 & 1.477728 & 0 & 1.811752 & -0.296277 & 0.949279 \\
\hline 0 & -1.838604 & -2.562702 & 0.763037 & C & 3.018116 & -1.343123 & 2.728721 \\
\hline C & -0.400868 & -4.374704 & -0.538302 & $\mathrm{H}$ & 2.785692 & -2.412384 & 2.671235 \\
\hline $\mathrm{F}$ & -0.803070 & -5.359132 & 0.259425 & $\mathrm{H}$ & 3.915568 & -1.140823 & 2.144836 \\
\hline $\mathrm{F}$ & -1.209860 & -4.308024 & -1.589191 & $\mathrm{H}$ & 3.152776 & -1.066175 & 3.774836 \\
\hline $\mathrm{F}$ & 0.834939 & -4.627629 & -0.952893 & C & -1.468222 & -1.734780 & 3.772788 \\
\hline S & 0.458832 & 3.100733 & -0.968772 & $\mathrm{H}$ & -1.267548 & -1.538470 & 4.832805 \\
\hline O & 1.031556 & 1.685774 & -1.037738 & $\mathrm{H}$ & -2.434305 & -2.237742 & 3.689853 \\
\hline O & -0.929545 & 3.177709 & -1.388929 & $\mathrm{H}$ & -0.694518 & -2.408125 & 3.401257 \\
\hline C & 1.439320 & 3.842392 & -2.346641 & & & & \\
\hline $\mathrm{F}$ & 1.086820 & 5.113345 & -2.502284 & $===\operatorname{TS} 1$ & $===$ & & \\
\hline $\mathrm{F}$ & 2.739984 & 3.778327 & -2.088689 & $\mathrm{Cu}$ & 0.274375 & 0.170567 & 0.289182 \\
\hline $\mathrm{F}$ & 1.193110 & 3.186153 & -3.483367 & 0 & 0.177215 & -1.840779 & 0.006796 \\
\hline C & -1.139030 & 0.202239 & -1.701065 & 0 & 2.564175 & 2.870055 & 0.829790 \\
\hline C & -2.341215 & 0.025059 & -1.075636 & S & -0.125303 & -2.802432 & 1.115839 \\
\hline C & -0.858391 & 0.539814 & -2.995915 & 0 & 0.850635 & -2.787457 & 2.204483 \\
\hline C & -3.447227 & 0.291135 & -1.897566 & 0 & -1.535394 & -2.791971 & 1.512070 \\
\hline $\mathrm{H}$ & -2.453421 & -0.321453 & -0.051917 & C & 0.124475 & -4.413193 & 0.253121 \\
\hline C & -1.996868 & 0.774992 & -3.777786 & F & -0.150273 & -5.410316 & 1.090836 \\
\hline $\mathrm{H}$ & 0.145857 & 0.678265 & -3.378824 & F & -0.687770 & -4.499143 & -0.798170 \\
\hline C & -3.272888 & 0.661457 & -3.228534 & F & 1.381698 & -4.530842 & -0.159338 \\
\hline $\mathrm{H}$ & -4.441268 & 0.176193 & -1.476176 & S & 1.634147 & 2.852688 & -0.290273 \\
\hline $\mathrm{H}$ & -1.861729 & 1.078335 & -4.811482 & 0 & 1.369373 & 1.490717 & -0.859418 \\
\hline $\mathrm{H}$ & -4.141673 & 0.861003 & -3.847467 & 0 & 0.391792 & 3.620085 & -0.118631 \\
\hline C & -2.693027 & 0.207627 & 2.696985 & C & 2.504468 & 3.665875 & -1.697397 \\
\hline C & -1.491775 & -0.441576 & 3.005995 & F & 2.823248 & 4.914430 & -1.368009 \\
\hline C & -0.317337 & 0.209682 & 2.624048 & F & 3.613819 & 3.004432 & -2.007623 \\
\hline C & -0.307708 & 1.458821 & 2.004656 & F & 1.706483 & 3.696196 & -2.766742 \\
\hline C & -1.522471 & 2.081784 & 1.719681 & C & -1.536834 & 0.543005 & -0.656092 \\
\hline
\end{tabular}




\begin{tabular}{|c|c|c|c|c|c|c|c|}
\hline C & -2.339199 & -0.568748 & -0.620362 & C & -0.428459 & -4.728901 & 0.383676 \\
\hline C & -1.508038 & 1.574548 & -1.556729 & $\mathrm{~F}$ & -0.792730 & -5.354049 & 1.503463 \\
\hline C & -3.159956 & -0.709858 & -1.747553 & $\mathrm{~F}$ & -1.312053 & -5.009101 & -0.570237 \\
\hline $\mathrm{H}$ & -2.331086 & -1.304653 & 0.179472 & $\mathrm{~F}$ & 0.766513 & -5.176109 & 0.012749 \\
\hline C & -2.357198 & 1.383966 & -2.659598 & S & 1.685171 & 2.312172 & -1.227608 \\
\hline $\mathrm{H}$ & -0.879506 & 2.452636 & -1.448742 & 0 & 0.981122 & 1.200388 & -1.949384 \\
\hline C & -3.166701 & 0.255430 & -2.753627 & 0 & 0.859271 & 2.918705 & -0.173679 \\
\hline $\mathrm{H}$ & -3.795185 & -1.588408 & -1.808656 & $\mathrm{C}$ & 1.708015 & 3.601681 & -2.546818 \\
\hline $\mathrm{H}$ & -2.365711 & 2.145030 & -3.434229 & $\mathrm{~F}$ & 2.327653 & 4.693446 & -2.102288 \\
\hline $\mathrm{H}$ & -3.819960 & 0.132293 & -3.611694 & $\mathrm{~F}$ & 2.342615 & 3.160898 & -3.629848 \\
\hline C & -2.477959 & -0.043010 & 2.936947 & $\mathrm{~F}$ & 0.462005 & 3.942587 & -2.897459 \\
\hline C & -1.232665 & -0.442625 & 3.473153 & $\mathrm{C}$ & -2.208959 & 1.221943 & -0.469327 \\
\hline C & -0.104218 & 0.260114 & 3.056193 & C & -2.311695 & -0.073230 & -0.986102 \\
\hline C & -0.184703 & 1.348306 & 2.179256 & C & -2.059236 & 2.314150 & -1.320838 \\
\hline C & -1.430395 & 1.673380 & 1.591391 & C & -2.263613 & -0.267557 & -2.367350 \\
\hline C & -2.585822 & 0.977029 & 2.015410 & $\mathrm{H}$ & -2.415749 & -0.928261 & -0.320094 \\
\hline $\mathrm{H}$ & -3.364190 & -0.588558 & 3.247903 & $\mathrm{C}$ & -2.027085 & 2.111300 & -2.698767 \\
\hline $\mathrm{H}$ & 0.683080 & 1.989496 & 2.032979 & $\mathrm{H}$ & -1.933274 & 3.314427 & -0.915770 \\
\hline $\mathrm{H}$ & -1.486858 & 2.577586 & 0.992463 & $\mathrm{C}$ & -2.126598 & 0.824647 & -3.222912 \\
\hline $\mathrm{H}$ & -3.551298 & 1.238680 & 1.593316 & $\mathrm{H}$ & -2.331800 & -1.276359 & -2.764258 \\
\hline 0 & 1.120853 & -0.127195 & 3.561575 & $\mathrm{H}$ & -1.898309 & 2.961130 & -3.360678 \\
\hline C & 2.121623 & -0.394185 & 2.676347 & $\mathrm{H}$ & -2.083045 & 0.671576 & -4.296737 \\
\hline 0 & 2.031861 & -0.179789 & 1.488075 & C & -3.015354 & -0.304468 & 2.645946 \\
\hline C & 3.309080 & -0.989483 & 3.352124 & C & -1.672084 & -0.574984 & 3.043377 \\
\hline $\mathrm{H}$ & 3.086867 & -2.050804 & 3.507990 & C & -0.608694 & 0.199586 & 2.509179 \\
\hline $\mathrm{H}$ & 4.179583 & -0.890820 & 2.705095 & C & -0.843918 & 1.177449 & 1.587953 \\
\hline $\mathrm{H}$ & 3.479671 & -0.518172 & 4.321444 & $\mathrm{C}$ & -2.204969 & 1.422908 & 1.066098 \\
\hline C & -1.117950 & -1.566859 & 4.456040 & C & -3.278791 & 0.635972 & 1.705504 \\
\hline $\mathrm{H}$ & -0.745816 & -1.193664 & 5.416711 & $\mathrm{H}$ & -3.807096 & -0.906371 & 3.079490 \\
\hline $\mathrm{H}$ & -2.087742 & -2.043624 & 4.608442 & $\mathrm{H}$ & -0.042071 & 1.792313 & 1.176681 \\
\hline \multirow[t]{2}{*}{$\mathrm{H}$} & -0.408489 & -2.314164 & 4.088885 & $\mathrm{H}$ & -2.420747 & 2.495450 & 1.221252 \\
\hline & & & & $\mathrm{H}$ & -4.295053 & 0.807040 & 1.358507 \\
\hline$===$ Int 2 & $===$ & & & 0 & 0.638006 & -0.078253 & 3.013885 \\
\hline $\mathrm{Cu}$ & 0.482862 & -0.498647 & -1.086449 & $\mathrm{C}$ & 1.685227 & -0.250803 & 2.132249 \\
\hline O & 0.030034 & -2.391072 & -0.642101 & 0 & 1.581661 & -0.008787 & 0.960934 \\
\hline O & 3.080571 & 2.058279 & -0.918659 & $\mathrm{C}$ & 2.895425 & -0.768258 & 2.833670 \\
\hline S & -0.367898 & -2.912882 & 0.694610 & $\mathrm{H}$ & 2.756055 & -1.847644 & 2.957074 \\
\hline 0 & 0.638263 & -2.749108 & 1.747095 & $\mathrm{H}$ & 3.771818 & -0.580260 & 2.214355 \\
\hline 0 & -1.736422 & -2.554188 & 1.089217 & $\mathrm{H}$ & 3.002944 & -0.309248 & 3.818032 \\
\hline
\end{tabular}




\begin{tabular}{|c|c|c|c|c|c|c|c|}
\hline C & -1.369184 & -1.627986 & 4.039287 & $\mathrm{C}$ & -3.144081 & 0.781735 & 1.939478 \\
\hline $\mathrm{H}$ & -0.914182 & -1.169511 & 4.927182 & $\mathrm{H}$ & -3.890279 & -0.584781 & 3.401047 \\
\hline $\mathrm{H}$ & -2.261659 & -2.186437 & 4.320909 & $\mathrm{H}$ & 0.211419 & 1.318525 & 1.327666 \\
\hline \multirow[t]{2}{*}{$\mathrm{H}$} & -0.614158 & -2.302782 & 3.615808 & $\mathrm{H}$ & -1.877918 & 2.413820 & 1.822584 \\
\hline & & & & $\mathrm{H}$ & -4.122026 & 1.112224 & 1.599066 \\
\hline$===\mathrm{TS} 2$ & $===$ & & & 0 & 0.591408 & -0.662257 & 3.171781 \\
\hline $\mathrm{Cu}$ & 1.201212 & 2.173598 & -0.758038 & C & 1.846632 & -0.167273 & 2.835627 \\
\hline 0 & 2.047705 & 0.515832 & -1.275879 & 0 & 2.010212 & 0.936197 & 2.401763 \\
\hline 0 & 1.524846 & 4.944822 & 1.562960 & C & 2.887653 & -1.201467 & 3.116602 \\
\hline S & 2.028185 & -0.800748 & -0.560700 & $\mathrm{H}$ & 2.782411 & -1.996328 & 2.371883 \\
\hline 0 & 3.307594 & -1.215396 & -0.014079 & $\mathrm{H}$ & 3.872582 & -0.748847 & 3.013184 \\
\hline 0 & 0.855350 & -0.967914 & 0.311170 & $\mathrm{H}$ & 2.748105 & -1.625547 & 4.113958 \\
\hline C & 1.688368 & -1.937153 & -1.972589 & C & -1.599701 & -1.652267 & 4.389156 \\
\hline F & 1.609996 & -3.193193 & -1.538051 & $\mathrm{H}$ & -0.971152 & -1.280606 & 5.206286 \\
\hline F & 0.520730 & -1.613926 & -2.540789 & $\mathrm{H}$ & -2.569960 & -1.958093 & 4.782070 \\
\hline F & 2.644065 & -1.855948 & -2.890280 & $\mathrm{H}$ & -1.077324 & -2.523107 & 3.976162 \\
\hline S & 0.263212 & 4.580028 & 0.951671 & & & & \\
\hline 0 & 0.381738 & 3.873270 & -0.369178 & $===\operatorname{Int} 3$ & $===$ & & \\
\hline 0 & -0.760198 & 3.981123 & 1.823076 & $\mathrm{Cu}$ & 0.144334 & -0.203593 & -1.494158 \\
\hline C & -0.511978 & 6.157706 & 0.392693 & 0 & 0.563242 & -2.089495 & -1.648622 \\
\hline F & -0.725077 & 6.952154 & 1.438326 & 0 & 2.308896 & 2.739439 & -0.700246 \\
\hline F & 0.275545 & 6.786192 & -0.473111 & S & 1.385845 & -2.772282 & -0.587421 \\
\hline F & -1.686066 & 5.913520 & -0.198358 & 0 & 2.782700 & -2.373036 & -0.549996 \\
\hline C & -2.054797 & 1.733764 & -0.151262 & 0 & 0.686195 & -2.835768 & 0.701132 \\
\hline C & -1.471829 & 0.880457 & -1.092109 & C & 1.362140 & -4.504278 & -1.218995 \\
\hline C & -2.681094 & 2.909804 & -0.560360 & $\mathrm{~F}$ & 2.023982 & -5.291059 & -0.373123 \\
\hline C & -1.504558 & 1.220935 & -2.445182 & $\mathrm{~F}$ & 0.108799 & -4.943318 & -1.320447 \\
\hline $\mathrm{H}$ & -0.978796 & -0.034754 & -0.769587 & $\mathrm{~F}$ & 1.939607 & -4.572491 & -2.413866 \\
\hline C & -2.721331 & 3.238201 & -1.912530 & S & 0.929662 & 2.521558 & -0.346907 \\
\hline $\mathrm{H}$ & -3.092256 & 3.593999 & 0.177981 & 0 & 0.083322 & 1.758493 & -1.292812 \\
\hline C & -2.127553 & 2.399078 & -2.854528 & 0 & 0.697252 & 2.065245 & 1.092092 \\
\hline $\mathrm{H}$ & -1.036796 & 0.562649 & -3.171334 & C & 0.076720 & 4.156772 & -0.260824 \\
\hline $\mathrm{H}$ & -3.187139 & 4.167899 & -2.224639 & $\mathrm{~F}$ & 0.695168 & 4.929303 & 0.618307 \\
\hline H & -2.141986 & 2.668207 & -3.906302 & F & 0.123103 & 4.717863 & -1.460369 \\
\hline C & -3.015031 & -0.152216 & 2.926527 & $\mathrm{~F}$ & -1.189084 & 3.994890 & 0.102087 \\
\hline C & -1.734723 & -0.607406 & 3.334429 & C & -2.668178 & 0.175591 & -0.803484 \\
\hline C & -0.564085 & -0.085217 & 2.741401 & C & -2.636571 & -1.103123 & -1.382824 \\
\hline C & -0.671984 & 0.872762 & 1.760390 & C & -3.025200 & 1.264512 & -1.612072 \\
\hline C & -1.965126 & 1.390606 & 1.331988 & $\mathrm{C}$ & -2.950101 & -1.283183 & -2.729591 \\
\hline
\end{tabular}




\begin{tabular}{|c|c|c|c|c|c|c|c|}
\hline $\mathrm{H}$ & -2.376415 & -1.969959 & -0.781149 & $\mathrm{~F}$ & -0.496443 & 1.587211 & -4.553374 \\
\hline C & -3.351115 & 1.081806 & -2.952155 & S & -2.078154 & -2.197131 & 1.494030 \\
\hline $\mathrm{H}$ & -3.008755 & 2.269110 & -1.199896 & 0 & -1.832458 & -1.217689 & 0.385990 \\
\hline C & -3.311503 & -0.192603 & -3.518113 & 0 & -0.860463 & -2.475961 & 2.267625 \\
\hline $\mathrm{H}$ & -2.909752 & -2.280457 & -3.157009 & C & -2.370728 & -3.737312 & 0.523365 \\
\hline $\mathrm{H}$ & -3.616171 & 1.940667 & -3.561346 & $\mathrm{~F}$ & -2.591682 & -4.754704 & 1.352071 \\
\hline $\mathrm{H}$ & -3.553255 & -0.333661 & -4.567104 & $\mathrm{~F}$ & -3.419512 & -3.593429 & -0.276683 \\
\hline C & -2.226780 & 1.688633 & 2.660367 & $\mathrm{~F}$ & -1.294956 & -4.012494 & -0.214895 \\
\hline C & -1.253991 & 0.866013 & 3.237020 & $\mathrm{C}$ & 1.197600 & 0.100326 & 0.301375 \\
\hline C & -0.831588 & -0.205016 & 2.458228 & C & 1.989266 & 0.978358 & -0.385393 \\
\hline C & -1.316407 & -0.475652 & 1.190535 & C & 1.441579 & -1.165122 & 0.752697 \\
\hline C & -2.249811 & 0.387176 & 0.607305 & C & 3.240223 & 0.444392 & -0.726265 \\
\hline C & -2.707728 & 1.466696 & 1.373506 & $\mathrm{H}$ & 1.677703 & 1.981795 & -0.667274 \\
\hline $\mathrm{H}$ & -2.600500 & 2.534881 & 3.231291 & $\mathrm{C}$ & 2.711126 & -1.645311 & 0.379782 \\
\hline $\mathrm{H}$ & -0.926571 & -1.350396 & 0.676994 & $\mathrm{H}$ & 0.734205 & -1.770623 & 1.321499 \\
\hline $\mathrm{H}$ & 1.119487 & 1.074306 & 1.403028 & $\mathrm{C}$ & 3.594436 & -0.848555 & -0.343305 \\
\hline $\mathrm{H}$ & -3.457648 & 2.138811 & 0.967068 & $\mathrm{H}$ & 3.925045 & 1.068512 & -1.292440 \\
\hline O & 0.157048 & -1.065286 & 2.971473 & $\mathrm{H}$ & 2.976969 & -2.658755 & 0.666667 \\
\hline C & 1.382063 & -0.951235 & 2.488853 & $\mathrm{H}$ & 4.570961 & -1.238347 & -0.612527 \\
\hline 0 & 1.742142 & -0.019202 & 1.760408 & C & -2.586365 & 1.015850 & 2.889445 \\
\hline C & 2.338775 & -1.992619 & 2.955490 & C & -1.532344 & 0.347928 & 3.516934 \\
\hline $\mathrm{H}$ & 3.044742 & -2.193454 & 2.147368 & C & -0.234210 & 0.616191 & 3.060983 \\
\hline $\mathrm{H}$ & 2.884571 & -1.594627 & 3.818399 & $\mathrm{C}$ & 0.013798 & 1.591992 & 2.050611 \\
\hline $\mathrm{H}$ & 1.804869 & -2.898866 & 3.236543 & C & -1.090311 & 2.320332 & 1.521454 \\
\hline C & -0.661022 & 1.128174 & 4.593610 & $\mathrm{C}$ & -2.384084 & 1.988344 & 1.896474 \\
\hline $\mathrm{H}$ & 0.392206 & 1.422257 & 4.509219 & $\mathrm{H}$ & -3.599183 & 0.760136 & 3.187475 \\
\hline $\mathrm{H}$ & -1.194234 & 1.938390 & 5.096231 & $\mathrm{H}$ & 1.018376 & 1.960764 & 1.900149 \\
\hline \multirow[t]{2}{*}{$\mathrm{H}$} & -0.704141 & 0.236860 & 5.226790 & $\mathrm{H}$ & -0.888000 & 3.123843 & 0.818477 \\
\hline & & & & $\mathrm{H}$ & -3.230525 & 2.501132 & 1.451911 \\
\hline$===\operatorname{TS} 1$ & (ortho) $===$ & & & 0 & 0.729707 & -0.152453 & 3.636581 \\
\hline $\mathrm{Cu}$ & -0.768414 & 0.459715 & 0.197563 & $\mathrm{C}$ & 2.087860 & 0.047152 & 3.532282 \\
\hline O & -0.969573 & 1.095281 & -1.695011 & 0 & 2.585788 & 1.022514 & 3.044060 \\
\hline O & -3.295767 & -1.952299 & 2.250090 & $\mathrm{C}$ & 2.802714 & -1.122102 & 4.140029 \\
\hline S & -0.811057 & 2.530727 & -2.118728 & $\mathrm{H}$ & 2.417862 & -2.052963 & 3.714701 \\
\hline 0 & -2.049185 & 3.181039 & -2.496457 & $\mathrm{H}$ & 3.871732 & -1.022352 & 3.956612 \\
\hline O & 0.098834 & 3.262386 & -1.223808 & $\mathrm{H}$ & 2.608652 & -1.146350 & 5.216719 \\
\hline C & 0.164096 & 2.327107 & -3.671727 & C & -1.762780 & -0.622114 & 4.640181 \\
\hline $\mathrm{F}$ & 0.423667 & 3.515624 & -4.207680 & $\mathrm{H}$ & -1.312119 & -0.242857 & 5.564231 \\
\hline $\mathrm{F}$ & 1.327952 & 1.726042 & -3.394022 & $\mathrm{H}$ & -2.832584 & -0.767811 & 4.797727 \\
\hline
\end{tabular}




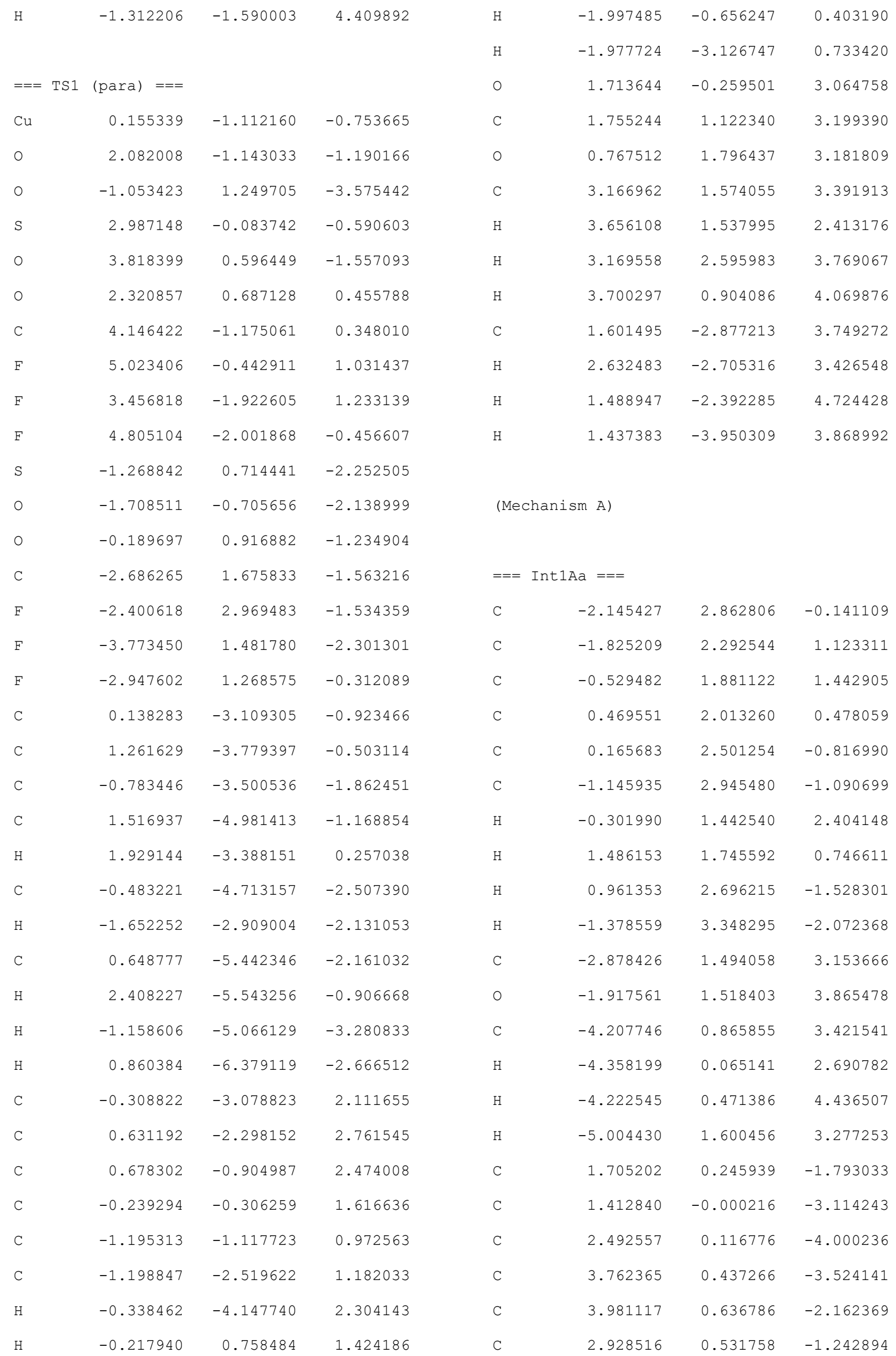




\begin{tabular}{|c|c|c|c|c|c|c|c|}
\hline $\mathrm{H}$ & 0.422071 & -0.281337 & -3.463786 & C & -0.638532 & 2.293150 & 2.130366 \\
\hline $\mathrm{H}$ & 2.323351 & -0.062371 & -5.057590 & 0 & 0.086325 & 1.353274 & 1.784579 \\
\hline $\mathrm{H}$ & 4.592373 & 0.513390 & -4.219074 & $\mathrm{C}$ & -0.922212 & 2.576185 & 3.566230 \\
\hline $\mathrm{H}$ & 4.976222 & 0.859958 & -1.789239 & $\mathrm{H}$ & -1.907020 & 2.146781 & 3.777567 \\
\hline $\mathrm{H}$ & 3.088655 & 0.619313 & -0.174211 & $\mathrm{H}$ & -0.175011 & 2.088021 & 4.190735 \\
\hline $\mathrm{Cu}$ & 0.010856 & 0.189358 & -0.855228 & $\mathrm{H}$ & -0.952684 & 3.651715 & 3.746717 \\
\hline S & -2.834098 & -0.607293 & 0.017089 & $\mathrm{C}$ & 2.120884 & 0.428797 & 0.334236 \\
\hline O & -1.965387 & -0.036078 & -1.078792 & C & 2.972589 & 1.140552 & -0.472161 \\
\hline O & -2.102843 & -0.735486 & 1.276631 & $\mathrm{C}$ & 4.326932 & 1.062643 & -0.125669 \\
\hline O & -4.153797 & 0.007919 & 0.060497 & C & 4.737473 & 0.275986 & 0.949511 \\
\hline C & -3.140686 & -2.330988 & -0.571048 & $\mathrm{C}$ & 3.809983 & -0.446095 & 1.697716 \\
\hline $\mathrm{F}$ & -3.919359 & -2.962264 & 0.303847 & $\mathrm{C}$ & 2.443654 & -0.384206 & 1.392792 \\
\hline $\mathrm{F}$ & -3.759396 & -2.299255 & -1.752140 & $\mathrm{H}$ & 2.631271 & 1.685784 & -1.344172 \\
\hline $\mathrm{F}$ & -2.002659 & -2.997255 & -0.702484 & $\mathrm{H}$ & 5.054262 & 1.600996 & -0.725859 \\
\hline S & 1.138975 & -2.039950 & 0.649125 & $\mathrm{H}$ & 5.792407 & 0.212420 & 1.196392 \\
\hline o & 1.737057 & -0.840357 & 1.237284 & $\mathrm{H}$ & 4.131118 & -1.069807 & 2.526288 \\
\hline O & 0.383712 & -2.952799 & 1.469475 & $\mathrm{H}$ & 1.705213 & -0.947048 & 1.955841 \\
\hline O & 0.441180 & -1.682062 & -0.670452 & $\mathrm{Cu}$ & 0.206274 & 0.433193 & 0.077013 \\
\hline C & 2.552980 & -3.041656 & 0.004062 & S & -2.728042 & -0.327488 & 1.011748 \\
\hline $\mathrm{F}$ & 2.102020 & -4.133774 & -0.599855 & 0 & -1.723425 & -0.072991 & -0.082708 \\
\hline $\mathrm{F}$ & 3.272591 & -2.335302 & -0.872614 & 0 & -2.697359 & 0.712182 & 2.046157 \\
\hline $\mathrm{F}$ & 3.343974 & -3.393869 & 1.012798 & 0 & -4.019267 & -0.743567 & 0.505912 \\
\hline O & -2.908838 & 2.119058 & 1.912204 & $\mathrm{C}$ & -1.998495 & -1.795994 & 1.860079 \\
\hline C & -3.559408 & 3.268792 & -0.429664 & $\mathrm{~F}$ & -2.673901 & -2.067978 & 2.973922 \\
\hline $\mathrm{H}$ & -3.932774 & 3.960298 & 0.331599 & $\mathrm{~F}$ & -1.999717 & -2.860544 & 1.078376 \\
\hline $\mathrm{H}$ & -3.629181 & 3.745593 & -1.409872 & $\mathrm{~F}$ & -0.729216 & -1.505074 & 2.202924 \\
\hline $\mathrm{H}$ & -4.201780 & 2.381049 & -0.414325 & S & 0.569996 & -1.789853 & -1.690201 \\
\hline & & & & 0 & 0.496539 & -2.340218 & -0.344203 \\
\hline$===$ & Int1As $===$ & & & 0 & -0.317290 & -2.231859 & -2.737931 \\
\hline C & -2.195744 & 2.842179 & -0.854519 & 0 & 0.669399 & -0.248478 & -1.614988 \\
\hline C & -1.050131 & 3.040770 & -0.087129 & $\mathrm{C}$ & 2.272741 & -2.180033 & -2.298926 \\
\hline C & 0.219731 & 3.204133 & -0.639757 & $\mathrm{~F}$ & 2.528867 & -1.505832 & -3.417392 \\
\hline C & 0.368034 & 3.124707 & -2.022028 & F & 3.183259 & -1.847166 & -1.383336 \\
\hline C & -0.749108 & 2.885000 & -2.820821 & $\mathrm{~F}$ & 2.368910 & -3.481462 & -2.544403 \\
\hline C & -2.005122 & 2.757003 & -2.239055 & 0 & -1.196465 & 3.159528 & 1.306650 \\
\hline $\mathrm{H}$ & 1.066535 & 3.408269 & 0.011150 & C & -3.575111 & 2.724637 & -0.266823 \\
\hline $\mathrm{H}$ & 1.346312 & 3.258722 & -2.473669 & $\mathrm{H}$ & -3.573411 & 2.845784 & 0.816233 \\
\hline $\mathrm{H}$ & -0.639900 & 2.801097 & -3.897200 & $\mathrm{H}$ & -4.229850 & 3.482762 & -0.709005 \\
\hline $\mathrm{H}$ & -2.873975 & 2.573670 & -2.865965 & $\mathrm{H}$ & -4.002363 & 1.742013 & -0.494761 \\
\hline
\end{tabular}




\begin{tabular}{|c|c|c|c|c|c|c|c|}
\hline & & & & 0 & -0.019347 & -1.504760 & 1.306248 \\
\hline$===\mathrm{TS} 1 \mathrm{Aa}$ & $a===$ & & & 0 & -0.192669 & -3.955931 & 0.761762 \\
\hline C & -2.981347 & 2.584105 & -0.580950 & 0 & 0.543223 & -2.255721 & -0.928189 \\
\hline C & -2.647656 & 1.877410 & 0.493730 & $\mathrm{C}$ & 2.189726 & -2.896228 & 1.005829 \\
\hline C & -1.373588 & 1.191235 & 0.783510 & $\mathrm{~F}$ & 2.762999 & -3.850799 & 0.279645 \\
\hline C & -0.218460 & 1.607589 & -0.115534 & $\mathrm{~F}$ & 2.870115 & -1.758770 & 0.822472 \\
\hline C & -0.608648 & 2.471031 & -1.250310 & $\mathrm{~F}$ & 2.268903 & -3.226627 & 2.293360 \\
\hline C & -1.880070 & 2.814708 & -1.528822 & 0 & -3.362525 & 1.844038 & 1.721412 \\
\hline $\mathrm{H}$ & -1.525252 & 0.106979 & 0.861937 & C & -4.339104 & 3.154539 & -0.838808 \\
\hline $\mathrm{H}$ & 0.655516 & 1.937219 & 0.443314 & $\mathrm{H}$ & -4.974417 & 3.117765 & 0.048327 \\
\hline $\mathrm{H}$ & 0.195748 & 2.794240 & -1.903696 & $\mathrm{H}$ & -4.265132 & 4.187805 & -1.190944 \\
\hline $\mathrm{H}$ & -2.110383 & 3.387812 & -2.421850 & $\mathrm{H}$ & -4.812691 & 2.549200 & -1.621291 \\
\hline C & -2.477014 & 1.703857 & 2.649178 & & & & \\
\hline o & -1.269131 & 1.525416 & 2.264932 & $===\mathrm{TS} 1 \mathrm{As}$ & $S===$ & & \\
\hline C & -2.862532 & 1.742612 & 4.072906 & $\mathrm{C}$ & -2.903634 & 2.818186 & -0.328687 \\
\hline $\mathrm{H}$ & -3.252978 & 0.751502 & 4.334491 & C & -1.930297 & 2.525072 & 0.564937 \\
\hline $\mathrm{H}$ & -1.988570 & 1.954672 & 4.687978 & $\mathrm{C}$ & -0.523535 & 2.468905 & 0.247477 \\
\hline $\mathrm{H}$ & -3.652571 & 2.479509 & 4.226145 & $\mathrm{C}$ & -0.187749 & 2.283093 & -1.158048 \\
\hline C & 1.642603 & 0.567529 & -1.348925 & $\mathrm{C}$ & -1.248012 & 2.623832 & -2.113756 \\
\hline C & 1.680395 & 0.521164 & -2.738092 & $\mathrm{C}$ & -2.496276 & 2.956916 & -1.717673 \\
\hline C & 2.895833 & 0.775604 & -3.379630 & $\mathrm{H}$ & 0.179986 & 3.016630 & 0.873010 \\
\hline C & 4.029845 & 1.090837 & -2.632646 & $\mathrm{H}$ & 0.799787 & 2.635186 & -1.433740 \\
\hline C & 3.959123 & 1.143172 & -1.241271 & $\mathrm{H}$ & -0.991343 & 2.603943 & -3.168922 \\
\hline C & 2.753769 & 0.881858 & -0.583663 & $\mathrm{H}$ & -3.259326 & 3.217205 & -2.444389 \\
\hline $\mathrm{H}$ & 0.795053 & 0.277059 & -3.322781 & C & -1.292112 & 1.074895 & 2.110224 \\
\hline $\mathrm{H}$ & 2.947991 & 0.723182 & -4.463425 & 0 & -0.277071 & 1.029378 & 1.397994 \\
\hline $\mathrm{H}$ & 4.970182 & 1.294745 & -3.136026 & $\mathrm{C}$ & -1.533711 & 0.175745 & 3.260608 \\
\hline $\mathrm{H}$ & 4.843218 & 1.385240 & -0.658426 & $\mathrm{H}$ & -2.604392 & 0.021576 & 3.392972 \\
\hline $\mathrm{H}$ & 2.707035 & 0.905040 & 0.501761 & $\mathrm{H}$ & -1.021167 & -0.765642 & 3.047817 \\
\hline $\mathrm{Cu}$ & 0.007817 & -0.278052 & -0.798443 & $\mathrm{H}$ & -1.106309 & 0.634679 & 4.159181 \\
\hline S & -3.333587 & -0.785422 & -1.067958 & $\mathrm{C}$ & 1.679142 & 0.475737 & -1.165237 \\
\hline 0 & -1.937805 & -0.409370 & -1.467870 & C & 2.204855 & 0.338826 & -2.437600 \\
\hline 0 & -3.512782 & -0.815790 & 0.388364 & $\mathrm{C}$ & 3.597769 & 0.294518 & -2.553094 \\
\hline O & -4.346700 & -0.085391 & -1.846256 & $\mathrm{C}$ & 4.400354 & 0.408107 & -1.419332 \\
\hline C & -3.419503 & -2.552452 & -1.585628 & $\mathrm{C}$ & 3.821210 & 0.554011 & -0.160887 \\
\hline $\mathrm{F}$ & -4.616521 & -3.055487 & -1.288958 & C & 2.429930 & 0.591792 & -0.012776 \\
\hline $\mathrm{F}$ & -3.225680 & -2.655320 & -2.899305 & $\mathrm{H}$ & 1.575469 & 0.245770 & -3.318872 \\
\hline $\mathrm{F}$ & -2.485856 & -3.252026 & -0.953432 & $\mathrm{H}$ & 4.044357 & 0.167415 & -3.534790 \\
\hline S & 0.425132 & -2.674378 & 0.508253 & $\mathrm{H}$ & 5.480809 & 0.371336 & -1.516881 \\
\hline
\end{tabular}




\begin{tabular}{|c|c|c|c|c|c|c|c|}
\hline $\mathrm{H}$ & 4.445150 & 0.616134 & 0.725954 & $\mathrm{H}$ & -1.106811 & -0.086691 & 4.127427 \\
\hline $\mathrm{H}$ & 1.972013 & 0.642221 & 0.967947 & $\mathrm{H}$ & 0.160256 & 1.165555 & 4.154151 \\
\hline $\mathrm{Cu}$ & -0.224453 & 0.169650 & -1.114179 & $\mathrm{H}$ & -1.550806 & 1.593428 & 4.540322 \\
\hline S & -3.095243 & -0.593392 & -0.532805 & C & 0.982629 & 1.581768 & -1.305571 \\
\hline 0 & -2.161767 & -0.048768 & -1.582532 & C & 0.898372 & 0.878788 & -2.510721 \\
\hline 0 & -2.387915 & -0.822083 & 0.732948 & $\mathrm{C}$ & 1.979749 & 0.112815 & -2.956933 \\
\hline 0 & -4.361829 & 0.121298 & -0.466552 & C & 3.153390 & 0.053374 & -2.206306 \\
\hline C & -3.534499 & -2.261566 & -1.196616 & C & 3.248228 & 0.771613 & -1.014437 \\
\hline F & -4.273324 & -2.908672 & -0.299709 & $\mathrm{C}$ & 2.169228 & 1.529202 & -0.568212 \\
\hline $\mathrm{F}$ & -4.249909 & -2.108140 & -2.312499 & $\mathrm{H}$ & -0.009873 & 0.913195 & -3.108196 \\
\hline $\mathrm{F}$ & -2.453914 & -2.967427 & -1.473959 & $\mathrm{H}$ & 1.891768 & -0.434439 & -3.891564 \\
\hline S & 0.350463 & -2.479352 & 0.344436 & $\mathrm{H}$ & 3.990411 & -0.547731 & -2.548377 \\
\hline O & 0.717664 & -1.569967 & 1.427674 & $\mathrm{H}$ & 4.158252 & 0.730051 & -0.423679 \\
\hline 0 & -0.573542 & -3.558297 & 0.621325 & $\mathrm{H}$ & 2.241057 & 2.064651 & 0.376325 \\
\hline O & 0.053918 & -1.749679 & -0.953621 & $\mathrm{Cu}$ & 0.150025 & -1.647902 & -1.474742 \\
\hline C & 1.928586 & -3.316881 & -0.126832 & S & -2.160218 & -0.862310 & -2.961680 \\
\hline $\mathrm{F}$ & 1.734992 & -4.136695 & -1.156964 & 0 & -0.961776 & -1.759724 & -3.110881 \\
\hline F & 2.854515 & -2.417429 & -0.472402 & 0 & -2.394753 & -0.506800 & -1.555573 \\
\hline $\mathrm{F}$ & 2.393503 & -4.024081 & 0.901917 & 0 & -2.219390 & 0.216955 & -3.938197 \\
\hline 0 & -2.185040 & 2.035803 & 1.850968 & $\mathrm{C}$ & -3.546947 & -1.996731 & -3.397863 \\
\hline C & -4.340455 & 2.985958 & 0.057505 & $\mathrm{~F}$ & -4.706847 & -1.347916 & -3.283231 \\
\hline $\mathrm{H}$ & -4.539990 & 2.571431 & 1.046507 & $\mathrm{~F}$ & -3.420119 & -2.427150 & -4.649644 \\
\hline $\mathrm{H}$ & -4.586694 & 4.055060 & 0.059492 & $\mathrm{~F}$ & -3.562009 & -3.045436 & -2.579625 \\
\hline $\mathrm{H}$ & -4.982091 & 2.476970 & -0.663823 & $\mathrm{~S}$ & 0.249074 & -2.058606 & 1.535707 \\
\hline & & & & 0 & -0.903234 & -1.307966 & 2.057153 \\
\hline$===$ & Int $2 \mathrm{Aa}===$ & & & 0 & 0.130380 & -3.499352 & 1.456259 \\
\hline C & -3.154610 & 2.242109 & -0.091355 & 0 & 0.861878 & -1.403237 & 0.331483 \\
\hline C & -2.251595 & 1.666300 & 0.687986 & $\mathrm{C}$ & 1.571925 & -1.721743 & 2.778172 \\
\hline C & -0.863826 & 1.314359 & 0.304940 & $\mathrm{~F}$ & 2.711207 & -2.313327 & 2.446987 \\
\hline C & -0.235178 & 2.260001 & -0.703791 & $\mathrm{~F}$ & 1.789385 & -0.401055 & 2.875764 \\
\hline C & -1.316463 & 2.648015 & -1.701983 & $\mathrm{~F}$ & 1.188062 & -2.159578 & 3.979098 \\
\hline C & -2.625792 & 2.619618 & -1.422933 & 0 & -2.358785 & 1.339946 & 2.061755 \\
\hline $\mathrm{H}$ & -0.793456 & 0.268610 & -0.009377 & $\mathrm{C}$ & -4.579369 & 2.520371 & 0.269965 \\
\hline $\mathrm{H}$ & 0.082391 & 3.174025 & -0.173125 & $\mathrm{H}$ & -4.792806 & 2.269684 & 1.311114 \\
\hline $\mathrm{H}$ & -0.979962 & 2.990498 & -2.676385 & $\mathrm{H}$ & -4.817216 & 3.575514 & 0.100986 \\
\hline $\mathrm{H}$ & -3.349015 & 2.913556 & -2.178762 & $\mathrm{H}$ & -5.237200 & 1.924165 & -0.371887 \\
\hline C & -1.149914 & 1.203417 & 2.505677 & & & & \\
\hline 0 & -0.219070 & 1.352066 & 1.640234 & \multicolumn{4}{|c|}{$===\operatorname{Int} 2 \mathrm{AS}===$} \\
\hline C & -0.888923 & 0.970189 & 3.935947 & $\mathrm{C}$ & -2.502387 & 3.011009 & -0.397036 \\
\hline
\end{tabular}




\begin{tabular}{|c|c|c|c|c|c|c|c|}
\hline C & -1.933670 & 2.193984 & 0.477939 & $\mathrm{C}$ & 3.123102 & -3.135122 & 2.071515 \\
\hline C & -0.536982 & 2.302944 & 0.980668 & $\mathrm{~F}$ & 3.340733 & -4.442106 & 1.967148 \\
\hline C & 0.450086 & 2.780236 & -0.087183 & $\mathrm{~F}$ & 3.983170 & -2.494565 & 1.272168 \\
\hline C & -0.238448 & 3.946329 & -0.783950 & $\mathrm{~F}$ & 3.358499 & -2.758788 & 3.327518 \\
\hline C & -1.571750 & 4.048148 & -0.903677 & 0 & -2.467067 & 1.031035 & 1.059088 \\
\hline $\mathrm{H}$ & -0.467368 & 2.961648 & 1.857302 & $\mathrm{C}$ & -3.903940 & 2.921596 & -0.909079 \\
\hline $\mathrm{H}$ & 1.331918 & 3.160247 & 0.439359 & $\mathrm{H}$ & -4.431682 & 2.068890 & -0.478340 \\
\hline $\mathrm{H}$ & 0.408908 & 4.678315 & -1.258638 & $\mathrm{H}$ & -4.448800 & 3.843269 & -0.675183 \\
\hline $\mathrm{H}$ & -2.008299 & 4.878593 & -1.453307 & $\mathrm{H}$ & -3.888099 & 2.789273 & -1.994813 \\
\hline $\mathrm{C}$ & -1.470839 & 0.352889 & 1.540563 & & & & \\
\hline O & -0.332694 & 0.948868 & 1.496442 & & & & \\
\hline C & -1.665659 & -0.959639 & 2.173049 & & & & \\
\hline $\mathrm{H}$ & -2.694859 & -1.039465 & 2.525881 & & & & \\
\hline $\mathrm{H}$ & -1.491906 & -1.723779 & 1.406354 & & & & \\
\hline $\mathrm{H}$ & -0.929710 & -1.110019 & 2.963245 & & & & \\
\hline C & 0.927648 & 1.701680 & -1.053203 & & & & \\
\hline C & 0.370914 & 1.538618 & -2.325704 & & & & \\
\hline C & 0.814756 & 0.519613 & -3.164582 & & & & \\
\hline C & 1.830450 & -0.345498 & -2.744753 & & & & \\
\hline C & 2.406418 & -0.179174 & -1.475843 & & & & \\
\hline C & 1.944347 & 0.840230 & -0.633698 & & & & \\
\hline $\mathrm{H}$ & -0.439645 & 2.182995 & -2.651794 & & & & \\
\hline $\mathrm{H}$ & 0.350007 & 0.378359 & -4.134711 & & & & \\
\hline $\mathrm{H}$ & 2.187176 & -1.129441 & -3.406780 & & & & \\
\hline $\mathrm{H}$ & 3.221280 & -0.820790 & -1.149456 & & & & \\
\hline $\mathrm{H}$ & 2.371454 & 0.939705 & 0.361124 & & & & \\
\hline $\mathrm{Cu}$ & 0.607722 & -1.588123 & -0.925782 & & & & \\
\hline S & -2.142454 & -0.735738 & -1.942589 & & & & \\
\hline $\mathrm{O}$ & -1.585276 & -1.532784 & -3.026728 & & & & \\
\hline 0 & -1.232060 & -0.681246 & -0.742308 & & & & \\
\hline $\mathrm{O}$ & -2.741188 & 0.556899 & -2.257345 & & & & \\
\hline C & -3.536466 & -1.734896 & -1.259811 & & & & \\
\hline $\mathrm{F}$ & -4.048797 & -1.119235 & -0.183465 & & & & \\
\hline $\mathrm{F}$ & -4.501411 & -1.882365 & -2.160160 & & & & \\
\hline $\mathrm{F}$ & -3.105126 & -2.936416 & -0.881355 & & & & \\
\hline S & 1.392114 & -2.715972 & 1.595099 & & & & \\
\hline 0 & 1.332032 & -1.247526 & 1.698613 & & & & \\
\hline O & 0.539568 & -3.457877 & 2.512676 & & & & \\
\hline 0 & 1.343652 & -3.166175 & 0.170061 & & & & \\
\hline
\end{tabular}




\section{References}

1. Zhao, X. D.; Yeung, C. S.; Dong, V. M. Palladium-Catalyzed Ortho-Arylation of OPhenylcarbamates with Simple Arenes and Sodium Persulfate. J. Am. Chem. Soc. 2010, 132, 58375844 .

2. Murashige, R.; Hayashi, Y.; Ohmori, S.; Torii, A.; Aizu, Y.; Muto, Y.; Murai, Y.; Oda, Y.; Hashimoto, M. Comparisons of O-Acylation and Friedel-Crafts Acylation of Phenols and Acyl Chlorides and Fries Rearrangement of Phenyl Esters in Trifluoromethanesulfonic Acid: Effective Synthesis of Optically Active Homotyrosines. Tetrahedron 2011, 67, 641-649

3. Quasdorf, K. W.; Tian, X.; Garg, N. K. Cross-Coupling Reactions of Aryl Pivalates with Boronic Acids. J. Am. Chem. Soc. 2008, 130, 14422-3

4. Nussbaumer, P.; Bilban, M. Facile Intramolecular O-->C Ester Migration in Benzylphosphonium Salts. J. Org. Chem. 2000, 65, 7660-2

5. Sano, T. Remarkably Fast Acylation of Alcohols with Benzoyl Chloride Promoted by TMEDA. Synthesis 1999, 7, 1141-1144

6. Sun, X.; Sun, Y.; Zhang, C.; Rao, Y. Room-Temperature Pd-Catalyzed C-H Chlorination by Weak Coordination: One-Pot Synthesis of 2-Chlorophenols with Excellent Regioselectivity. Chem. Commun. (Cambridge, U. K) 2014, 50, 1262-4.

7. Phipps, R. J.; Gaunt, M. J. A Meta-Selective Copper-Catalyzed C-H Bond Arylation. Science 2009, 323, 1593-1597.

8. Kauch, M.; Snieckus, V.; Hoppe, D. Substitution of Hydroxybiaryls via Directed OrthoLithiation of N-Silylated O-Aryl N-Isopropylcarbamates. J. Org. Chem. 2005, 70, 7149-58

9. Tezuka, N.; Shimojo, K.; Hirano, K.; Komagawa, S.; Yoshida, K.; Wang, C.; Miyamoto, K.; Saito, T.; Takita, R.; Uchiyama, M. Direct Hydroxylation and Amination of Arenes via Deprotonative Cupration. J. Am. Chem. Soc. 2016, 138, 9166-71.

10. Kikushima, K.; Nishina, Y. Copper-Catalyzed Oxidative Aromatization of 2-Cyclohexen-1Ones to Phenols in the Presence of Catalytic Hydrogen Bromide under Molecular Oxygen. RSC Adv., 2013, 3, 20150-20156.

11.Luo, Q. L.; Tan, J. P.; Li, Z. F.; Nan, W. H.; Xiao, D. R., Suzuki-Miyaura Coupling of Aryl Iodides, Bromides, and Chlorides Catalyzed by Bis(Thiazole) Pincer Palladium Complexes. $J$. Org. Chem. 2012, 77, 8332-7.

12. Mesganaw, T.; Nathel, N. F. F.; Garg, N. K. Cine Substitution of Arenes Using the Aryl Carbamate as a Removable Directing Group. Org. Lett. 2012, 14, 2918-2921.

13. Quasdorf, K. W.; Riener, M.; Petrova, K. V.; Garg, N. K. Suzuki-Miyaura Coupling of Aryl Carbamates, Carbonates, and Sulfamates. J. Am. Chem. Soc. 2009, 131, 17748-17749.

14.Gaussian 09, Revision D.01, Frisch, M. J.; Trucks, G. W.; Schlegel, H. B.; Scuseria, G. E.; Robb, M. A.; Cheeseman, J. R.; Scalmani, G.; Barone, V.; Mennucci, B.; Petersson, G. A.; Nakatsuji, H.; Caricato, M.; Li, X.; Hratchian, H. P.; Izmaylov, A. F.; Bloino, J.; Zheng, G.; Sonnenberg, J. L.; Hada, M.; Ehara, M.; Toyota, K.; Fukuda, R.; Hasegawa, J.; Ishida, M.; Nakajima, T.; Honda, Y.; Kitao, O.; Nakai, H.; Vreven, T.; Montgomery, J. A., Jr.; Peralta, J. E.; Ogliaro, F.; Bearpark, M.; Heyd, J. J.; Brothers, E.; Kudin, K. N.; Staroverov, V. N.; Kobayashi, R.; Normand, J.; Raghavachari, K.; Rendell, A.; Burant, J. C.; Iyengar, S. S.; Tomasi, J.; Cossi, M.; Rega, N.; Millam, J. M.; Klene, M.; Knox, J. E.; Cross, J. B.; Bakken, V.; Adamo, C.; Jaramillo, J.; Gomperts, R.; Stratmann, R. E.; Yazyev, O.; Austin, A. J.; Cammi, R.; Pomelli, C.; 
Ochterski, J. W.; Martin, R. L.; Morokuma, K.; Zakrzewski, V. G.; Voth, G. A.; Salvador, P.; Dannenberg, J. J.; Dapprich, S.; Daniels, A. D.; Farkas, Ö.; Foresman, J. B.; Ortiz, J. V.; Cioslowski, J.; Fox, D. J. Gaussian, Inc., Wallingford CT, 2009.

15. Zhao, Y.; Truhlar, D. G. The M06 Suite of Density Functionals for Main Group Thermochemistry, Thermochemical Kinetics, Noncovalent Interactions, Excited States, and Transition Elements: Two New Functionals and Systematic Testing of Four M06-Class Functionals and 12 Other Functionals. Theor. Chem. Acc. 2008, 120, 215-241.

16. Hehre, W.; Radom, L.; Schleyer, P. v. R.; Pople, J. Ab Initio Molecular Orbital Theory; John Wiley \& Sons: New York, 1986.

17. (a) Hay, P. J.; Wadt, W. R. Ab Initio Effective Core Potentials for Molecular Calculations. Potentials for the Transition Metal Atoms Sc to Hg. J. Chem. Phys. 1985, 82, 270-283. (b) Wadt, W. R.; Hay, P. J. Ab Initio Effective Core Potentials for Molecular Calculations. Potentials for Main Group Elements Na to Bi. J. Chem. Phys. 1985, 82, 284-298. (c) Hay, P. J.; Wadt, W. R. Ab Initio Effective Core Potentials for Molecular Calculations. Potentials for K to Au Including the Outermost Core Orbitals. J. Chem. Phys. 1985, 82, 299-310.

18. Weigend, F.; Ahlrichs, R. Balanced Basis Sets of Split Valence, Triple Zeta Valence and Quadruple Zeta Valence Quality for H to Rn: Design And Assessment of Accuracy. Phys. Chem. Chem. Phys. 2005, 7, 3297-3305. 


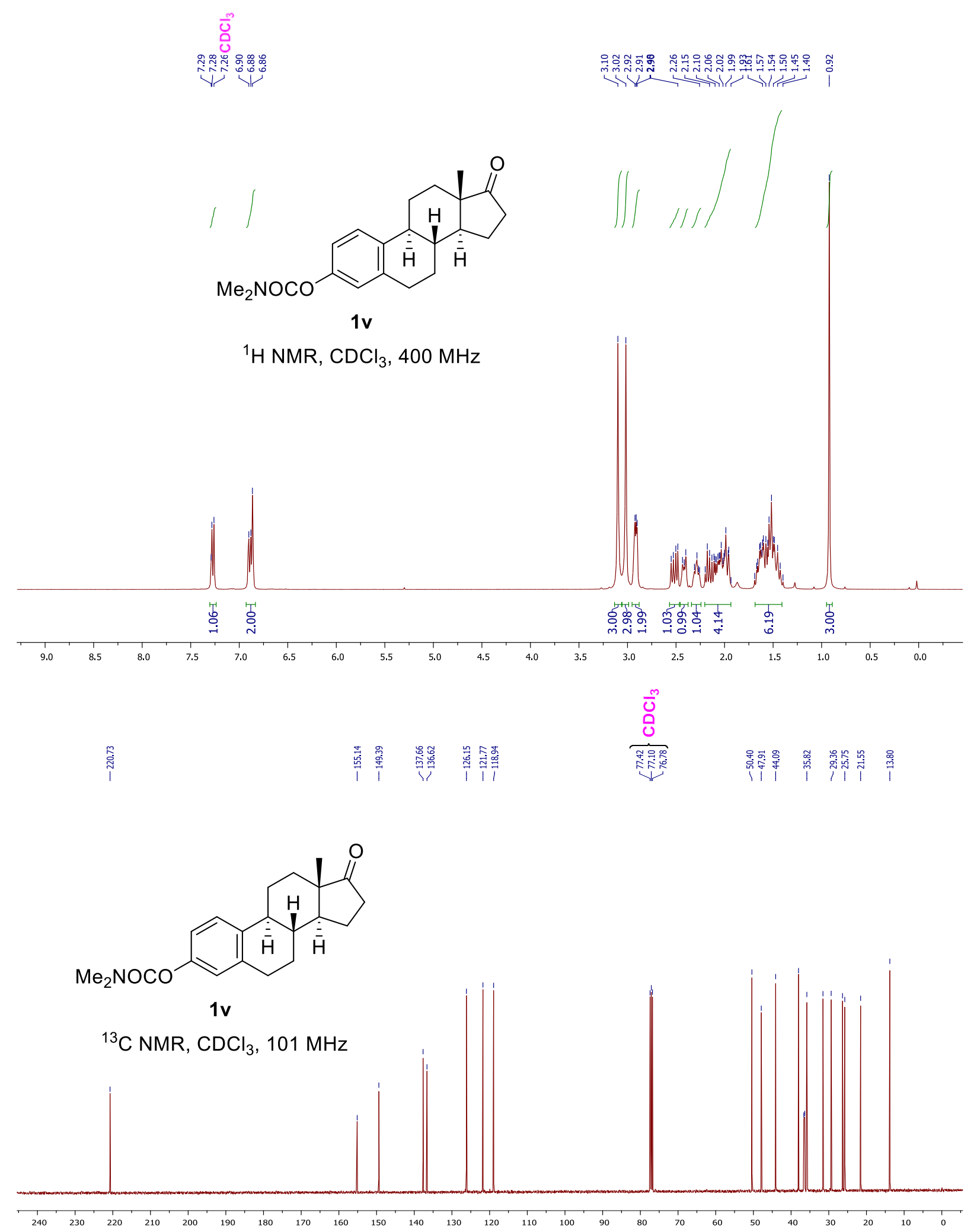




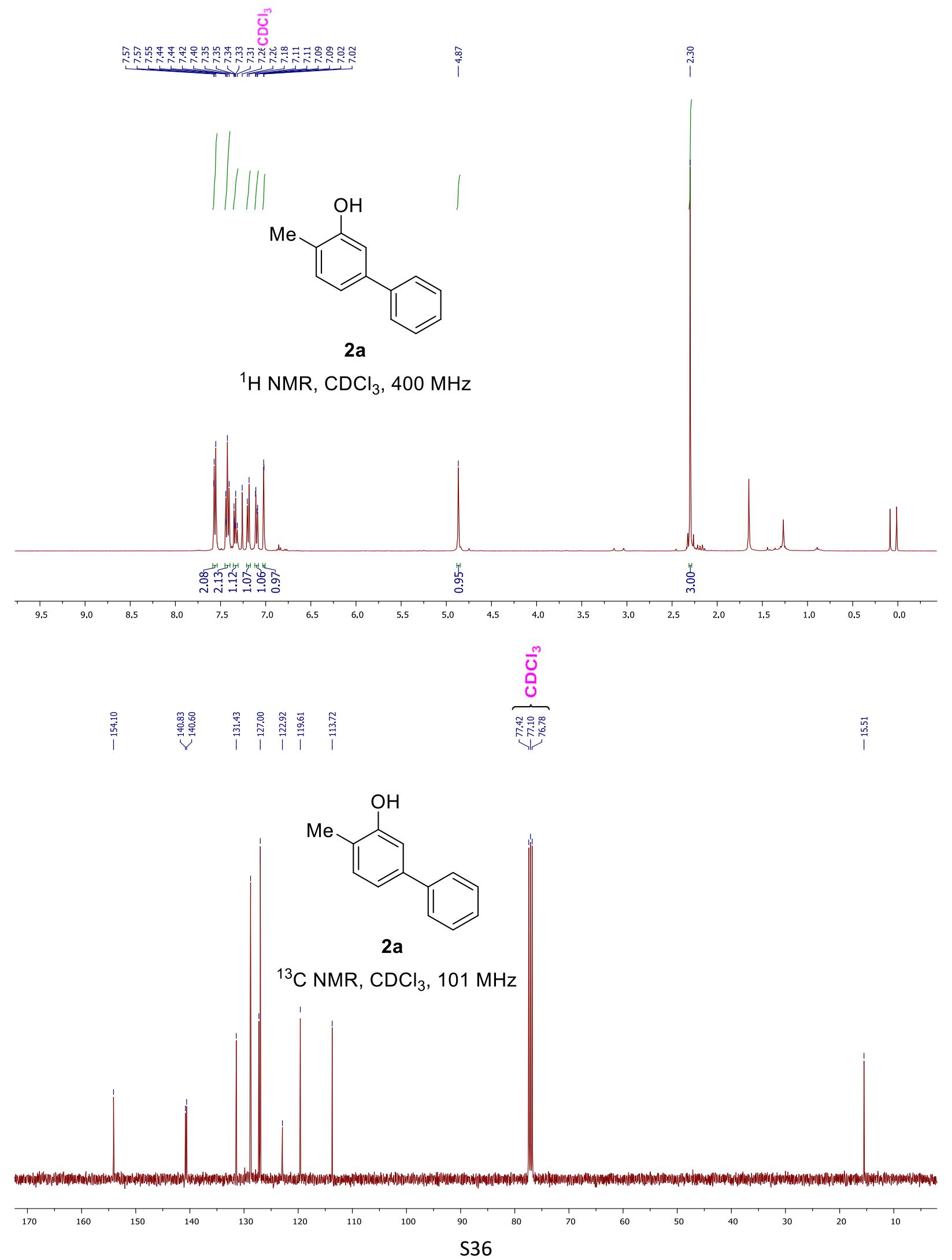




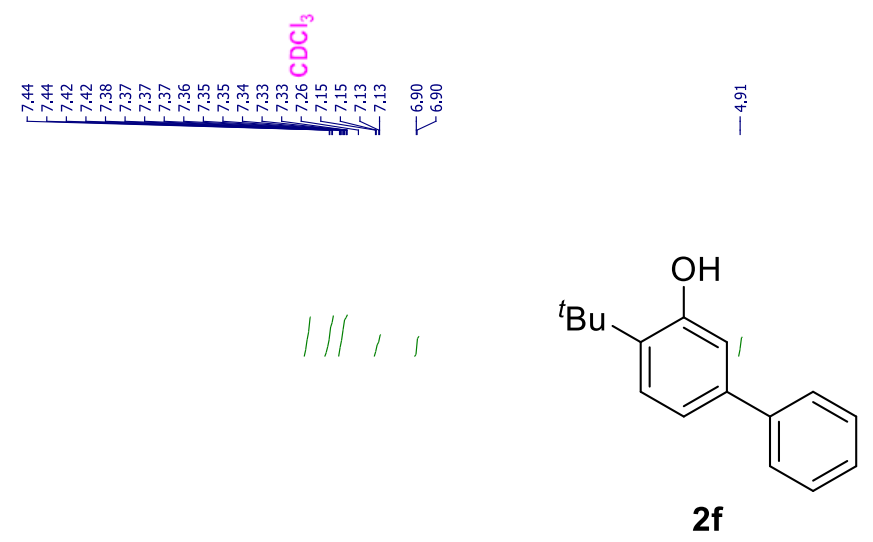

${ }^{1} \mathrm{H}$ NMR, $\mathrm{CDCl}_{3}, 400 \mathrm{MHz}$
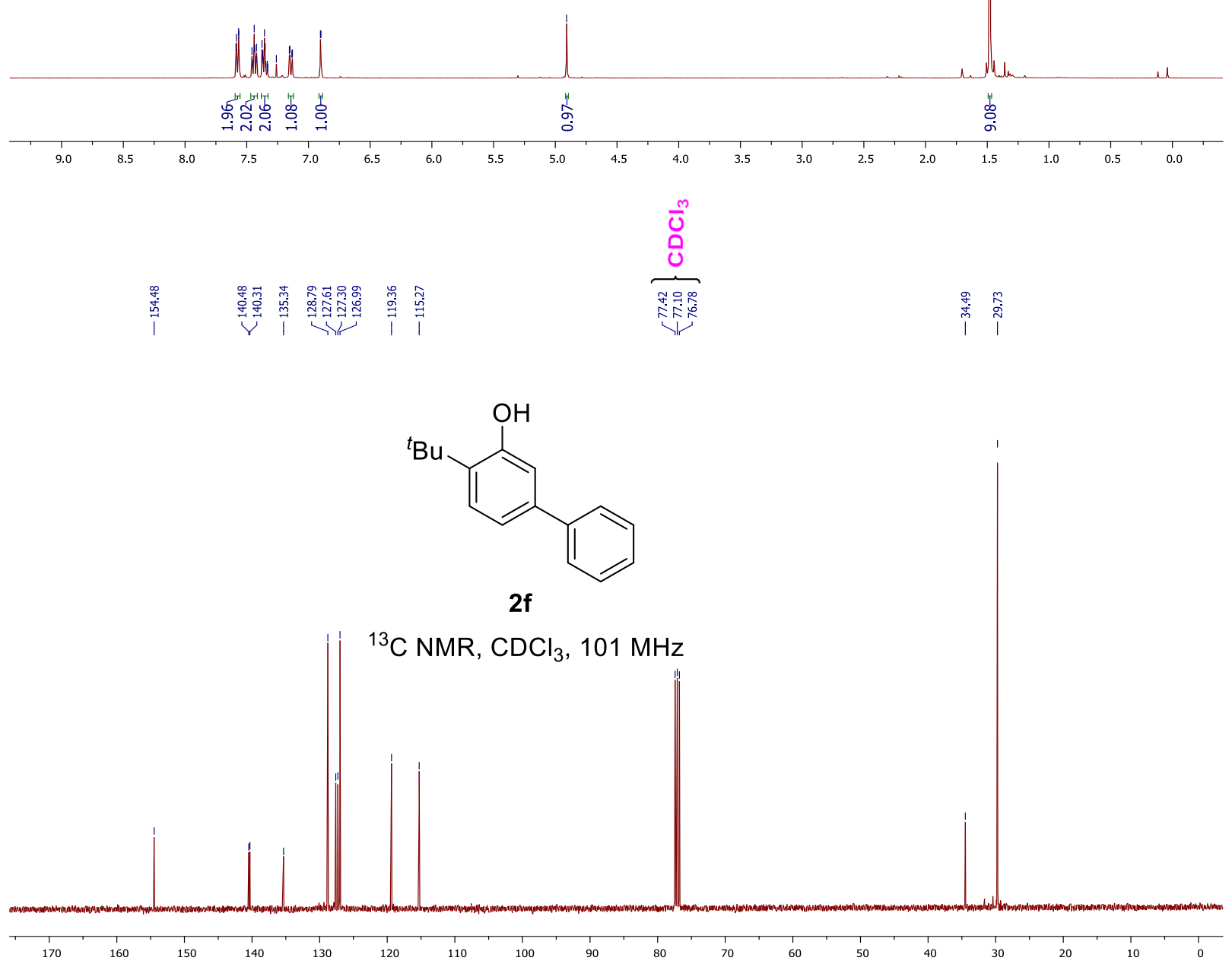

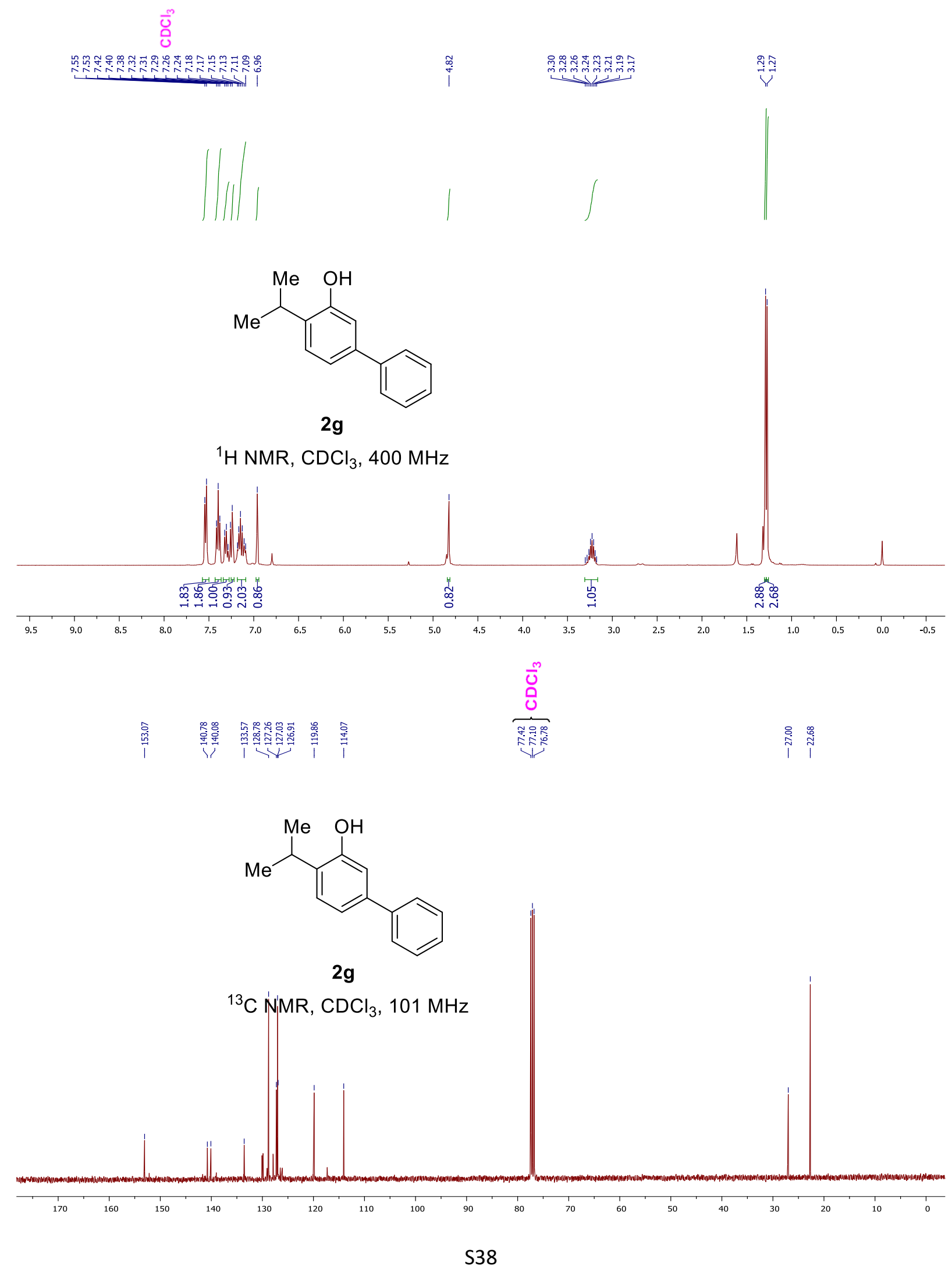


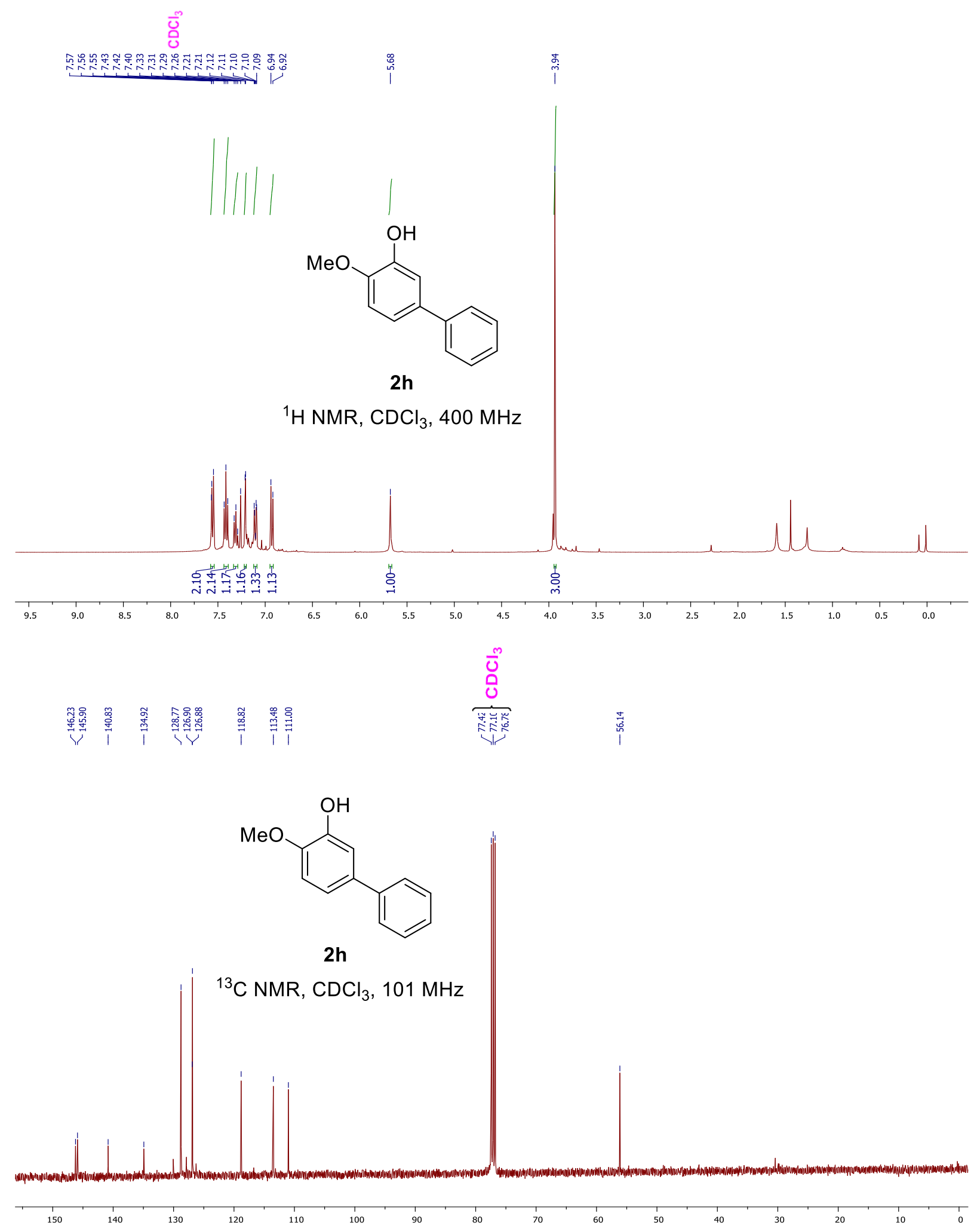




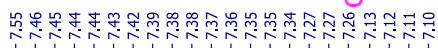

:
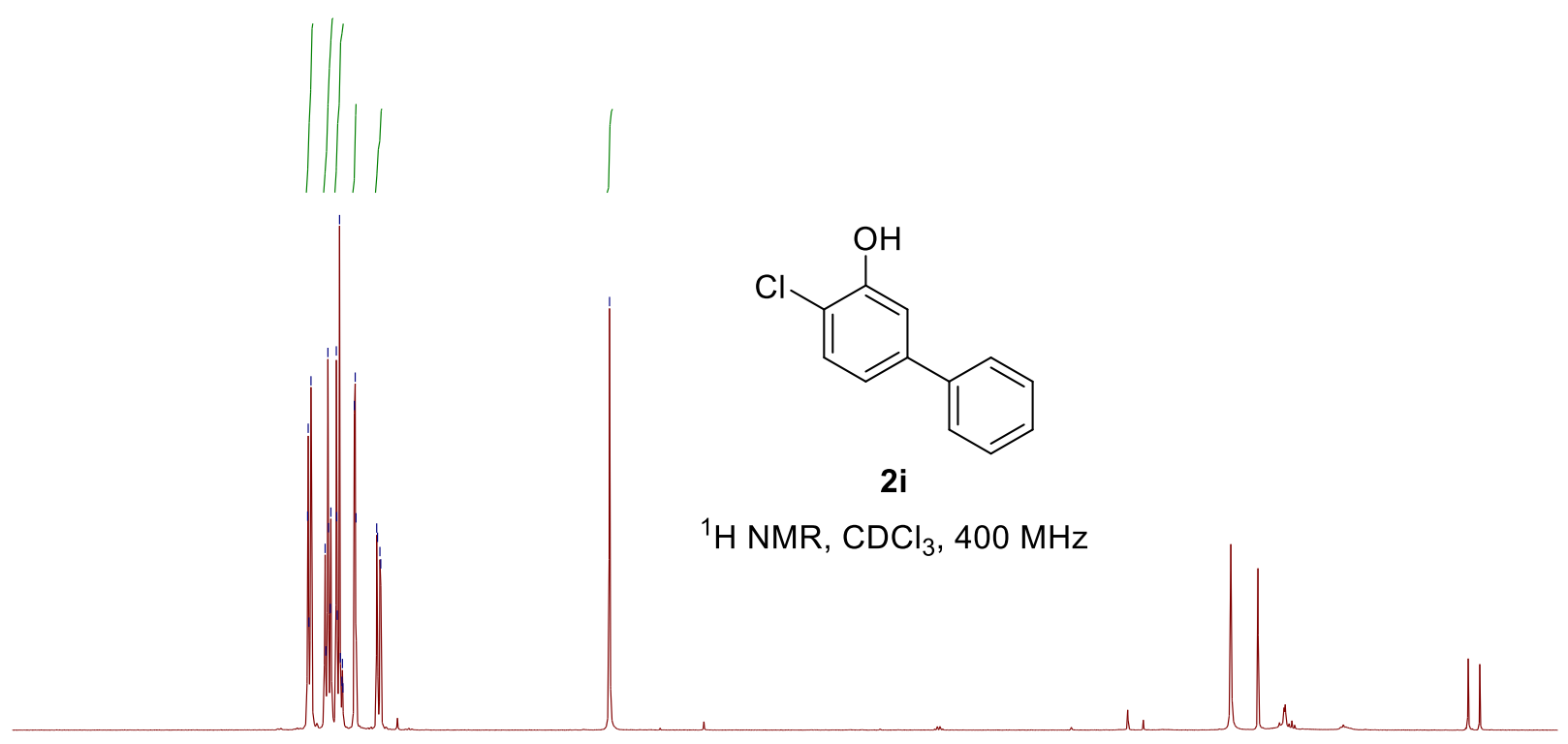

2i
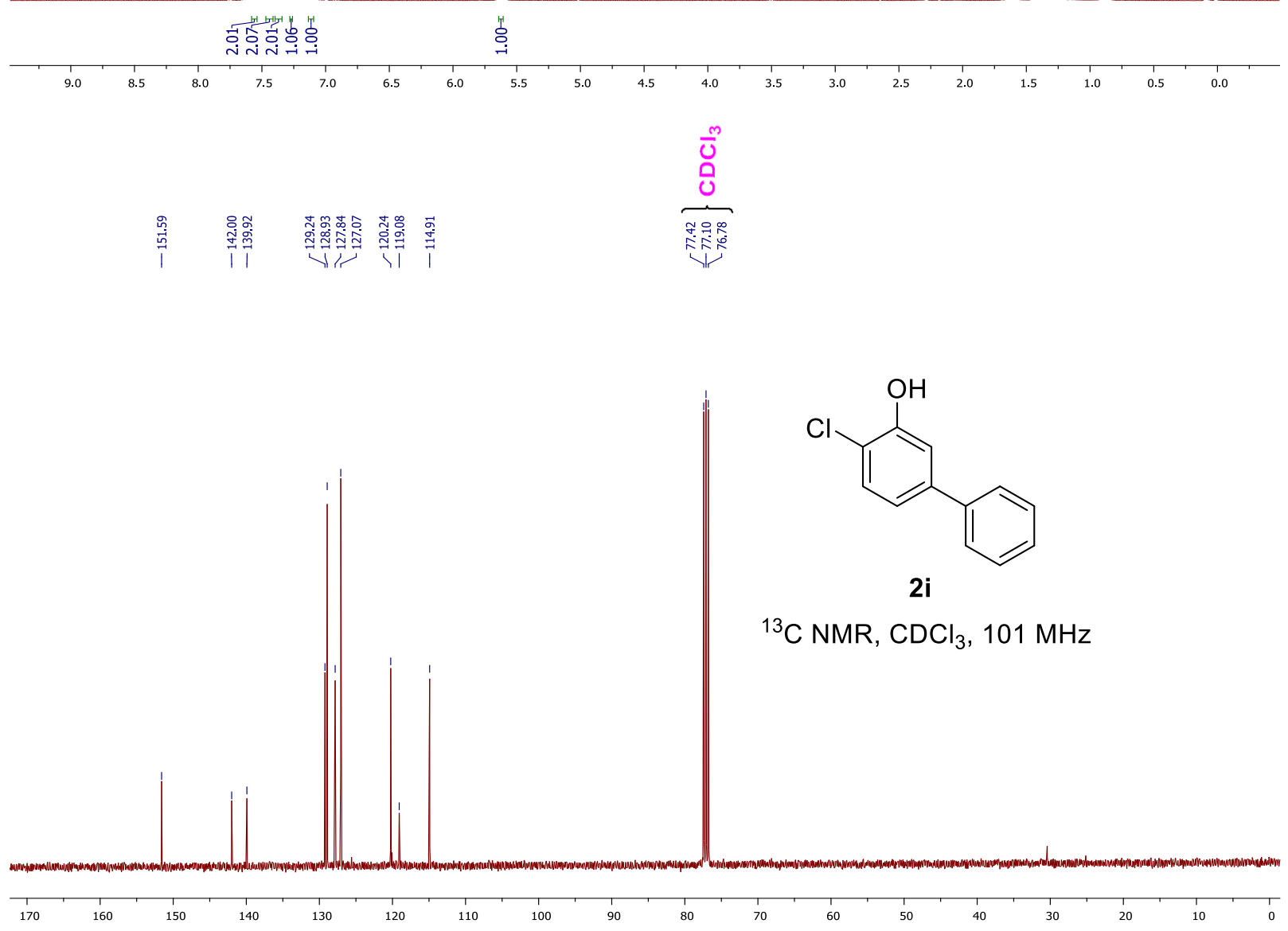

S40 

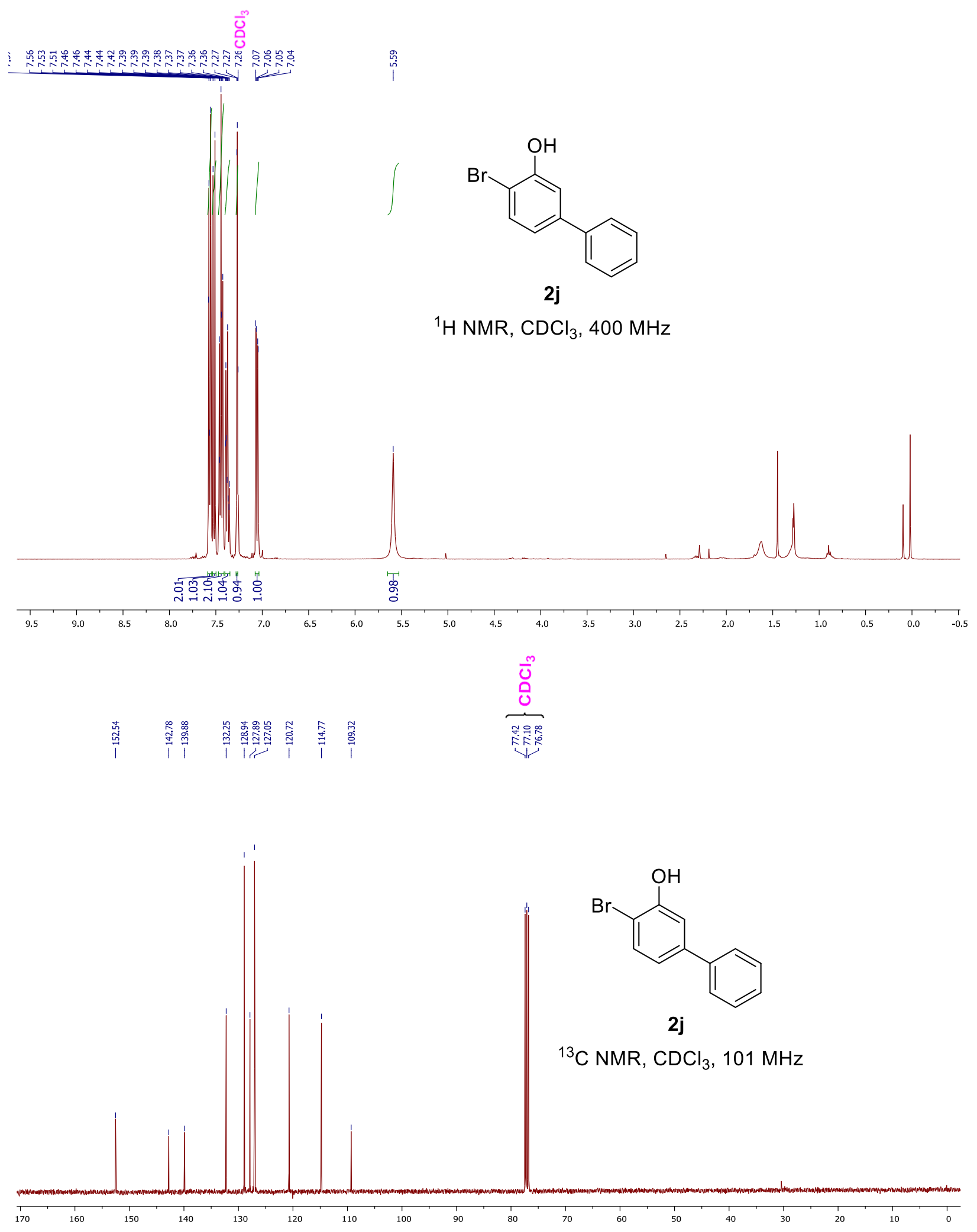

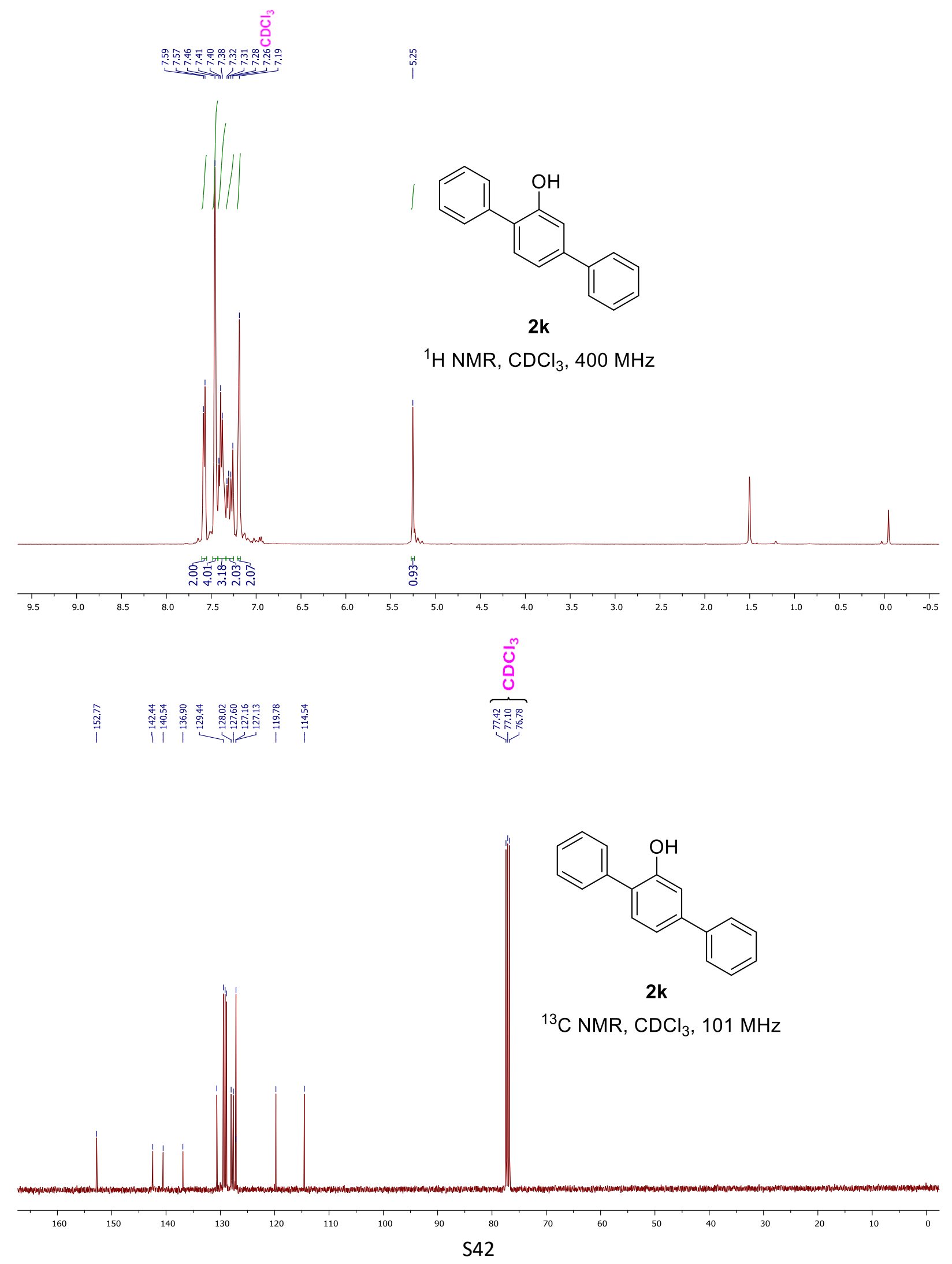

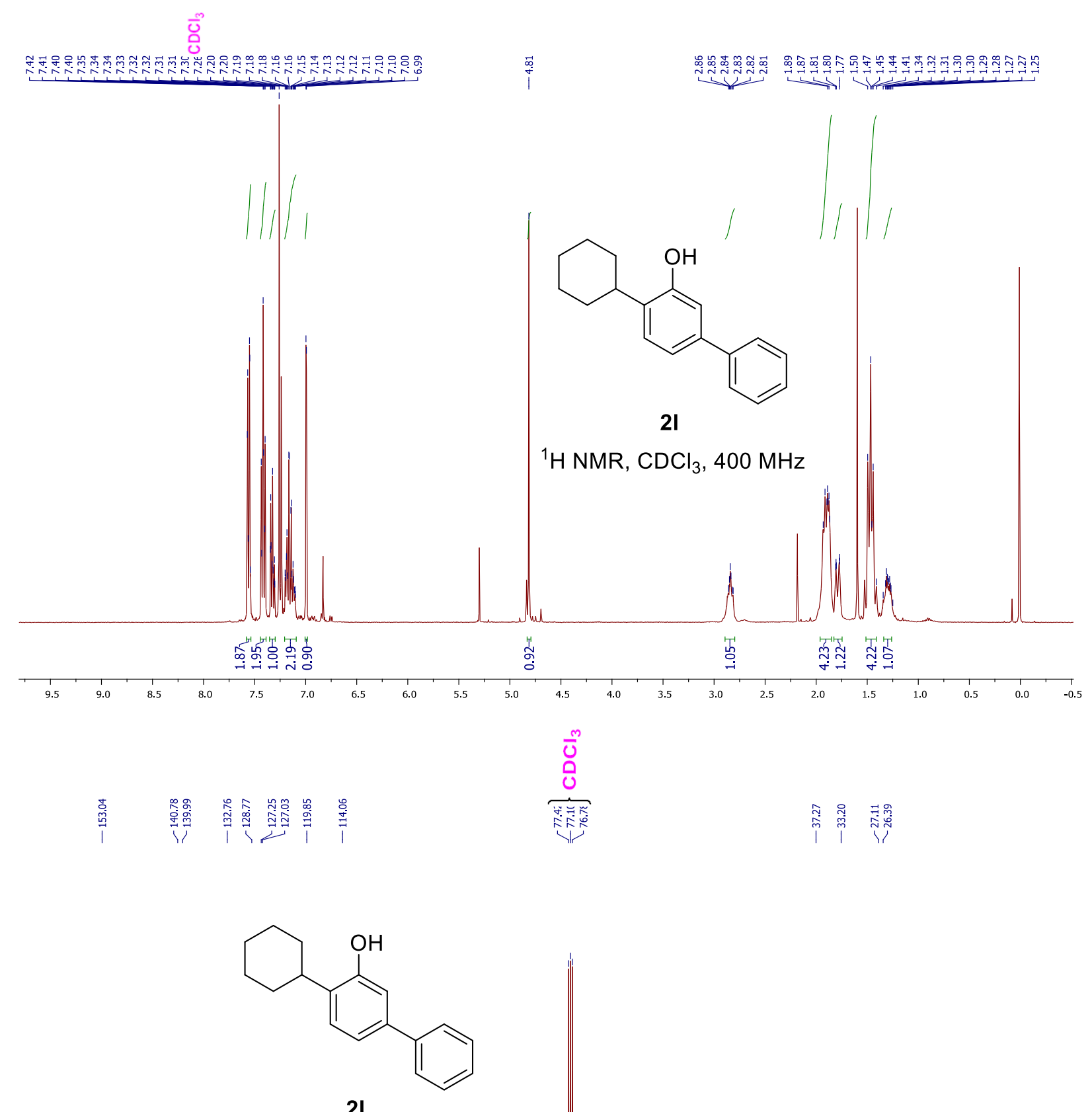

${ }^{13} \mathrm{C} \mathrm{NMR}, \mathrm{CDCl}_{3}, 101 \mathrm{MHz}$

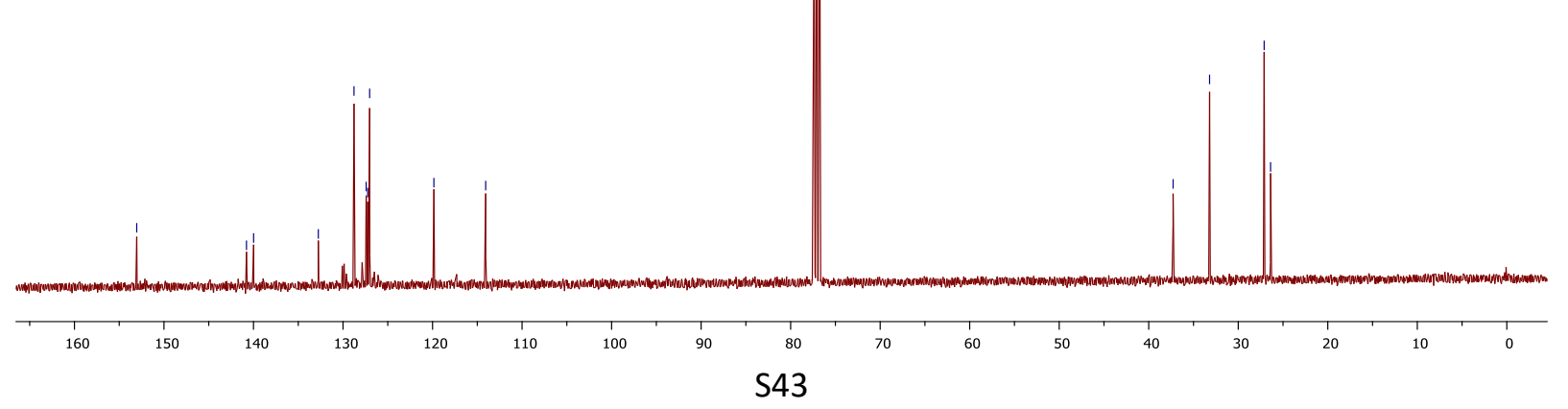




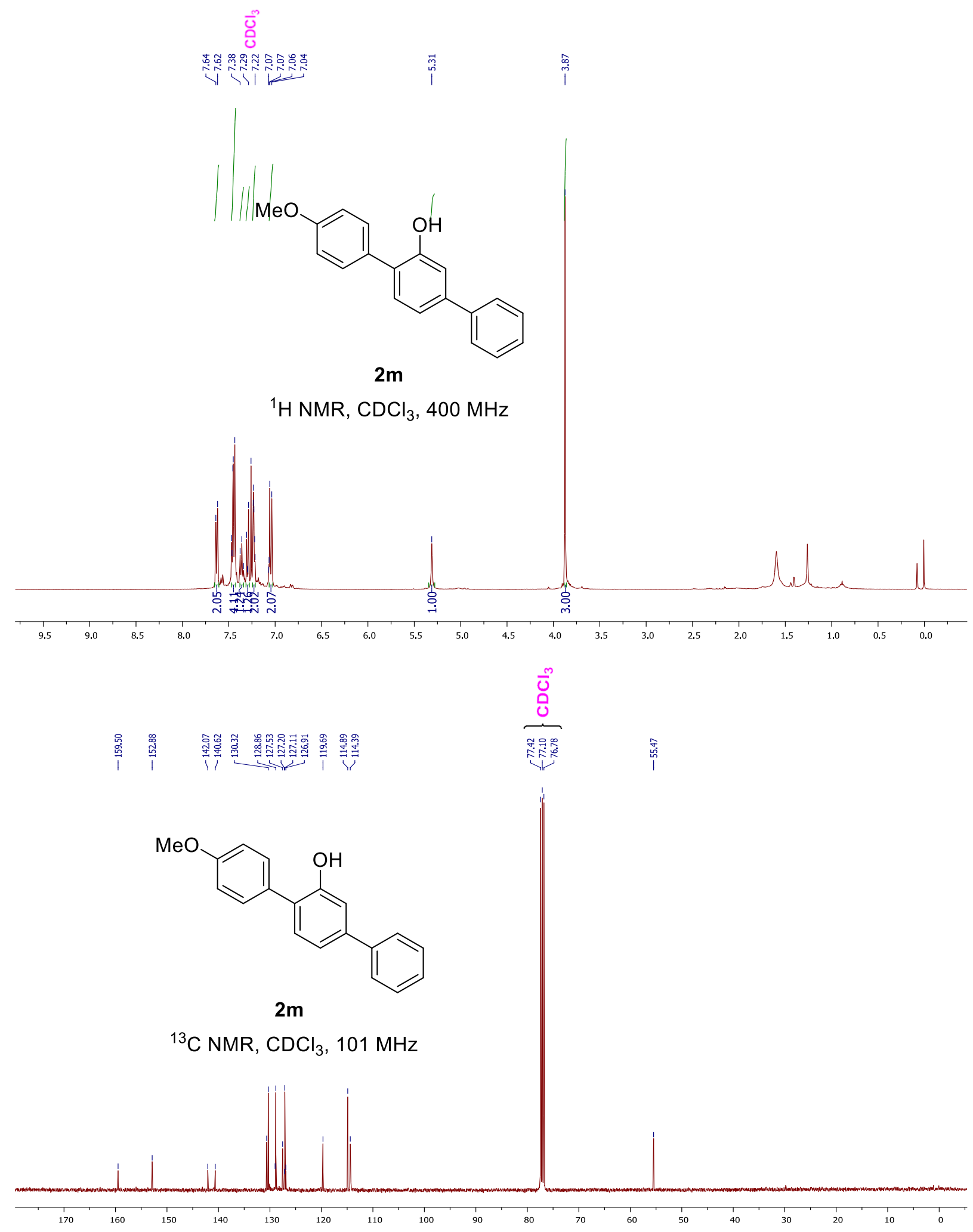


1111<smiles>CC(C)(C)c1cc(O)cc(-c2ccccc2)c1</smiles>

$2 n$

${ }^{1} \mathrm{H}$ NMR, $\mathrm{CDCl}_{3}, 400 \mathrm{MHz}$
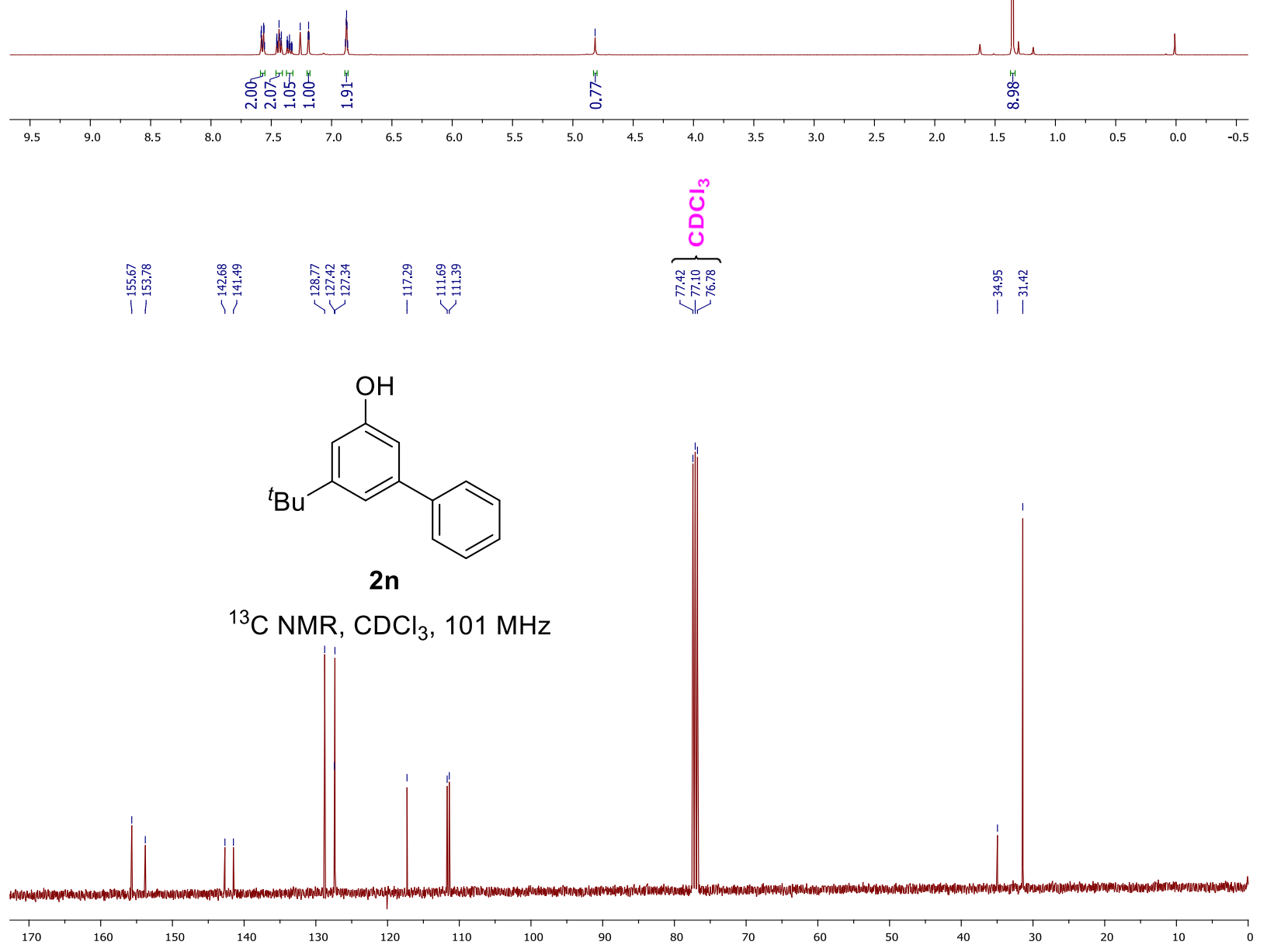

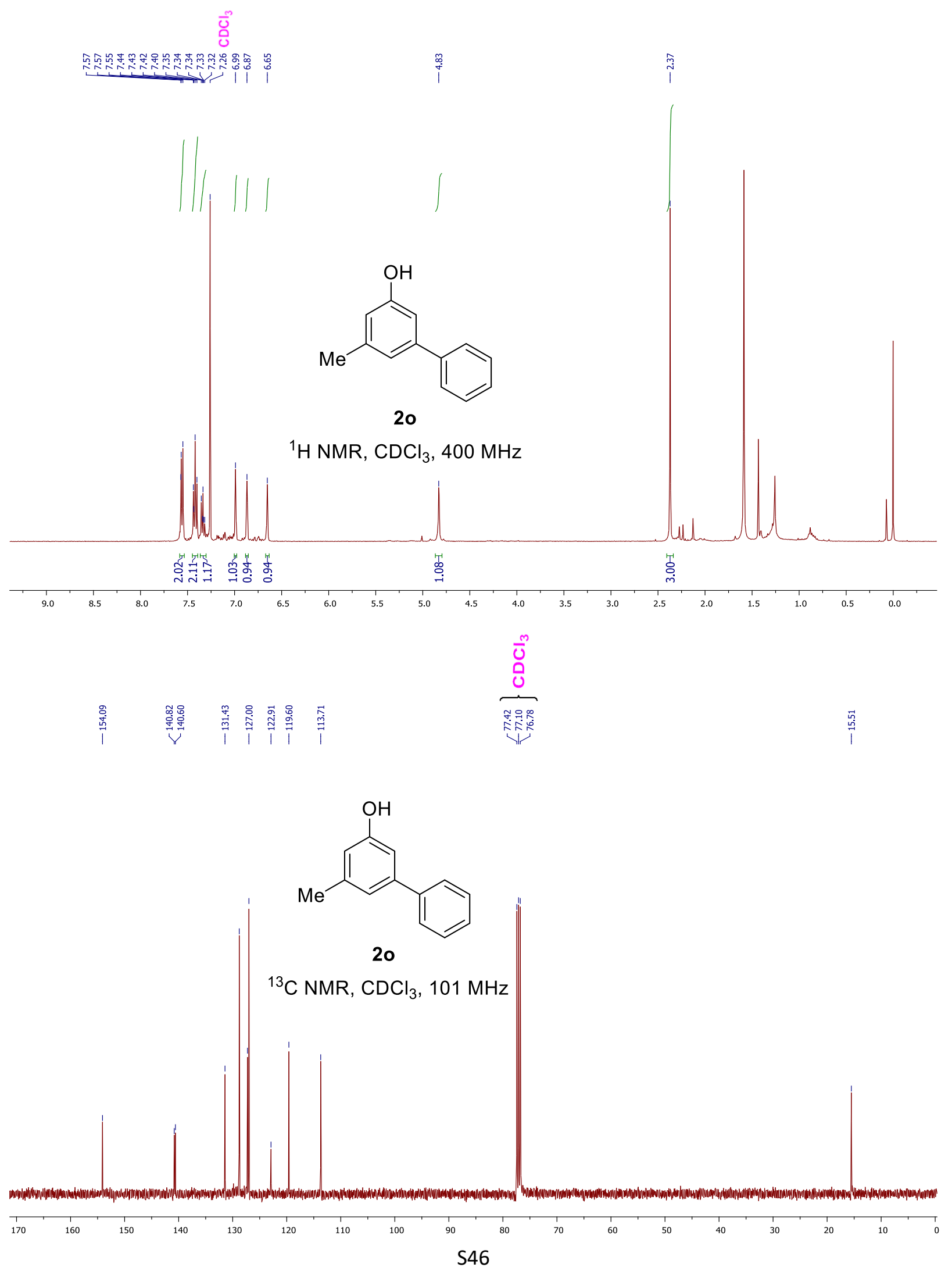


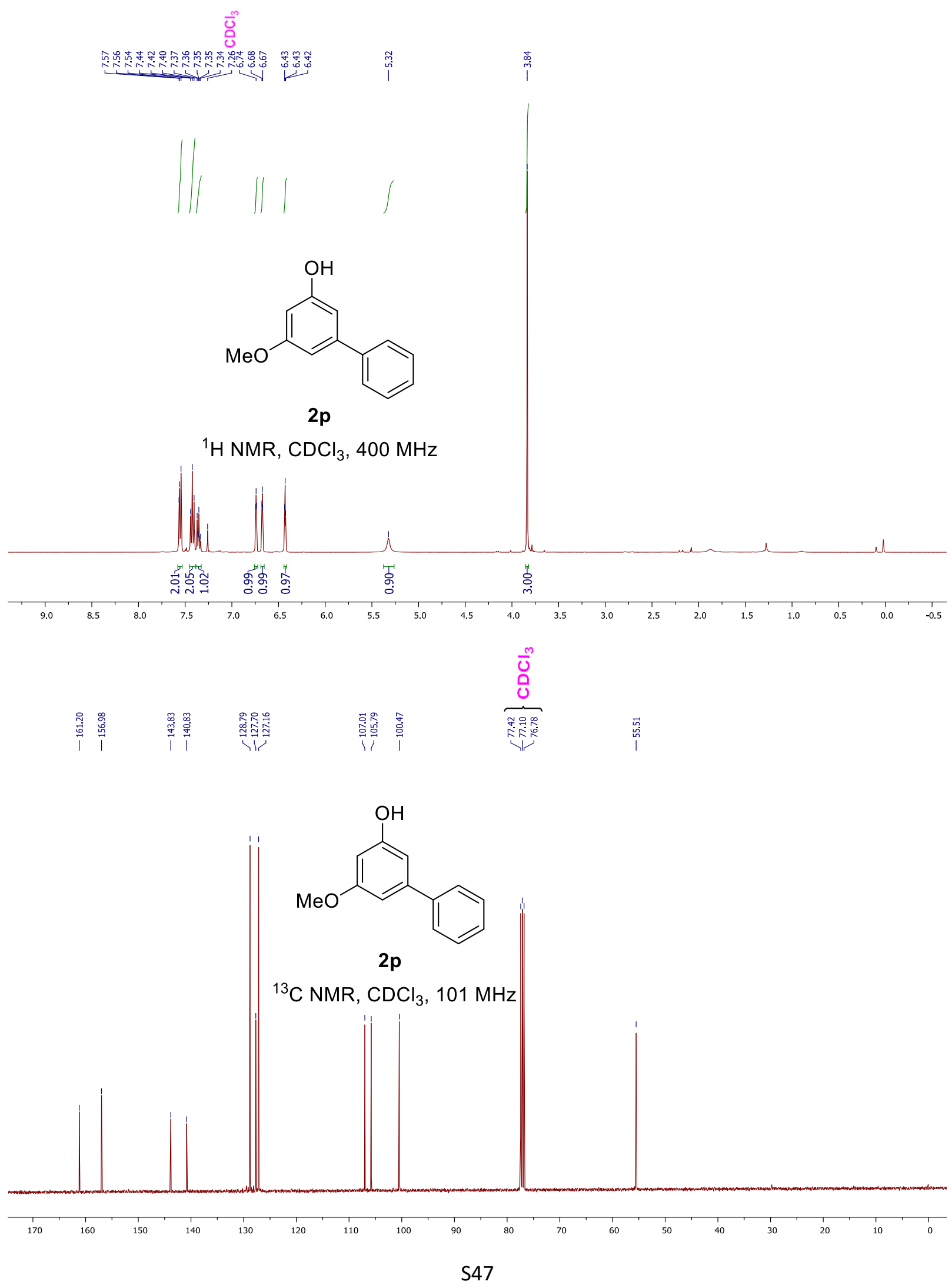




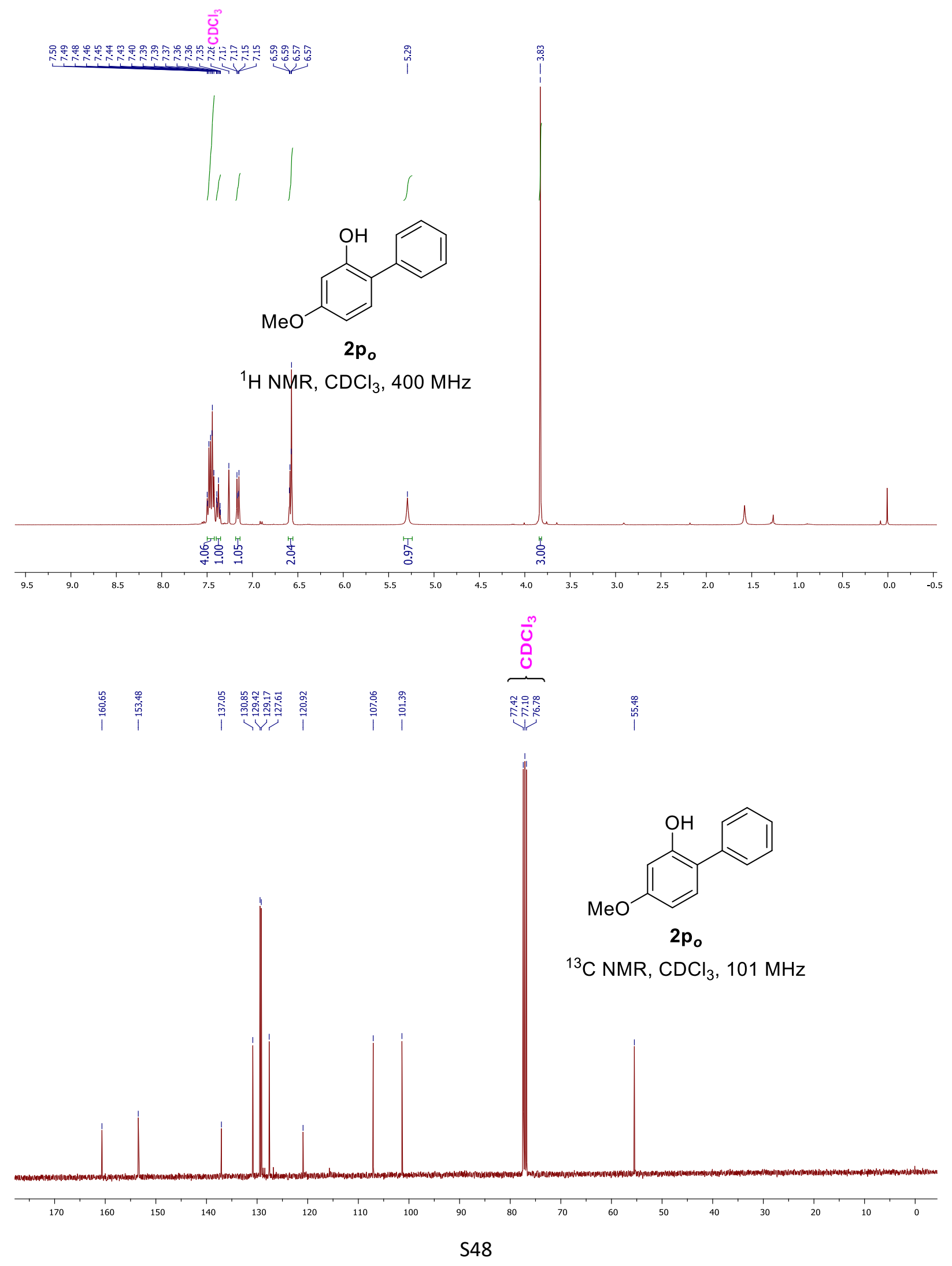




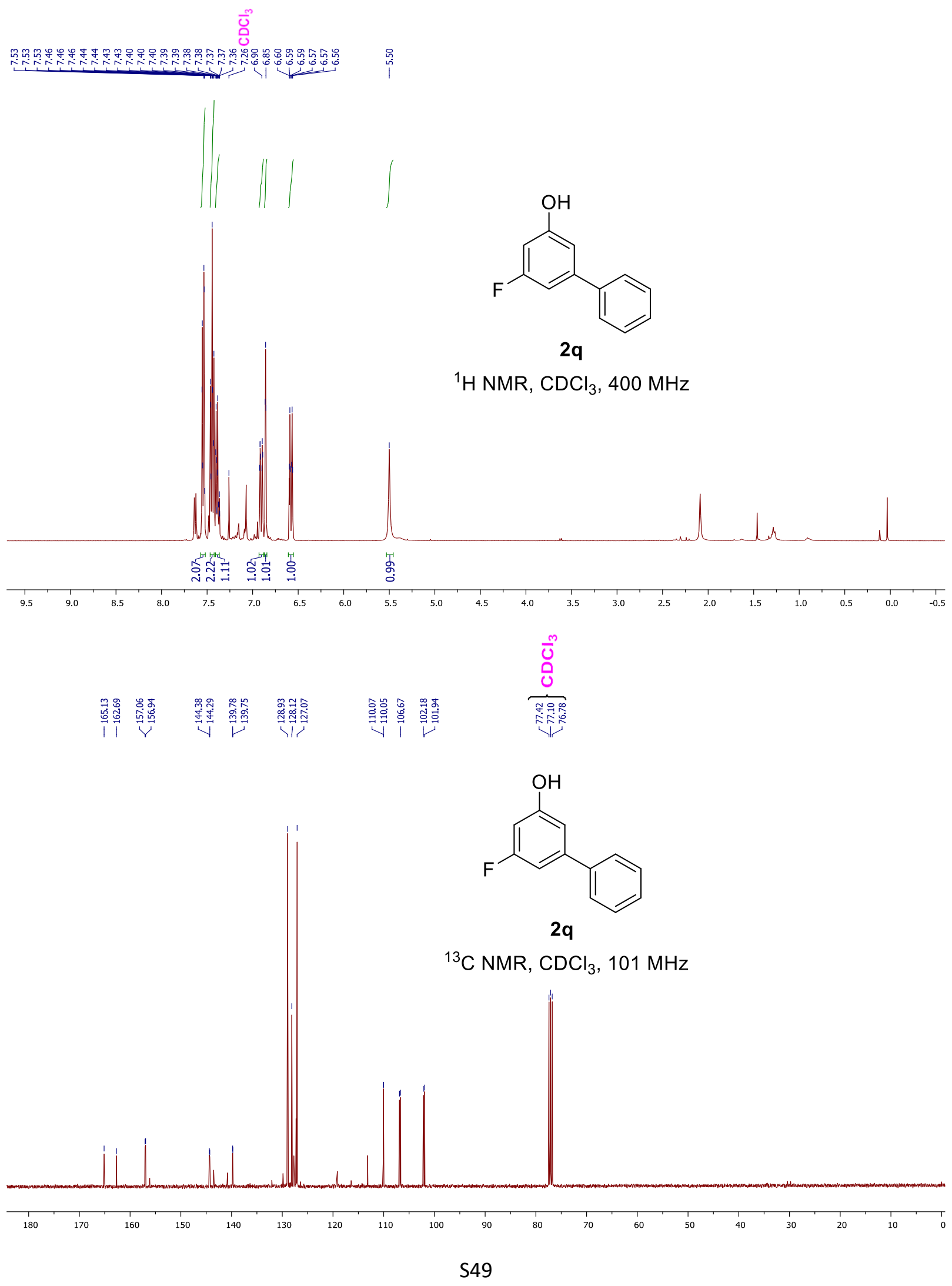




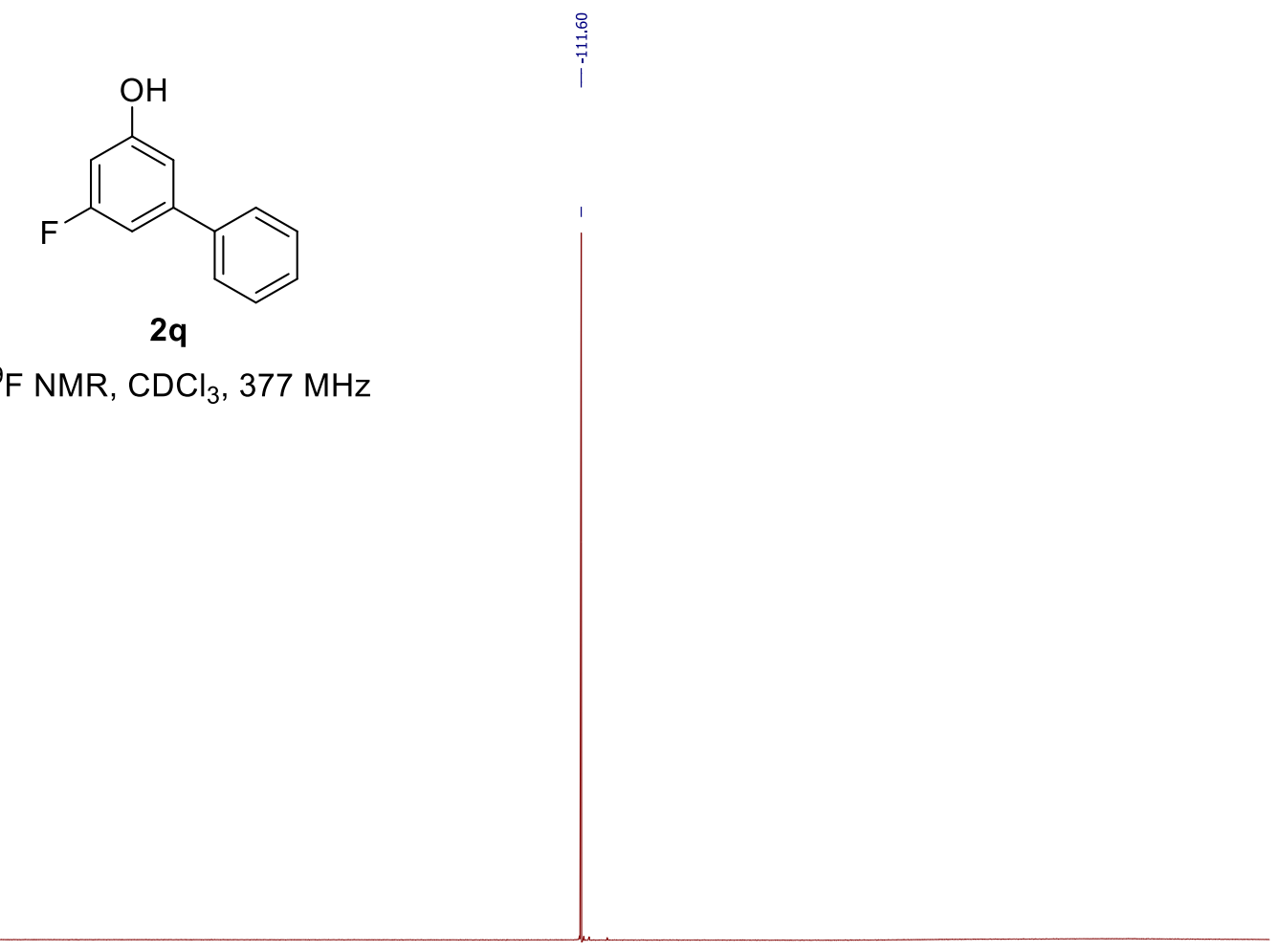

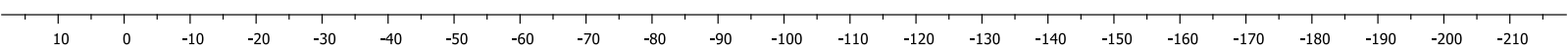



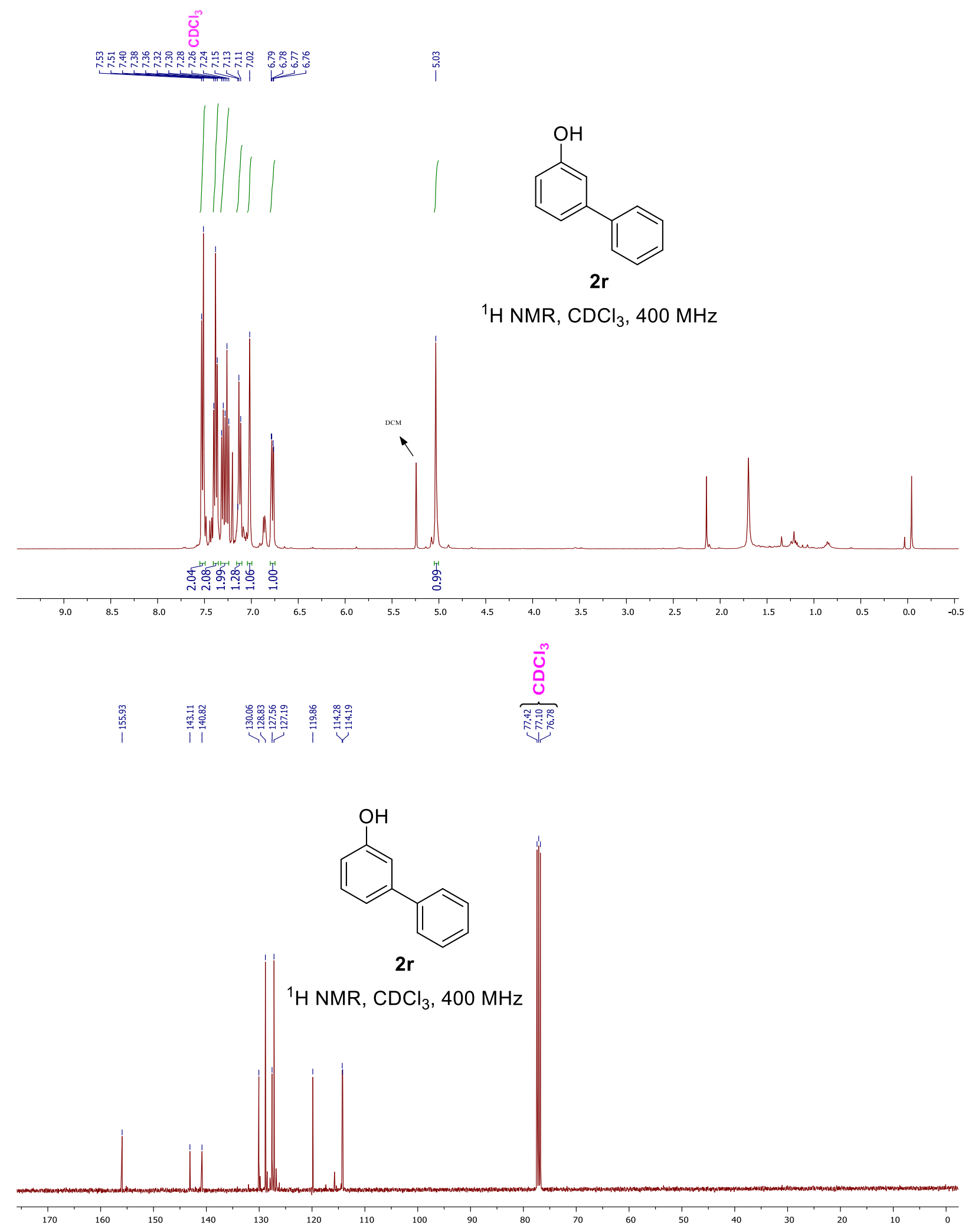


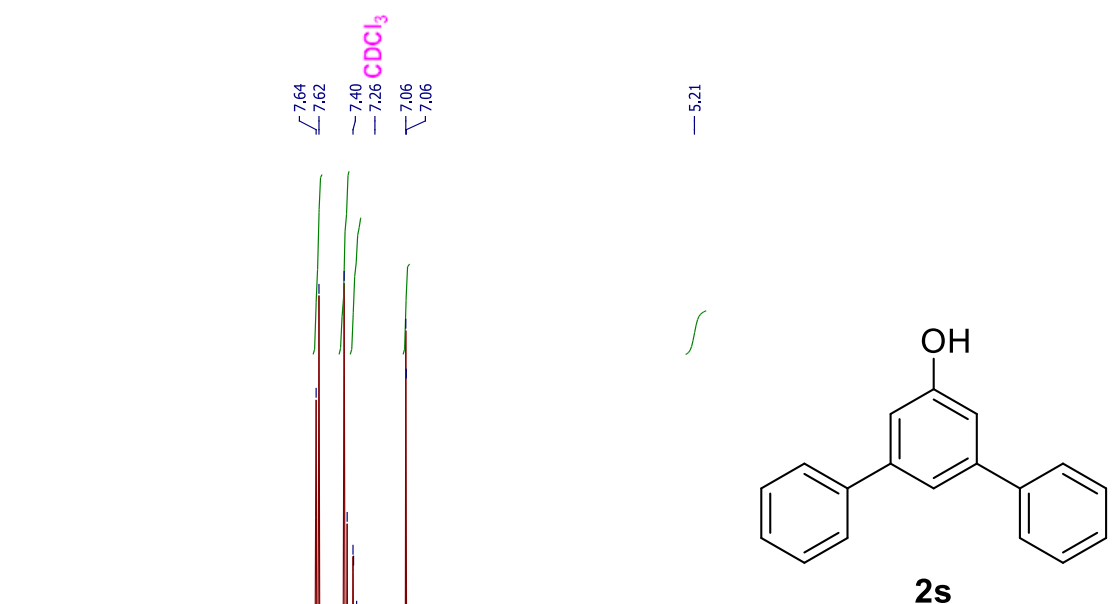

${ }^{1} \mathrm{H} \mathrm{NMR}, \mathrm{CDCl}_{3}, 400 \mathrm{MHz}$

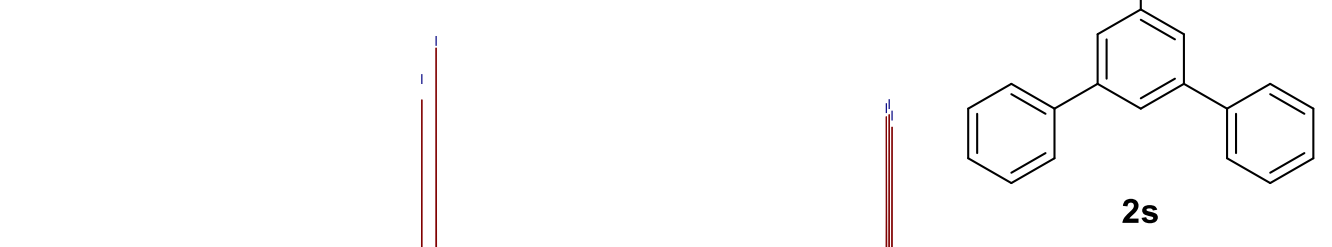

${ }^{13} \mathrm{C} \mathrm{NMR}, \mathrm{CDCl}_{3}, 101 \mathrm{MHz}$ 

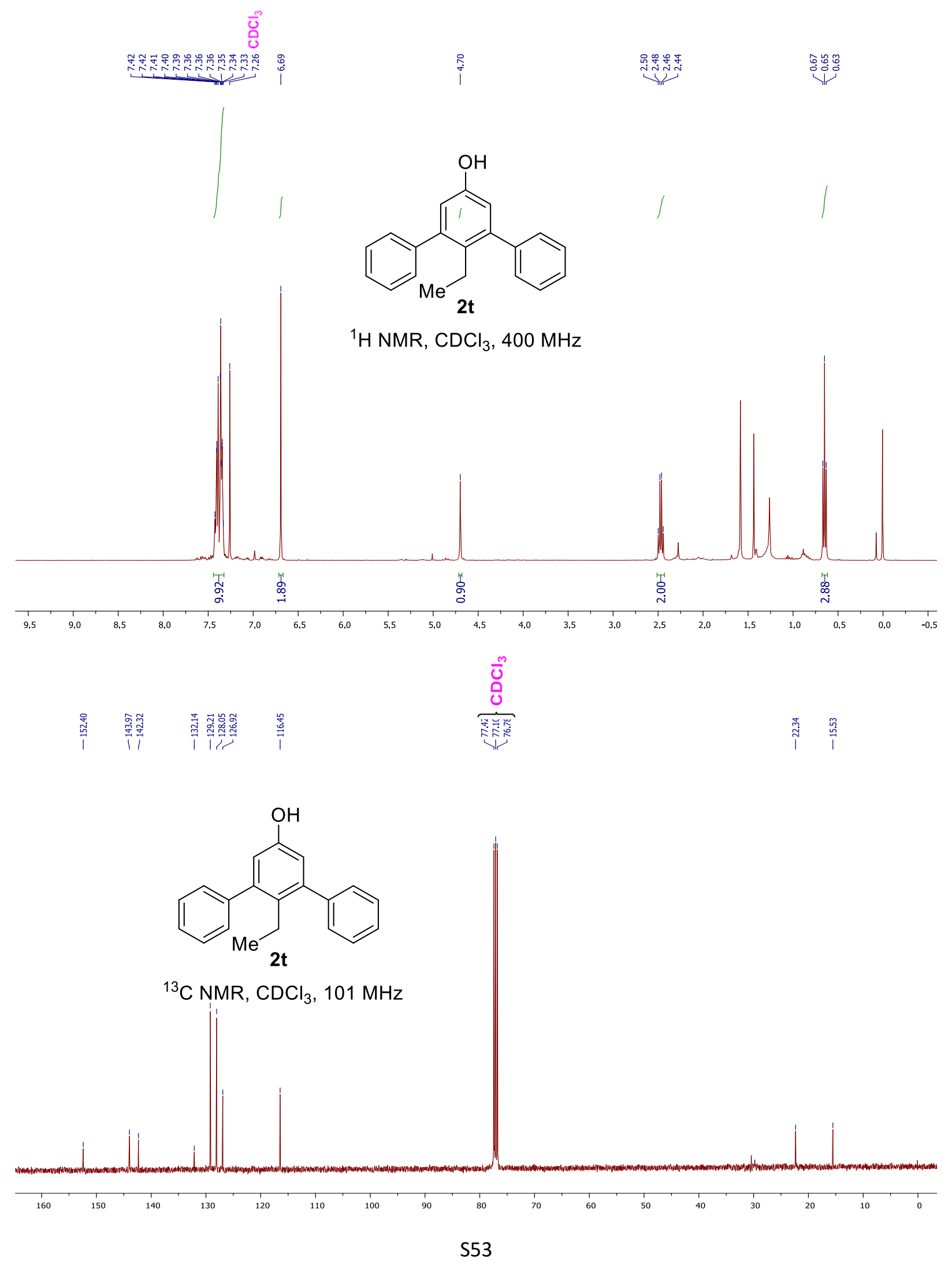


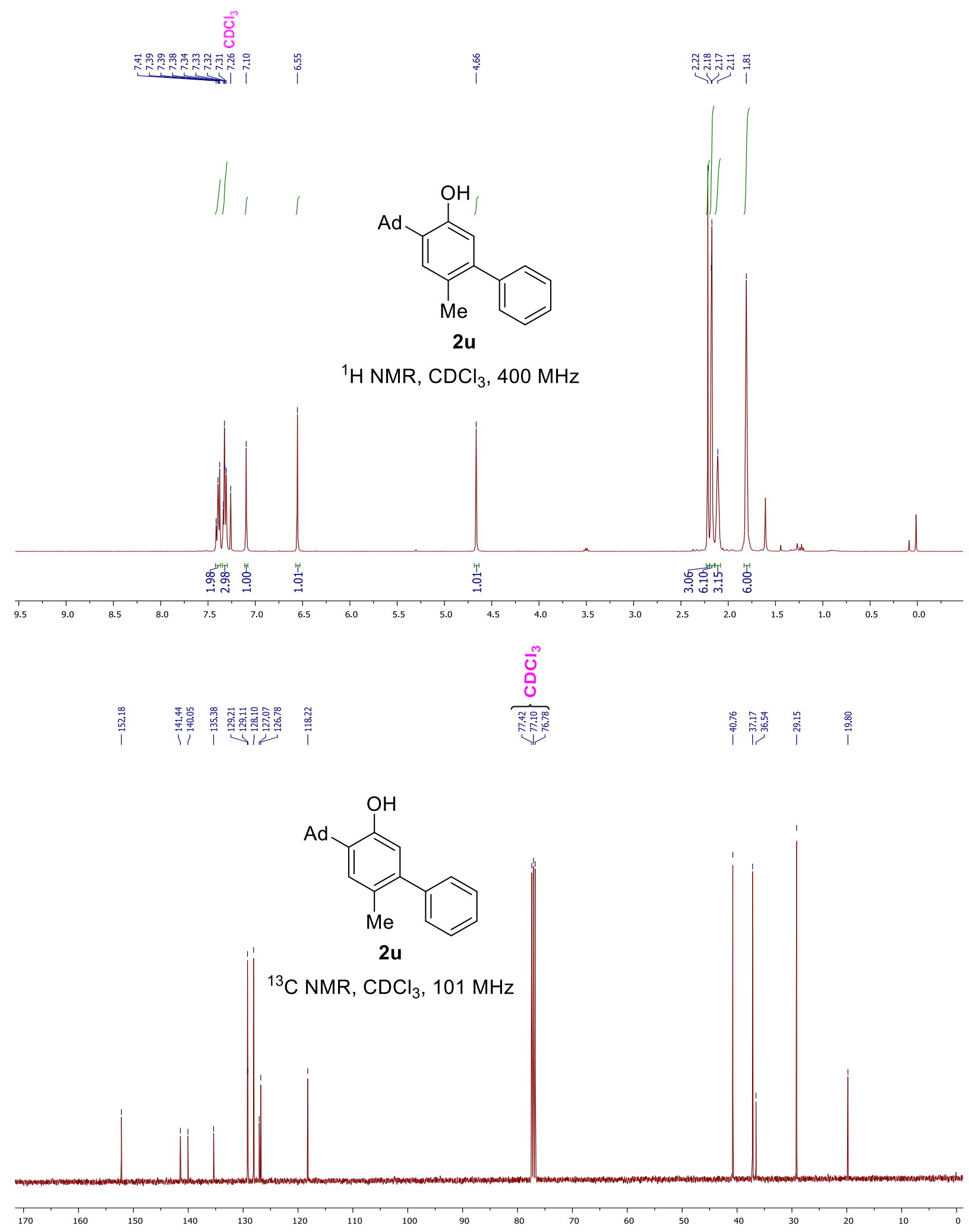




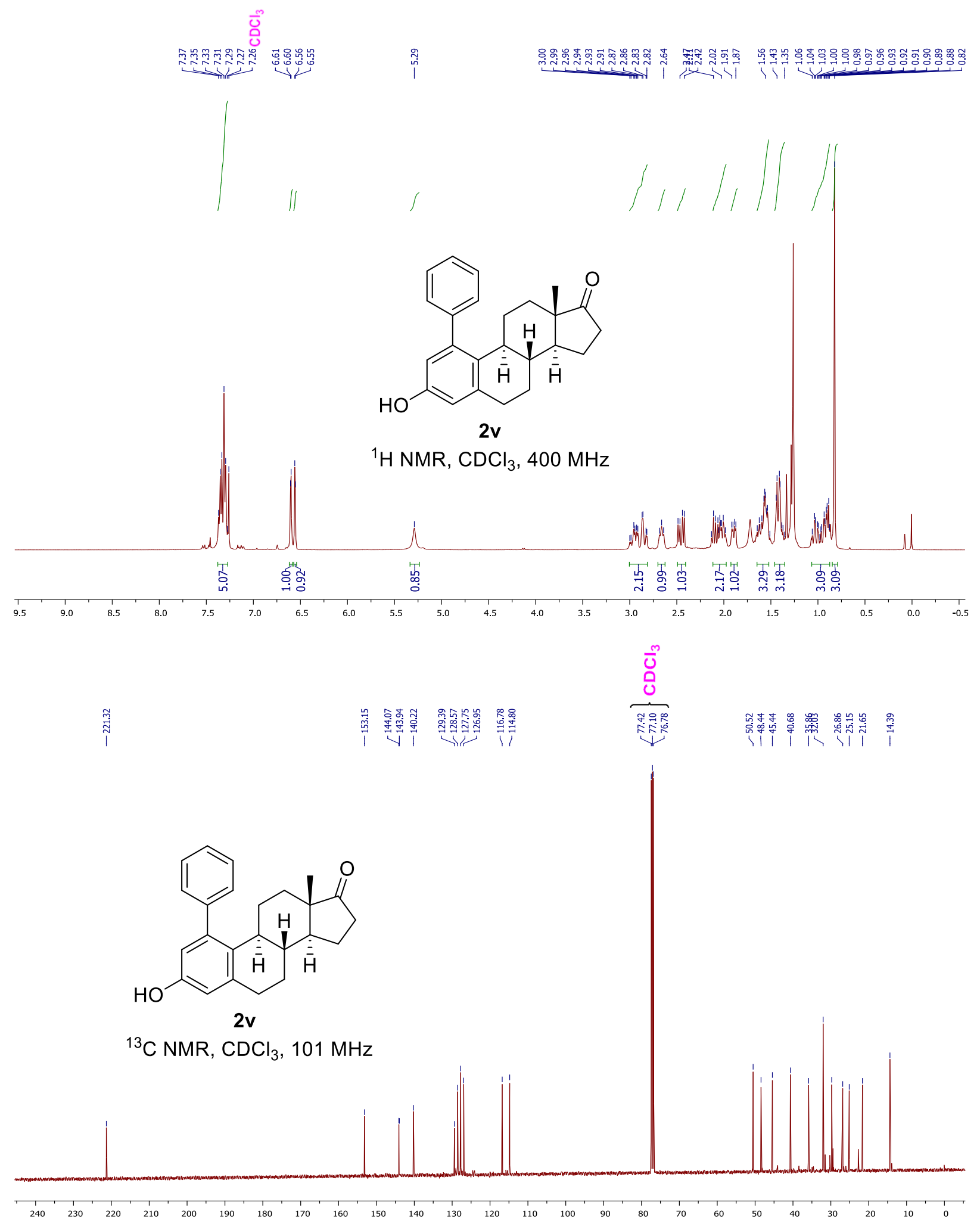



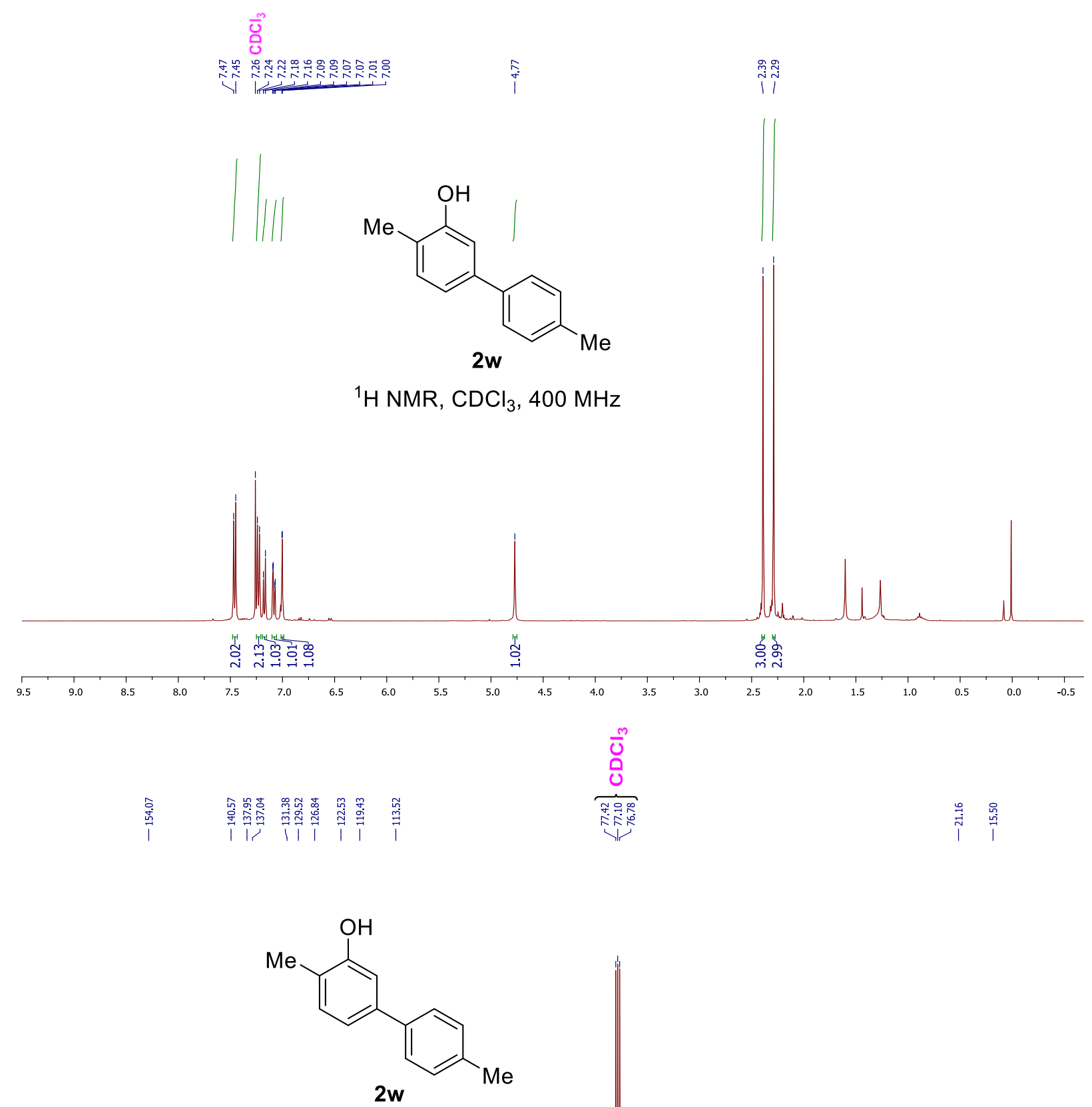

${ }^{13} \mathrm{C} \mathrm{NMR}, \mathrm{CDCl}_{3}, 101 \mathrm{MHz}$

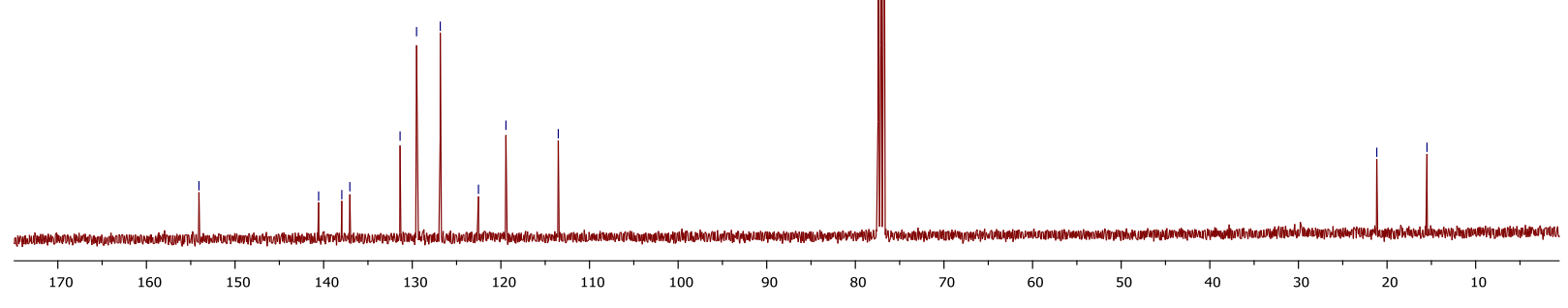




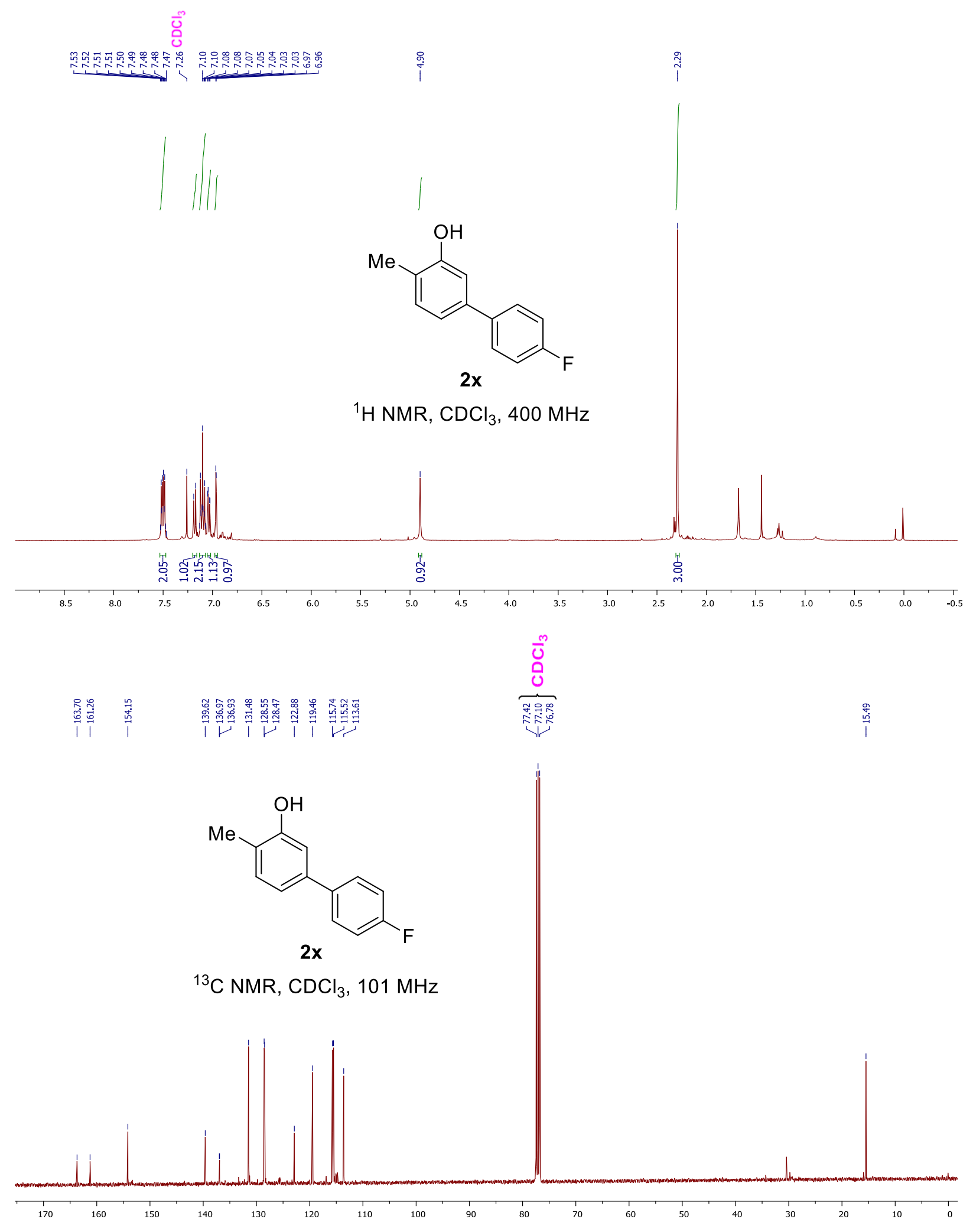




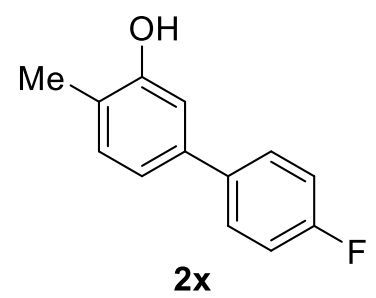

${ }^{19} \mathrm{~F} \mathrm{NMR}, \mathrm{CDCl}_{3}, 377 \mathrm{MHz}$

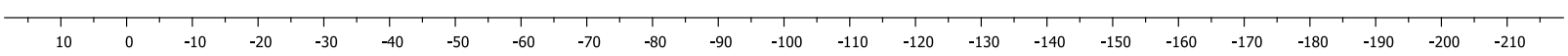




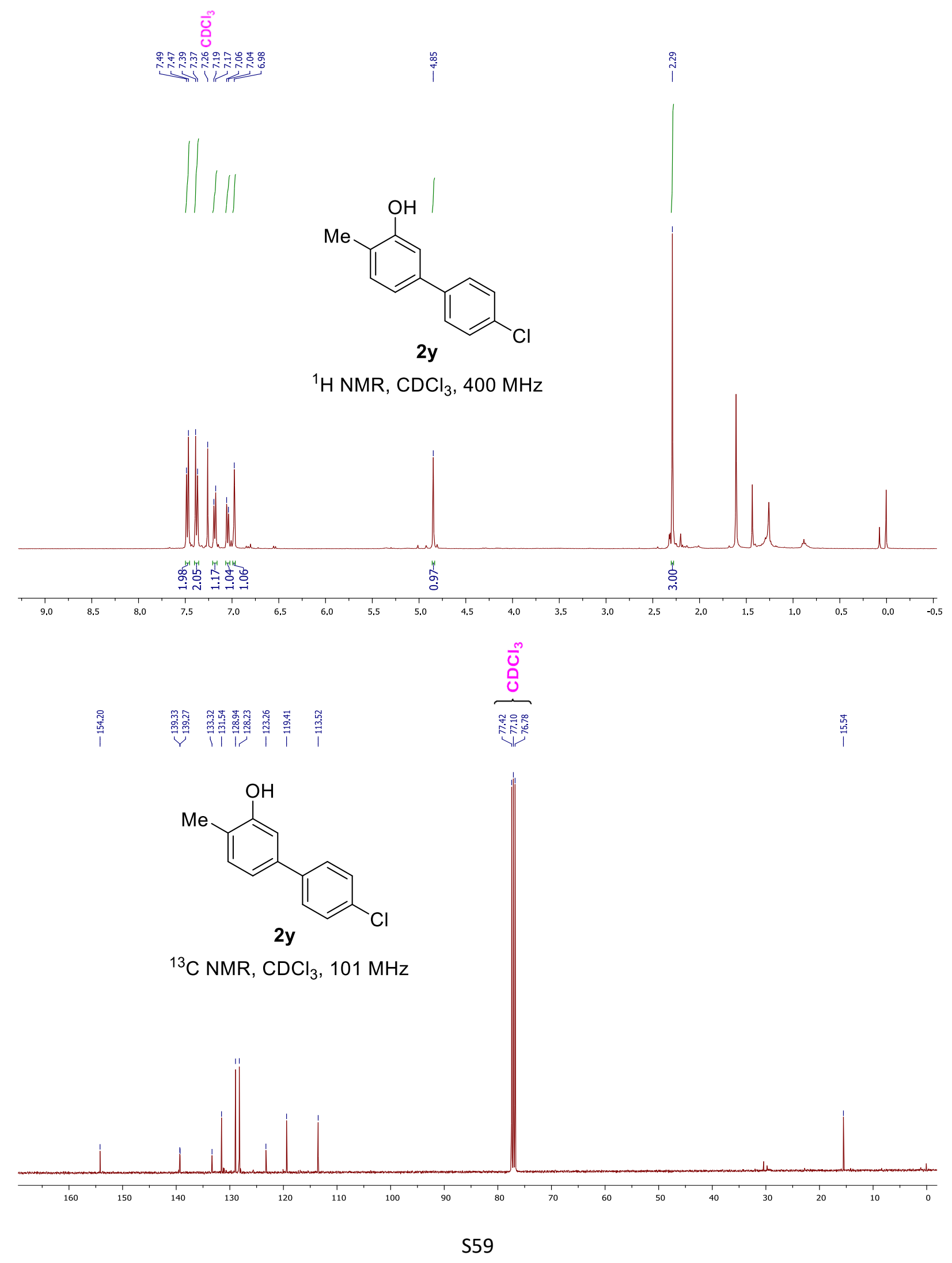




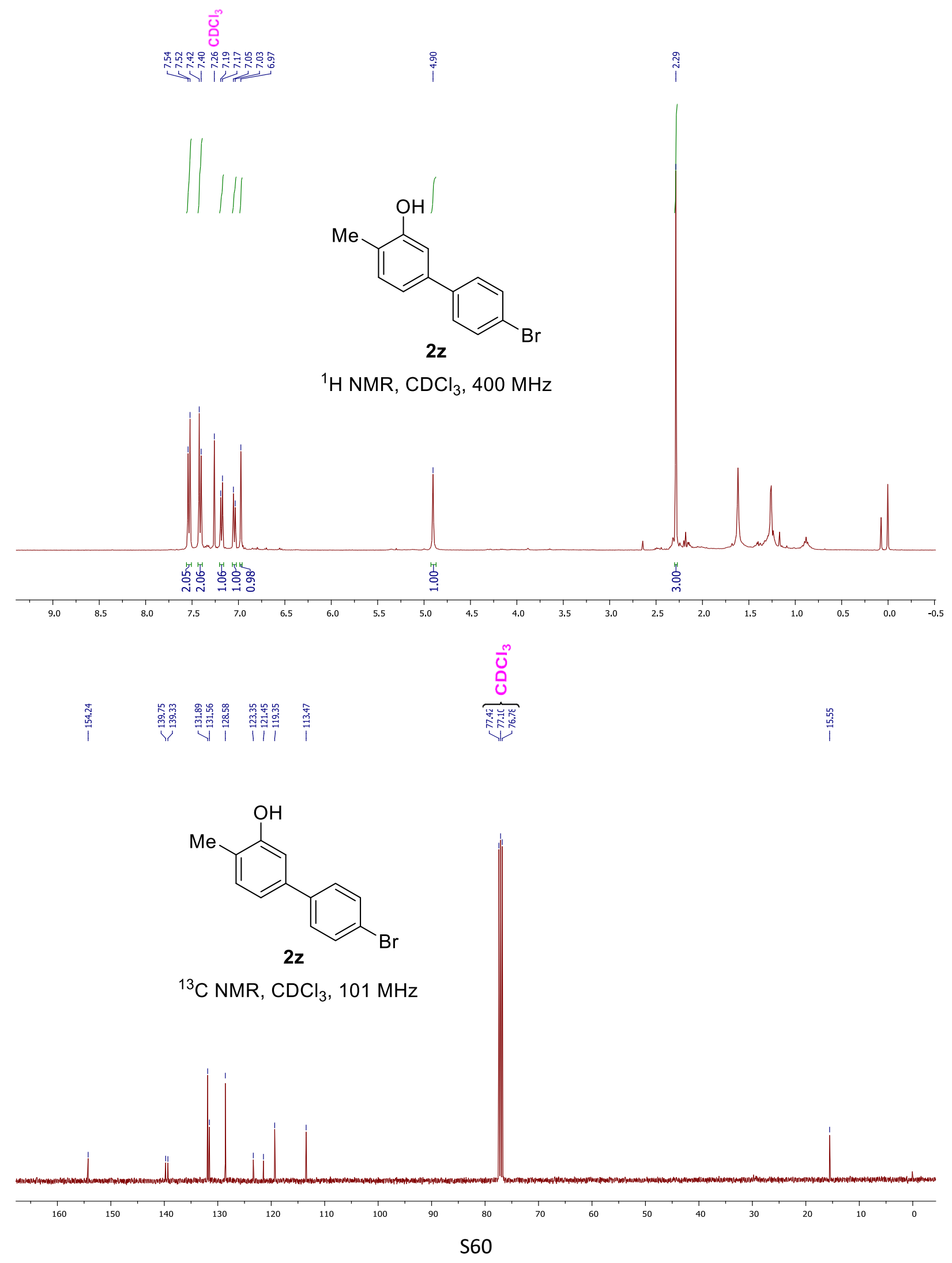




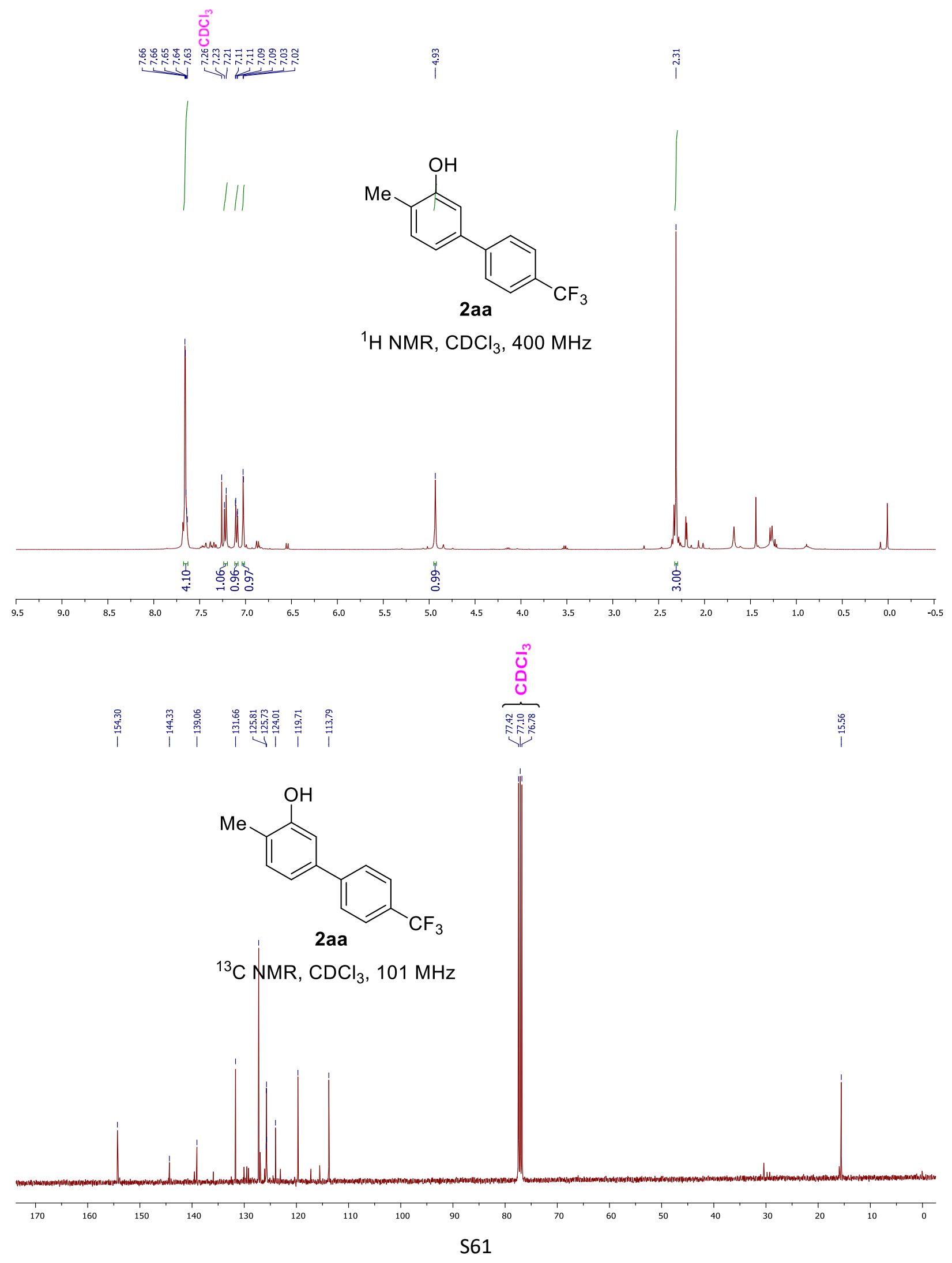


్ํำ
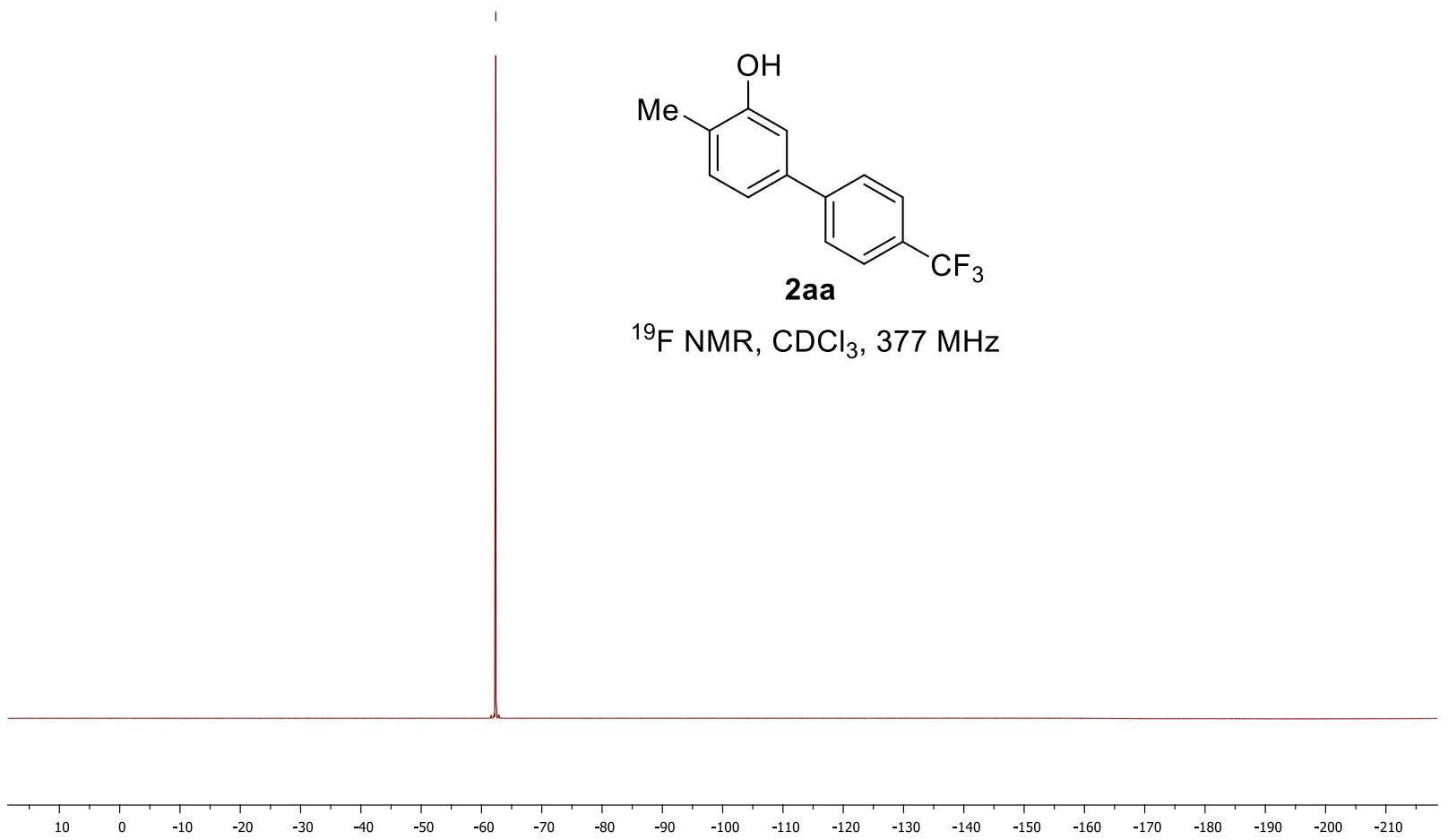

S62 


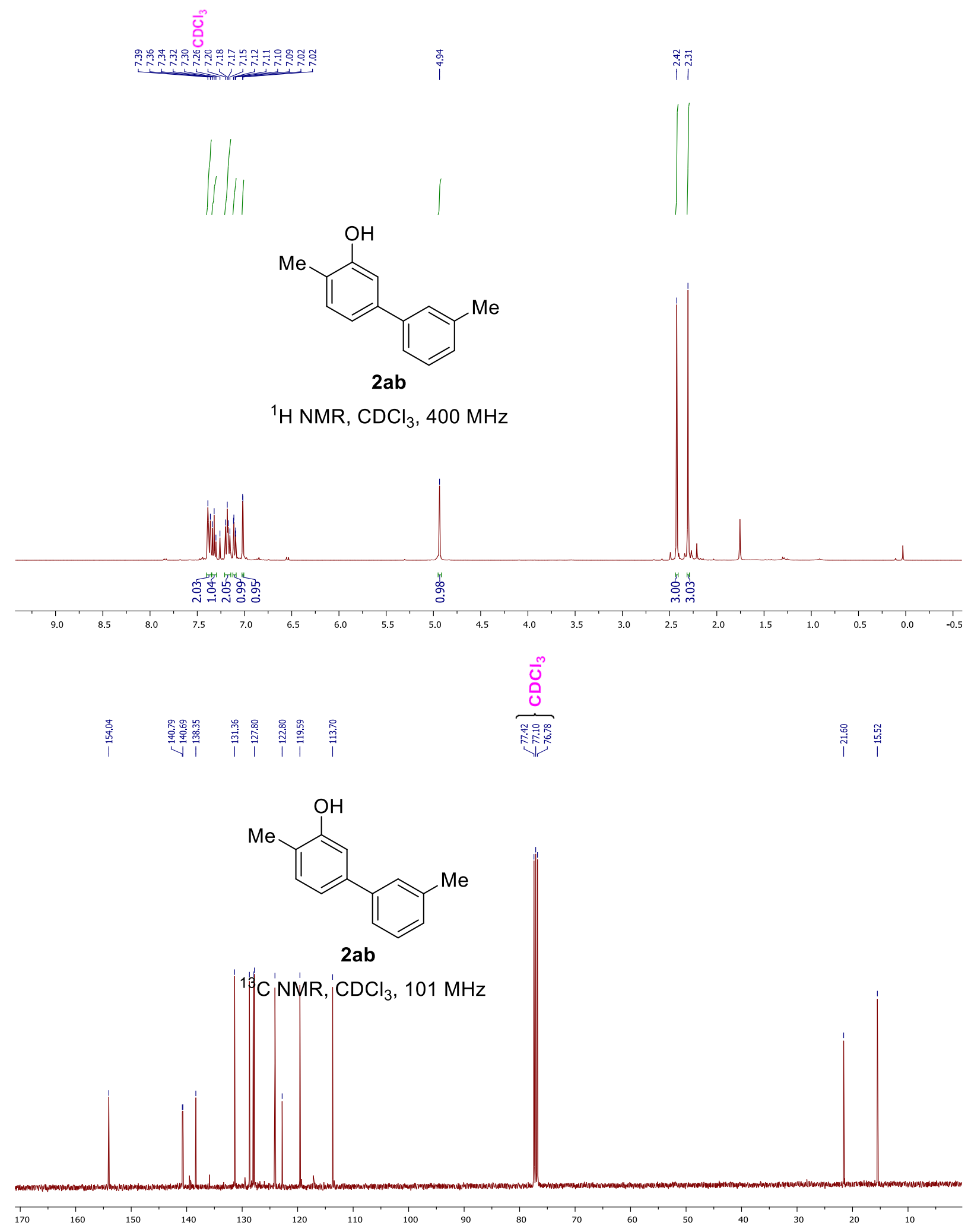




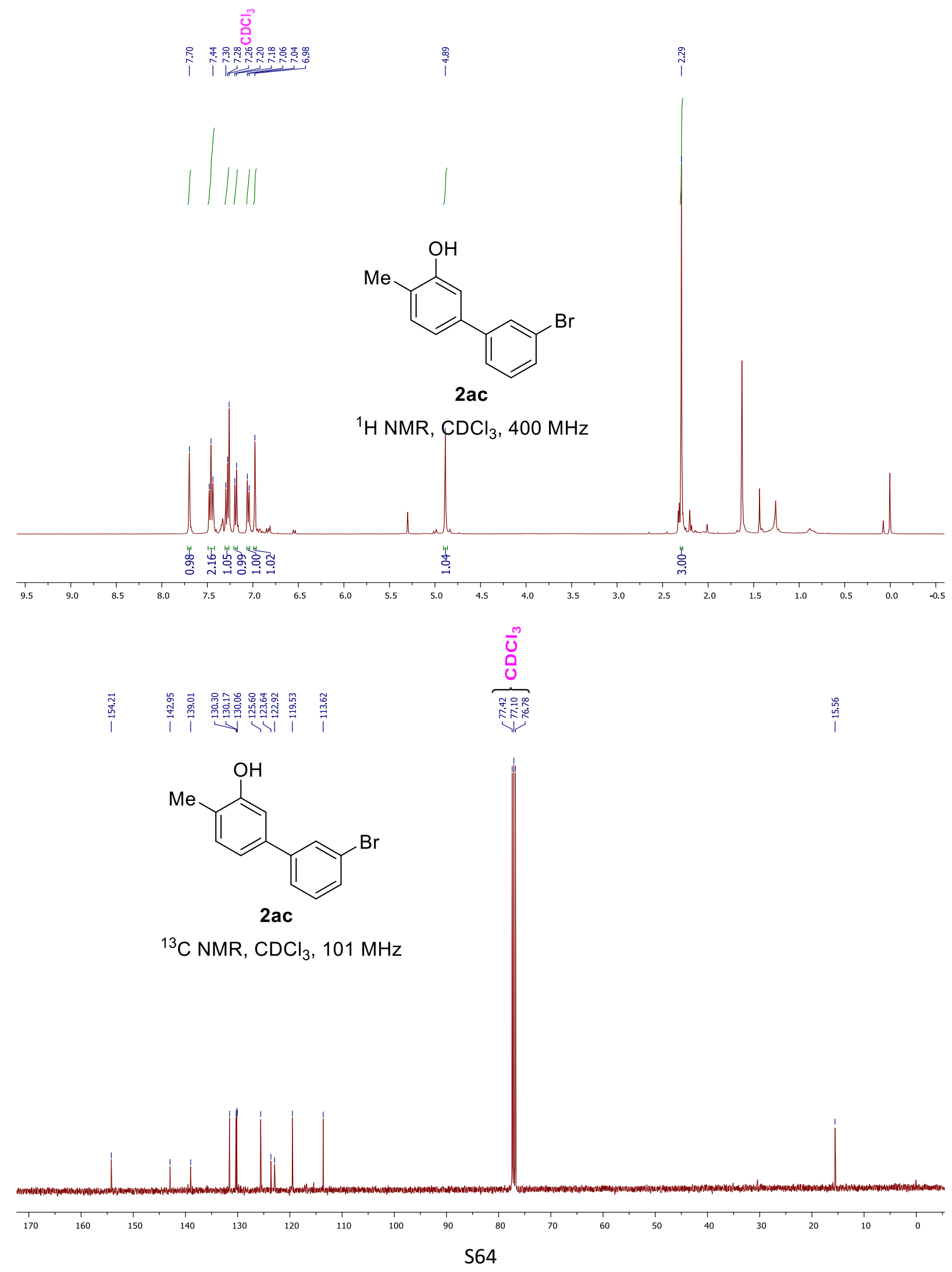



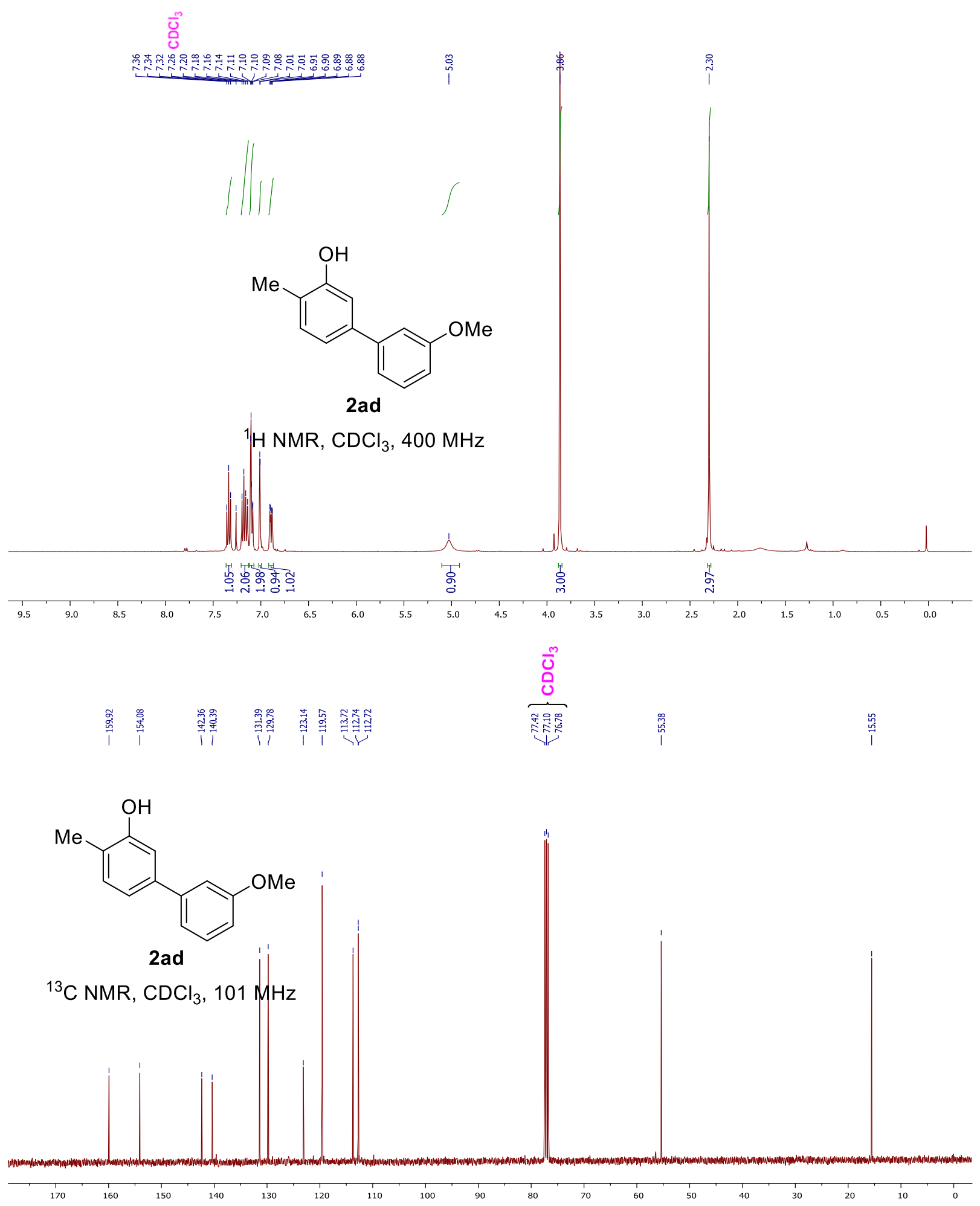


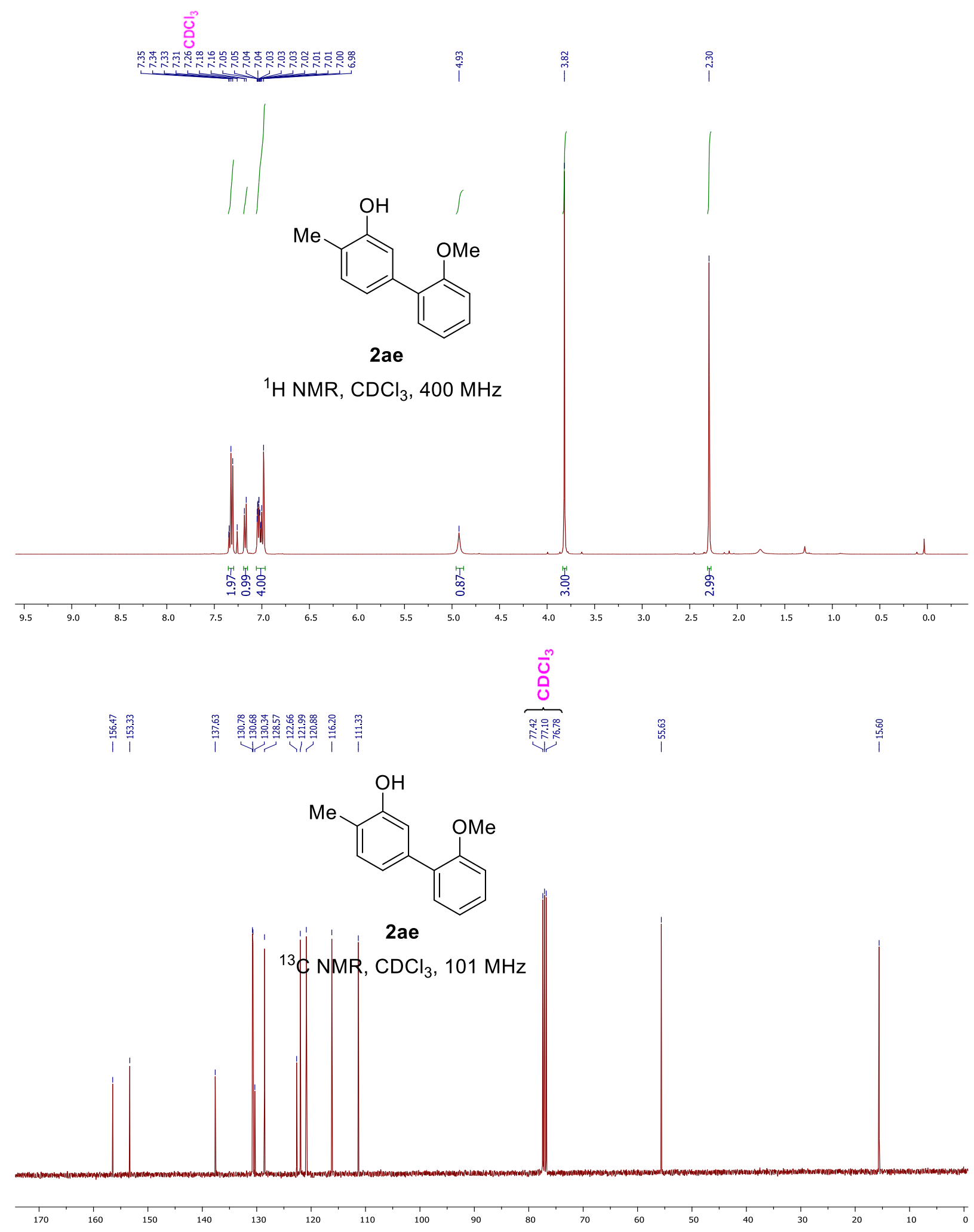




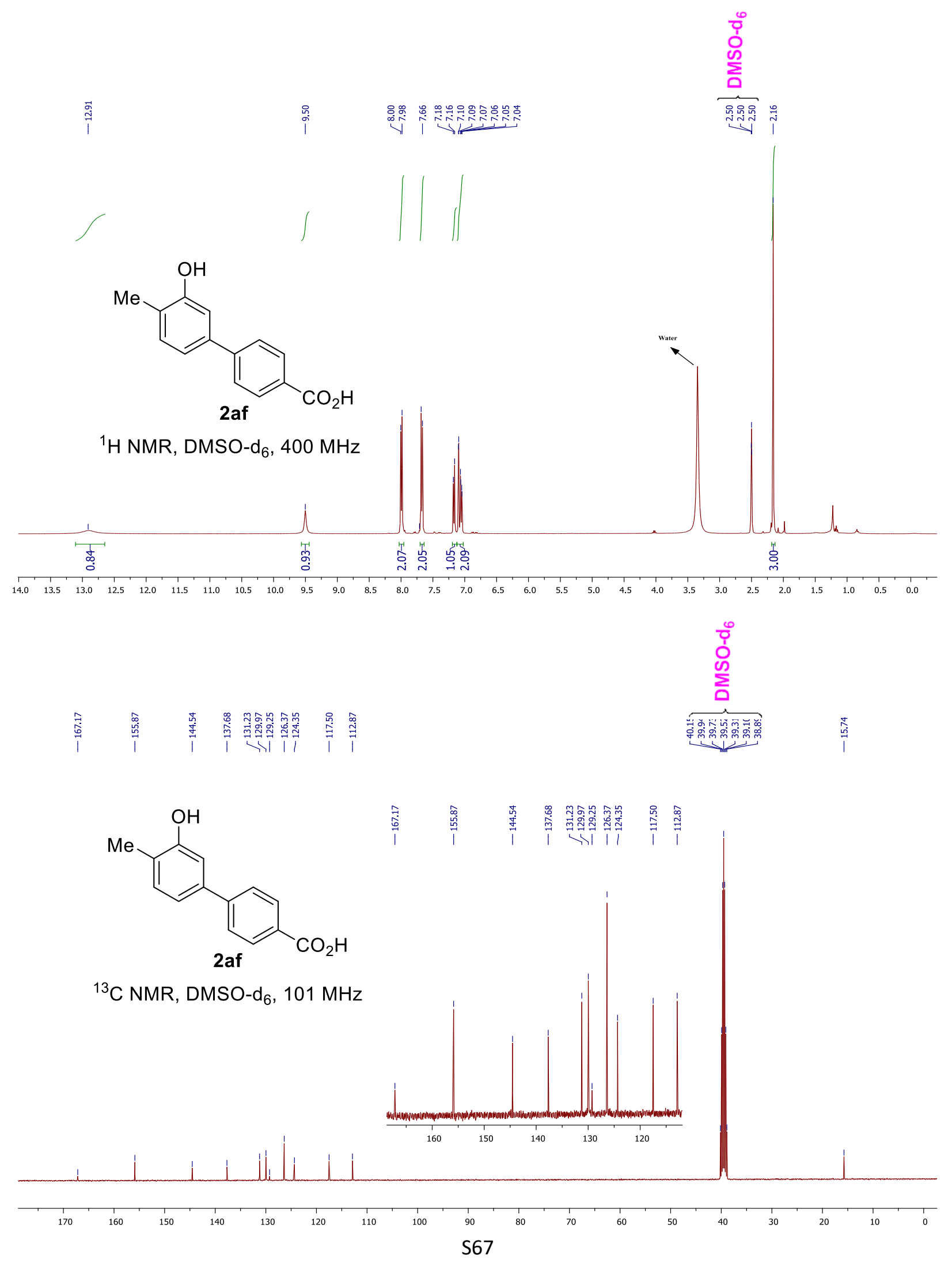




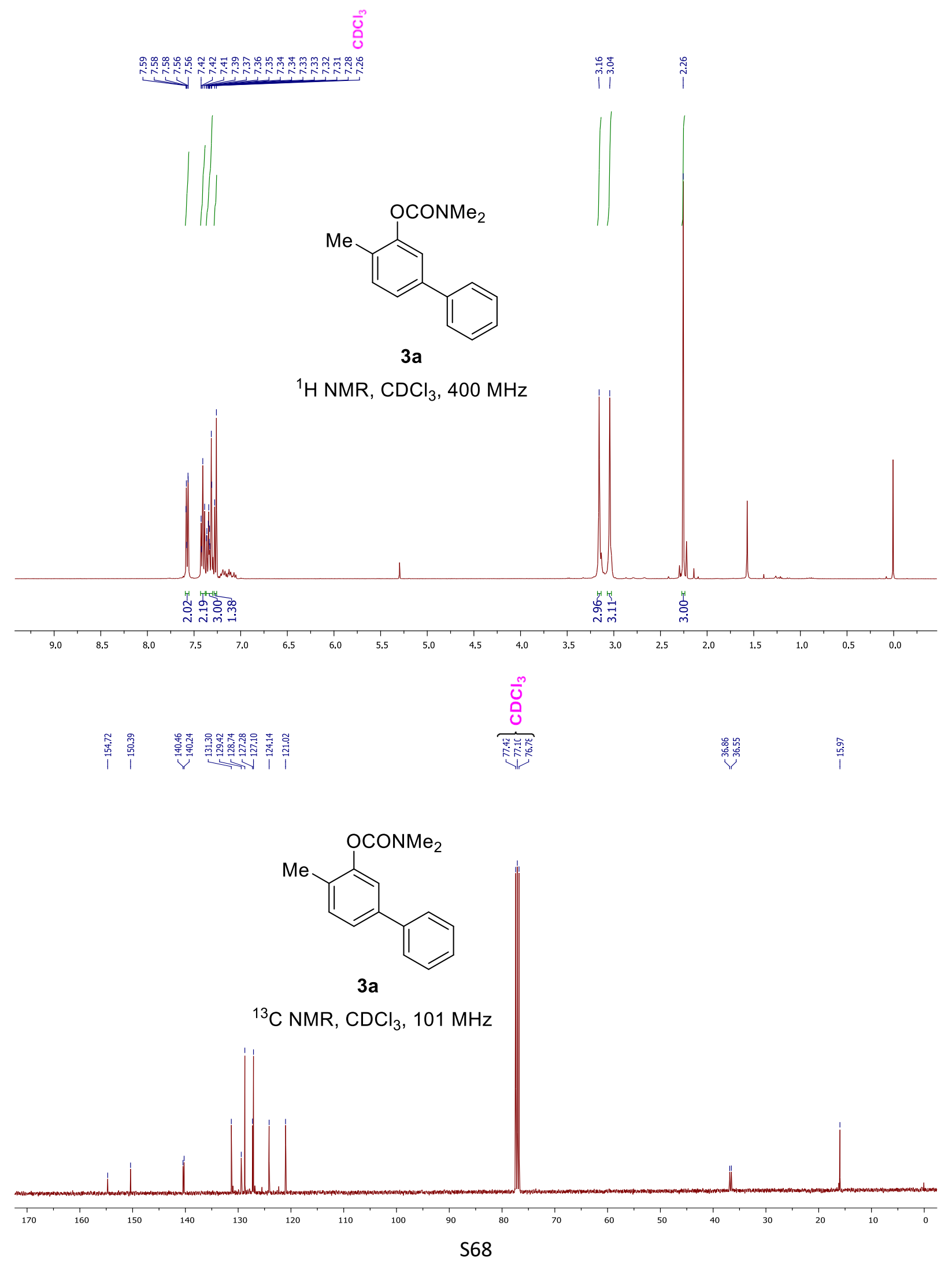




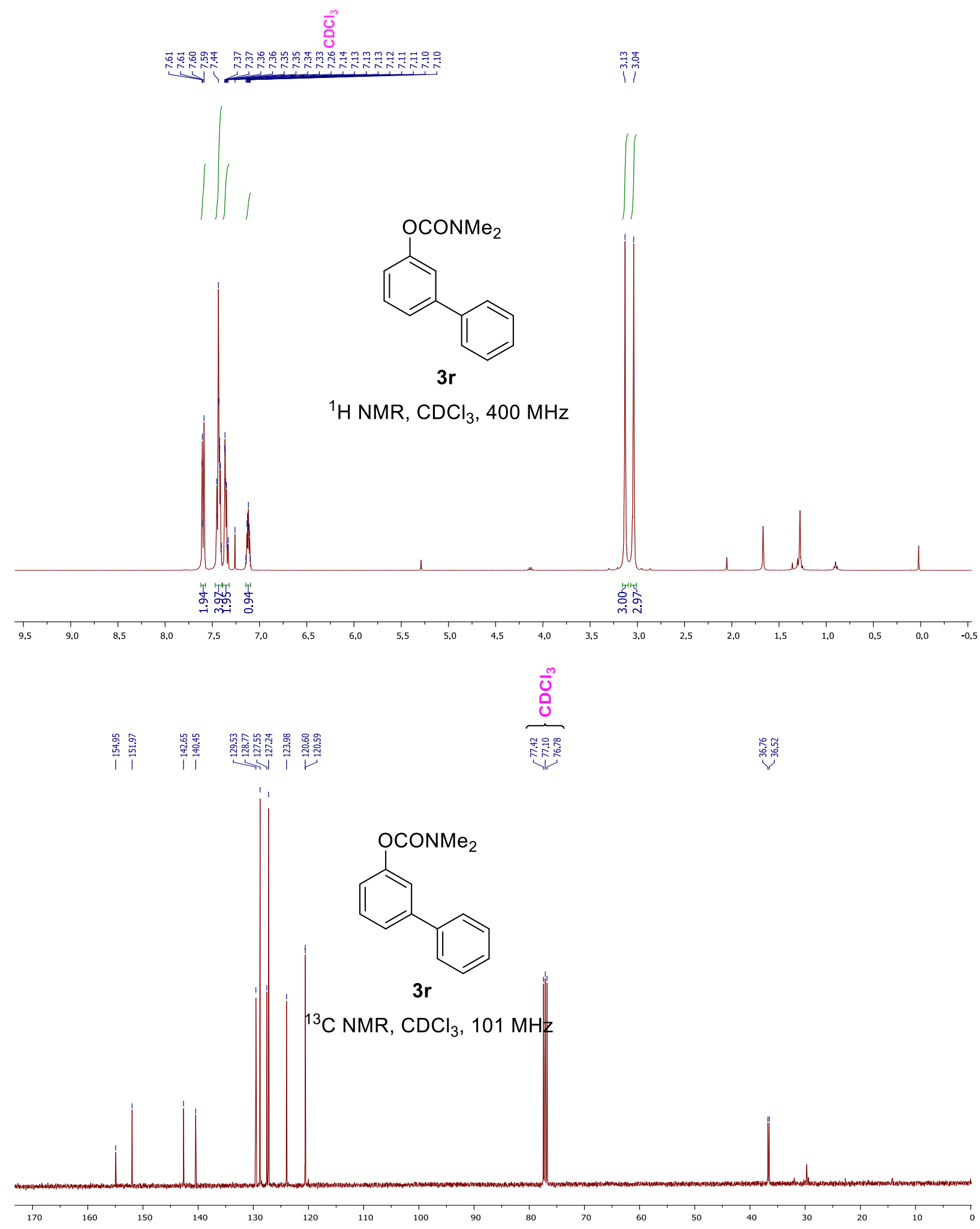




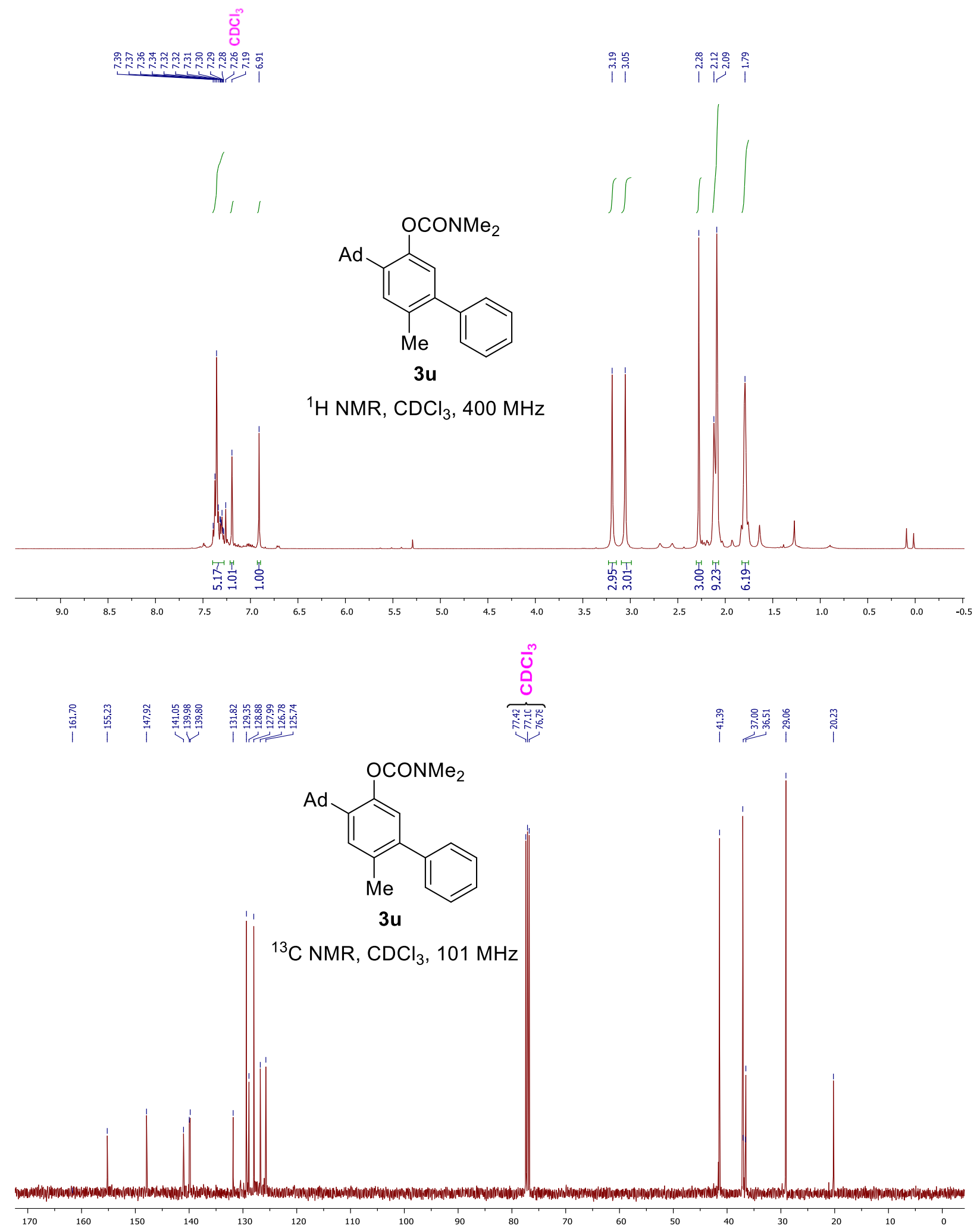




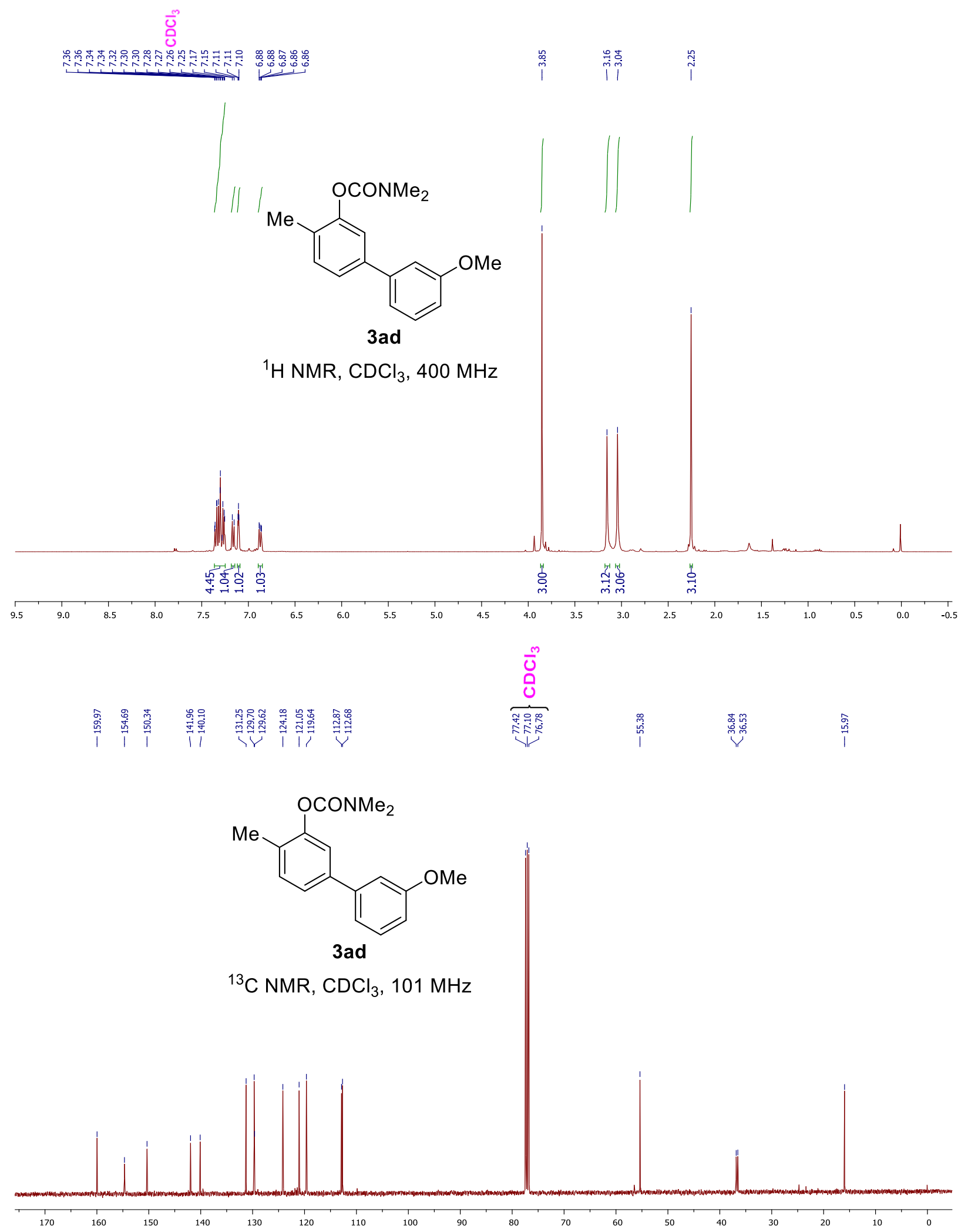




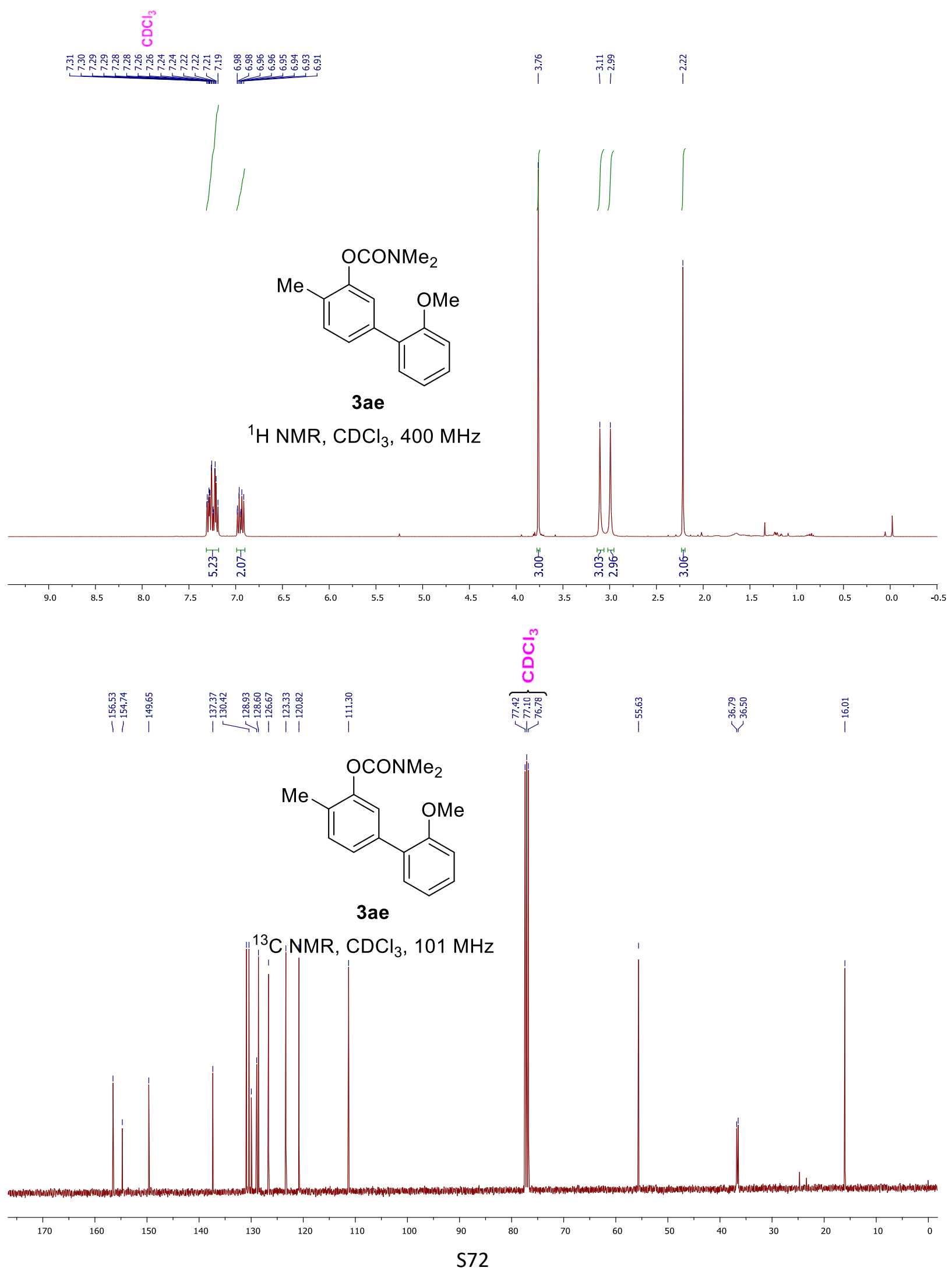



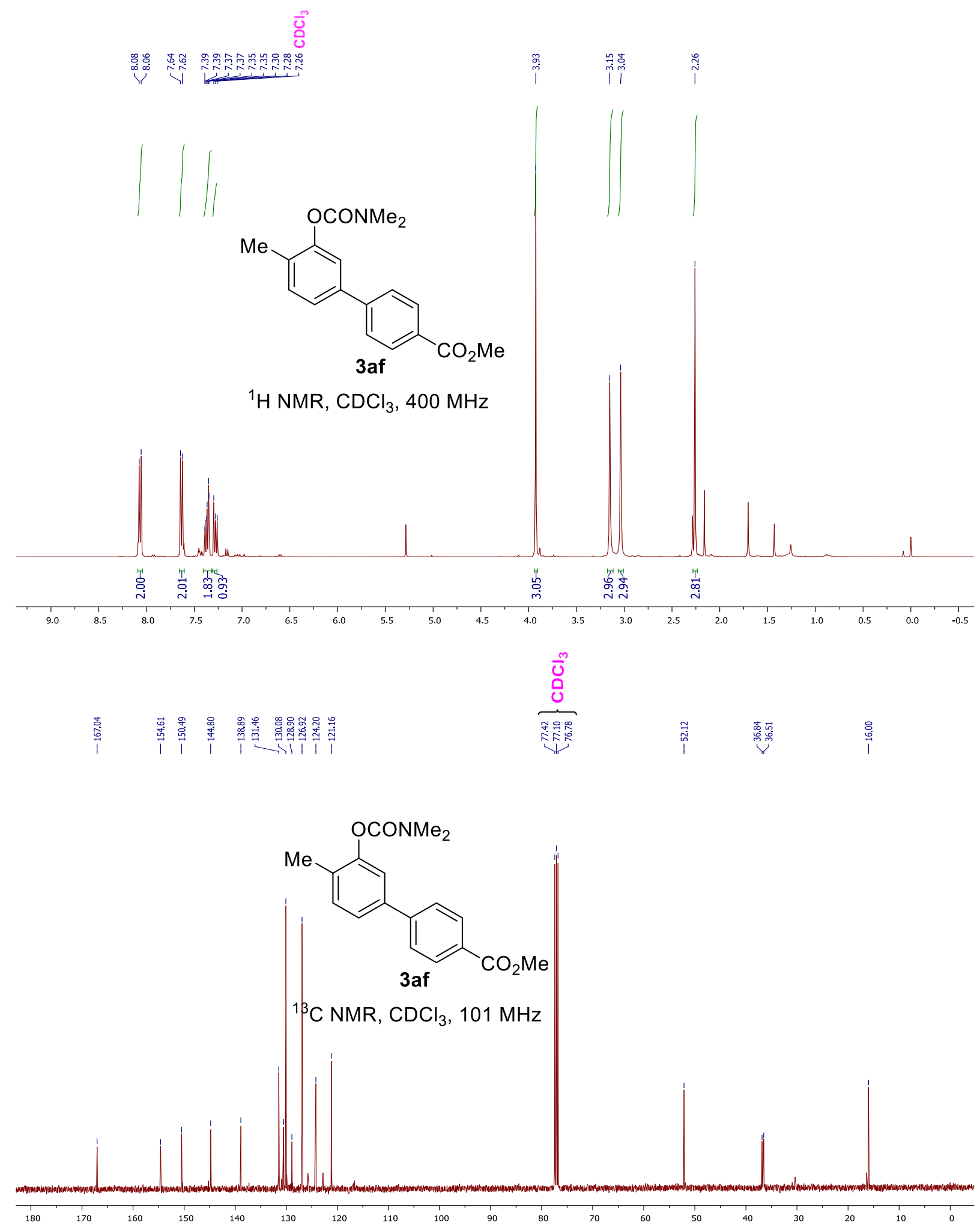


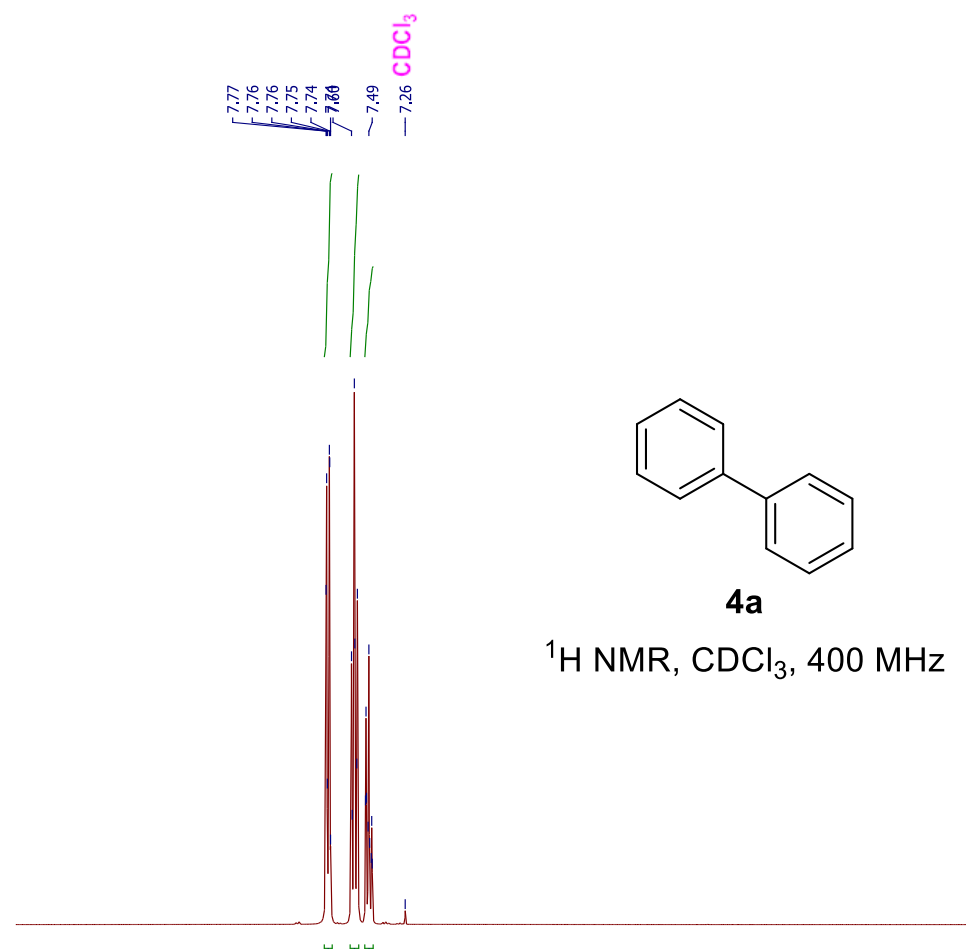

岁筞总

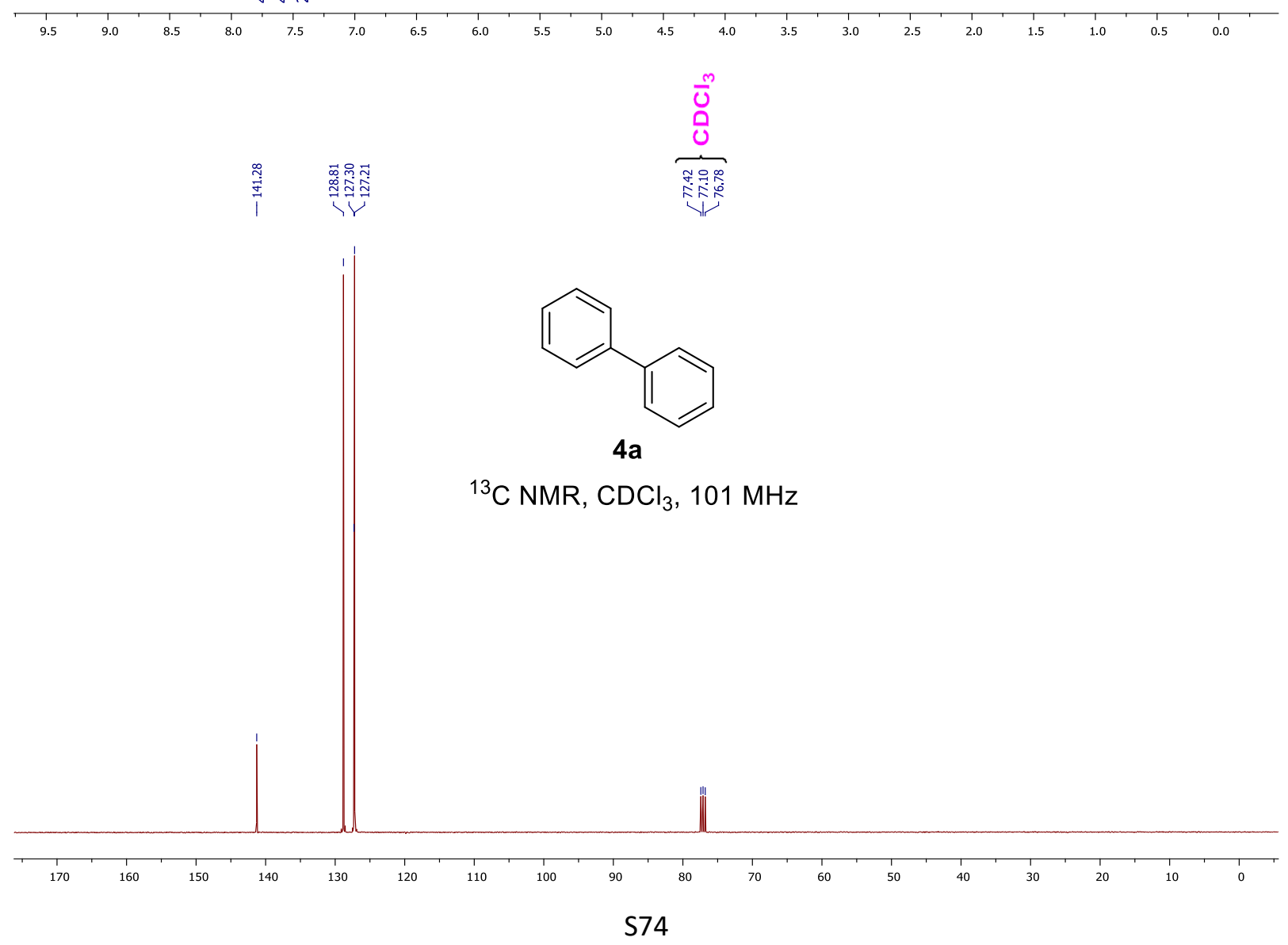




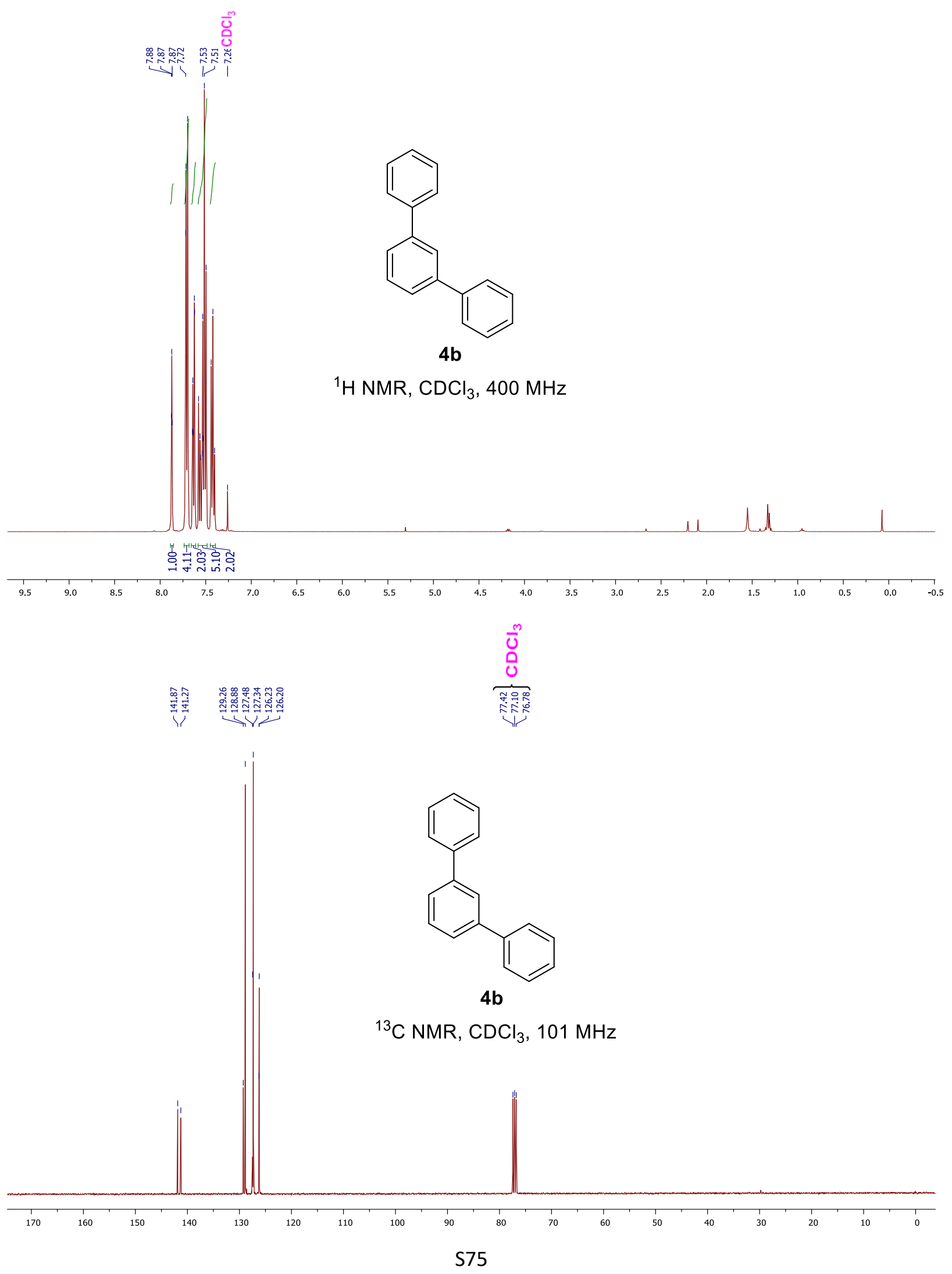

